\title{
Flavor Structure of the Nucleon Sea
}

\author{
Wen-Chen Chang ${ }^{1}$ and Jen-Chieh Peng ${ }^{2}$ \\ ${ }^{1}$ Institute of Physics, Academia Sinica, Taipei 11529, Taiwan \\ ${ }^{2}$ Department of Physics, University of Illinois at Urbana-Champaign \\ Urbana, Illinois 61801, USA
}

October 9, 2014

\begin{abstract}
We review the current status and future prospects on the subject of flavor structure of the nucleon sea. The flavor structure of the nucleon sea provides unique information on the nonperturbative aspects of strong interactions allowing stringent tests of various models on the partonic structures of the nucleons as well as lattice QCD calculations. The scope of this review covers the unpolarized, polarized, and the transverse-momentum dependent sea-quark distributions of the nucleons. While the main focus of this review is on the physics motivation and recent progress on the subject of the nucleon sea, we also discuss future prospects of addressing some outstanding issues on the flavor structure of the nucleon sea.
\end{abstract}




\section{Contents}

1 Introduction

2 Probing the Nucleon Sea 4

2.1 Deep Inelastic Scattering with Charged Lepton Beams . . . . . . . . . . . . . . . . . 44

2.2 Deep Inelastic Scattering with Neutrino or Antineutrino Beams . . . . . . . . . . . . 6

2.3 Drell-Yan Process . . . . . . . . . . . . . . . . . . . . . 7

2.4 Semi-Inclusive Deep Inelastic Scattering . . . . . . . . . . . . . . . . . . 8

3 Unpolarized Distributions of Sea Quarks $\quad 10$

3.1 Breaking of SU(2) Flavor Symmetry of Light Quarks Sea . . . . . . . . . . . . . . 11

3.2 Strange Quark Sea . . . . . . . . . . . . . . . . . . . 17

3.2.1 Breaking of SU(3) flavor symmetry of quark sea . . . . . . . . . . . . 17

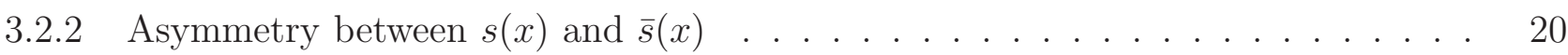

3.2.3 Strange quarks from the global analysis . . . . . . . . . . . . . . . 22

3.3 Five-quark intrinsic sea model and lattice QCD . . . . . . . . . . . . . . . 24

3.3.1 Intrinsic and extrinsic sea . . . . . . . . . . . . . . . . 24

3.3.2 Connected and disconnected sea . . . . . . . . . . . . . . 25

3.3.3 Comparison between two approaches ................. 27

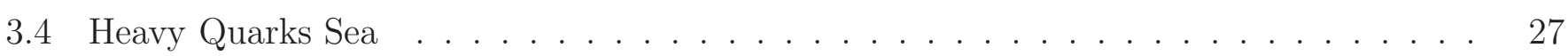

4 Polarized Distributions of Sea Quarks 29

4.1 Asymmetry Between $\Delta \bar{u}(x)$ and $\Delta \bar{d}(x) \ldots \ldots \ldots \ldots \ldots$

4.1.1 Theoretical predictions on the asymmetry between $\Delta \bar{u}(x)$ and $\Delta \bar{d}(x) \ldots$. . . . 30

4.1.2 Experimental status on the asymmetry between $\Delta \bar{u}(x)$ and $\Delta \bar{d}(x) \ldots \ldots$

4.2 Polarization of Strange Quark Sea: $\Delta s(x)$ and $\Delta s(x) \ldots \ldots \ldots$. . . . . . . 37

4.2.1 Theoretical predictions on strange quark polarizations . . . . . . . . . . 38

4.2.2 Experimental status on strange quark polarizations . . . . . . . . . . . . 39

5 Transverse Structure of the Nucleon Sea 41

5.1 Transversity Sea . . . . . . . . . . . . . . . . . . . . 41

5.2 Sivers Sea ................................ 44

5.3 Boer-Mulders Sea . . . . . . . . . . . . . . . . . . . . 45

6 Experimental Perspectives $\quad 48$

6.1 Fixed-Target Experiments . . . . . . . . . . . . . . . . . . . . 49

6.2 Collider Experiments .......................... 50

\begin{tabular}{lll}
7 & Summary & 51 \\
\hline
\end{tabular} 


\section{Introduction}

The observation of the Bjorken- $x$ scaling behavior in deep inelastic scattering (DIS) revealed quarks as the point-like constituents of the nucleons [1. The existence of sea quarks was further suggested from the DIS data, showing a prominent rise of the partonic density at small $x$. The discovery of the Drell-Yan process in hadron-hadron collision, which involves the annihilation of a quark-antiquark pair, provided a further experimental evidence for the existence of sea quarks in the nucleons.

Unlike the situation in atomic systems, where the effects of particle-antiparticle pairs are relatively minor, the quark-antiquark pairs have important roles in describing the internal structures of nucleons and other hadrons. The large magnitude of the coupling constant $\alpha_{s}$ in QCD implies that quarkantiquark pairs are readily produced. The sea quarks, like the valence quarks and gluons, form an integral part of the nucleon's structure. After several decades of extensive experimental and theoretical studies, the valence quark distributions of the nucleons are quite well known. In contrast, some important aspects of the sea quarks, such as their flavor and spin dependence, are just beginning to be explored.

In this article, we review the current status and future prospects for our understanding of the flavor and spin structures of the sea quark contents in the nucleons. While the valence quarks in the nucleons are restricted to the up and down flavors, the flavor structure of the sea quarks is potentially richer, extending beyond the light quarks. For the light quark flavor dependence, a major surprise was found when DIS and Drell-Yan experiments showed that the $\bar{u}$ and $\bar{d}$ have very different $x$ distributions. As discussed later, this unexpected finding has generated much interest and has provided new insights on the origins of the nucleons sea. At the Large Hadron Collider (LHC) or the future Electron Ion Collider (EIC), the role of sea quarks becomes more important, since the densities of the sea quarks, especially for the heavy flavors, are expected to rise at the large $Q^{2}$ and small $x$ regions explored in these high energy colliders. This offers the opportunity to study the flavor structures of the light quark sea $(\bar{u}, \bar{d})$ at new kinematic regions, as well as the heavy quark sea $(s, c, b)$ of which much less is known.

Another intriguing aspect of sea quarks is their spin structure. Following the discovery of the "spin puzzle" in the 1980s, the decomposition of the proton's spin in terms of the spin and orbital motion of the constituent quarks, antiquarks, and gluons continued to be a major unresolved issue in high energy spin physics. The flavor and $x$ dependence of the antiquark helicity distributions could offer important insights for solving this puzzle. Moreover, many theoretical models capable of explaining the difference between the unpolarized $\bar{u}$ and $\bar{d}$ momentum distributions also have distinct predictions for the flavor dependence of the polarized sea. New information on the flavor structure of the sea quark polarization would provide a stringent test for these models and would further advance our understanding on the origin of the $\bar{d} / \bar{u}$ flavor asymmetry.

During the recent decades, significant progress has been made in delineating the roles of the transverse momentum and transverse spin of the quarks in nucleon structure. The properties of some novel parton distributions involving these transverse degrees of freedom have been investigated theoretically and first data are becoming available from the semi-inclusive DIS measurements. The possibility to measure the transverse spin and transverse momentum dependent sea-quark distributions will offer another interesting view on the nature of the nucleon sea.

In this review article, we discuss the recent progress and future prospects for understanding the flavor and spin structure of the nucleon sea. This article is organized as follows. In Section 2, we present a brief overview of the relevant experimental tools in exploring the sea quarks. In Section 3, we discuss several intriguing aspects of the flavor structure of unpolarized nucleon sea. This is followed by discussions on the helicity distributions of the nucleon sea in Section 4. We then briefly review the topics of transverse spin or transverse-momentum dependent sea quark structures, which is just beginning to be explored. In Section 5, we highlight future prospects for advancing our current knowledge on various outstanding issues in the subject of nucleon sea, followed by summary and conclusion in Section 6. 


\section{Probing the Nucleon Sea}

Our knowledge about the partonic structure of the nucleon [2, 3, 4, 5, 6, 7, 8] mainly comes from the deep inelastic scattering (DIS) using either charged lepton or neutrino beams [9]. The DIS is in general not effective in separating the sea quarks from the valence quarks. However, by detecting other particles (hadrons or charged leptons) in coincidence with the scattered leptons, the semi-inclusive DIS (SIDIS) process is sensitive to the flavor of the struck quark or antiquark. Complementary information could be obtained from the Drell-Yan process [10] where the quark and antiquark from two interacting hadrons annihilate into a virtual photon, $Z$, or $W$ bosons [11, 12]. This process yields particularly important information on the sea quark content of the nucleons.

Below we briefly introduce the kinematics of DIS, Drell-Yan and SIDIS processes, following the formulation of Refs. [13, 14, 15] and provide the leading order expressions of the production cross sections. We end this Section with a table listing the various reactions and the associated subprocesses which are relevant for probing sea quarks in the nucleon.

\subsection{Deep Inelastic Scattering with Charged Lepton Beams}

Figure 1 illustrates the deep inelastic scattering process of charged lepton beam off a nuclear $l N \rightarrow l X$ or $l N \rightarrow \nu X$ where $l$ denotes the charged leptons, $\nu$ the neutrino, $N$ the nucleon target and $X$ the undetected hadronic final state. At leading order, the lepton couples to the nucleon either through a virtual photon $\gamma^{*}, Z$ or $W$ boson. In the past these processes were studied in the fixed-target experiments except the collider experiments at HERA.

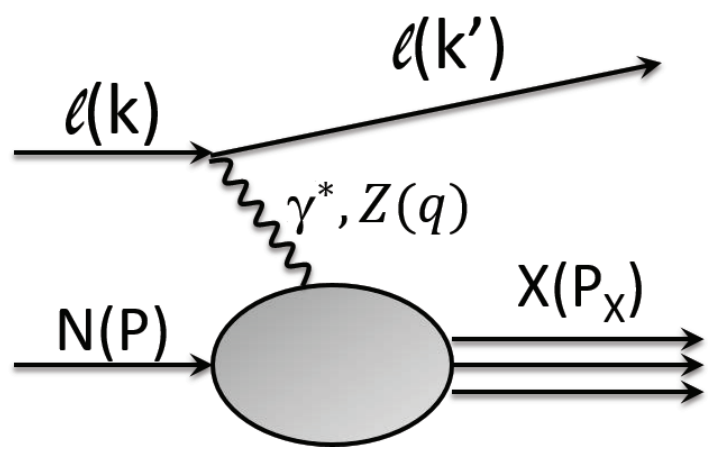

(a)

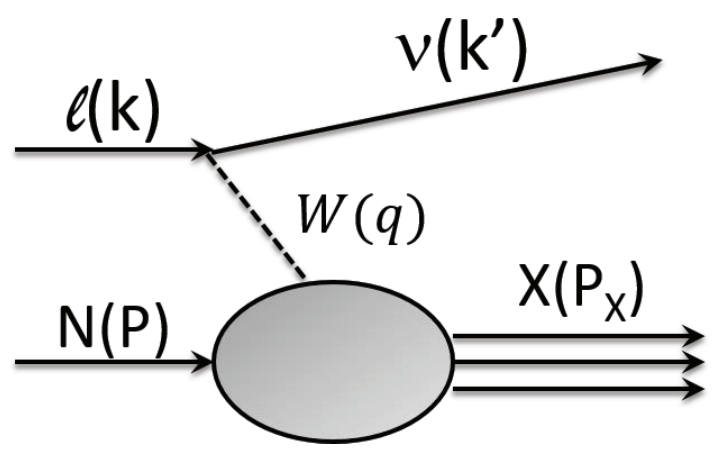

(b)

Figure 1: Inclusive lepton-hadron scattering via (a) $\gamma^{*}, Z$ (b) $W$ exchange.

The four-momentum vectors of the incoming and outgoing leptons are denoted as $k$ and $k^{\prime}$ respectively, and $P$ is that of the nucleon. The momentum of the exchanged boson is $q=k-k^{\prime}$, and the momentum of the hadronic final state system $X$ is $P_{X}=P+q$. The virtuality of the exchanged boson in DIS is spacelike, i.e. $q^{2}<0$.

Below we list several Lorentz invariants and kinematic variables useful for the description of the kinematics of the process:

- $s=(k+P)^{2}$ : the center of mass energy squared for the $l N$ system.

- $Q^{2}=-q^{2} \equiv-\left(k-k^{\prime}\right)^{2}$ : the magnitude of the invariant mass squared of the exchanged boson.

- $\nu=(P \cdot q) / M_{N}=E_{k}-E_{k^{\prime}}$ : the energy transferred from the incident lepton to the target nucleon in the target rest frame. 
- $x=Q^{2} /(2 P \cdot q)=Q^{2} /\left(2 M_{N} \nu\right)$ : the dimensionless Bjorken scaling variable, which represents the nucleon momentum fraction of the struck quark in the quark-parton.

- $W^{2}=(P+q)^{2}$ : the invariant mass squared of the hadronic system $X$ in the final state.

- $y=(P \cdot q) /(P \cdot k)=\nu / E_{k}$ : the ratio of the transferred energy over the total lepton energy in the target nucleon rest frame.

The $x, Q^{2}$ and $y$ are related to $s$ by the relation of $Q^{2}=s x y$ in the massless limit and thus they are not completely independent variables. Usually the differential cross sections are given in two of them: $x, Q^{2}$ or $x, y$.

For charged lepton-nucleon scattering, mediated by the neutral current (NC), i.e. the exchange of $\gamma^{*}$ or $Z$, the differential cross section may be written in terms of a leptonic and a hadronic tensor and their coupling via the exchanged boson. The hadronic tensor is not known from first principle and represented by three structure functions, $F_{2}, F_{L}$ and $x F_{3}$. The differential cross section is expressed as [13]

$$
\frac{d^{2} \sigma^{N C}\left(l^{ \pm} N\right)}{d x d Q^{2}}=\frac{2 \pi \alpha^{2}}{x Q^{4}}\left[Y_{+} F_{2}^{N C}\left(x, Q^{2}\right)-y^{2} F_{L}^{N C}\left(x, Q^{2}\right) \mp Y_{-} x F_{3}^{N C}\left(x, Q^{2}\right)\right],
$$

where $\alpha$ is the fine structure constant. The factor $Y_{ \pm} \equiv 1 \pm(1-y)^{2}$ is due to the helicity dependence of the electroweak interaction.

Provided that $Q^{2} \gg 4 M_{N}^{2} x^{2}$ and $Q^{2}$ is well below that of the $Z$ mass squared $\left(M_{Z}^{2}\right)$, the parity violating structure function $x F_{3}^{N C}$ is negligible. Furthermore there is no longitudinal absorption cross section for $\gamma^{*}$ scattering on quarks, i.e. $F_{L}=0$, because of the spin-1/2 nature of quarks. Therefore the differential cross section of charged lepton-nucleon scattering comes only from the first term in Eq. 1,

$$
\frac{d^{2} \sigma^{N C}\left(l^{ \pm} N\right)}{d x d Q^{2}}=\frac{2 \pi \alpha^{2}}{x Q^{4}} Y_{+} F_{2}^{N C}\left(x, Q^{2}\right) .
$$

In the parton model, only charged partons of the hadron, the quarks and antiquarks, couple to the electroweak currents at leading order. The structure function $F_{2}^{N C}\left(x, Q^{2}\right)$ could be expressed in terms of the parton density of the quark $q_{i}$ and antiquark $\bar{q}_{i}$ in the nucleon and the quark charge squared $e_{i}^{2}$ as follows:

$$
F_{2}^{N C}\left(x, Q^{2}\right)=\sum_{i=1}^{n_{f}} e_{i}^{2}\left[x q_{i}\left(x, Q^{2}\right)+x \bar{q}_{i}\left(x, Q^{2}\right)\right],
$$

where index $i$ refers to the quark flavor. Therefore the lepton-nucleon scattering process has been the main experimental tool for measuring the quark distribution functions of nucleons and investigating their $Q^{2}$ dependence.

When $Q^{2}$ becomes comparable to $M_{Z}^{2}$, the $Z$ exchange and the effect of $\gamma^{*}-Z$ interference have to be taken into account and the parity violating structure function $x F_{3}$ can no longer be neglected. The complete expression is referred to, for example, Ref. [13].

As for the charged-current (CC) where $W^{ \pm}$is exchanged and the final state lepton is a (anti)neutrino, the charged lepton-nucleon differential cross sections are expressed as 13

$$
\frac{d^{2} \sigma^{C C}\left(l^{ \pm} N\right)}{d x d Q^{2}}=\frac{G_{F}^{2}}{4 \pi x}\left[\frac{M_{W}^{2}}{\left(Q^{2}+M_{W}^{2}\right)}\right]^{2}\left[Y_{+} F_{2}^{C C}\left(x, Q^{2}\right)-y^{2} F_{L}^{C C}\left(x, Q^{2}\right) \mp Y_{-} x F_{3}^{C C}\left(x, Q^{2}\right)\right]
$$

and the Fermi coupling constant $G_{F}$ is

$$
G_{F}=\frac{\pi \alpha}{\sqrt{2} \sin ^{2} \theta_{W} M_{W}^{2}},
$$


where $\theta_{W}$ is the Weinberg angle and $M_{W}$ the mass of $W$ boson.

In the parton model, $F_{L}$ vanishes in LO pQCD. Neglecting the contribution from top $(t)$ and bottom $(b)$ quarks, the structure functions $F_{2}^{C C}$ and $x F_{3}^{C C}$ are expressed by the sums and differences of quarks and antiquarks densities as

$$
\begin{aligned}
F_{2}^{C C}\left(l^{-} N\right) & =2 x(u+c+\bar{d}+\bar{s}) \\
x F_{3}^{C C}\left(l^{-} N\right) & =2 x(u+c-\bar{d}-\bar{s})
\end{aligned}
$$

for the lepton beam of left-handed polarization, and

$$
\begin{aligned}
F_{2}^{C C}\left(l^{+} N\right) & =2 x(\bar{u}+\bar{c}+d+s) \\
x F_{3}^{C C}\left(l^{+} N\right) & =2 x(d+s-\bar{u}-\bar{c})
\end{aligned}
$$

for that of right-handed polarization. Hence the differential cross section of lepton scattering is given by 13

$$
\frac{d^{2} \sigma^{C C}\left(l^{-} N\right)}{d x d Q^{2}}=(1-P) \frac{G_{F}^{2}}{2 \pi x}\left[\frac{M_{W}^{2}}{\left(Q^{2}+M_{W}^{2}\right)}\right]^{2} \sum_{i=1}^{n_{f}}\left[x q_{i}\left(x, Q^{2}\right)+(1-y)^{2} x \bar{q}_{i}\left(x, Q^{2}\right)\right]
$$

whereas that of antilepton scattering,

$$
\frac{d^{2} \sigma^{C C}\left(l^{+} N\right)}{d x d Q^{2}}=(1+P) \frac{G_{F}^{2}}{2 \pi x}\left[\frac{M_{W}^{2}}{\left(Q^{2}+M_{W}^{2}\right)}\right]^{2} \sum_{i=1}^{n_{f}}\left[(1-y)^{2} x q_{i}\left(x, Q^{2}\right)+x \bar{q}_{i}\left(x, Q^{2}\right)\right]
$$

where the summation goes over only the quarks or antiquarks relevant for the charge of the exchanged current and $P$ is the polarization degree of the lepton beam, $P=\left(N_{R}-N_{L}\right) /\left(N_{R}+N_{L}\right)$.

\subsection{Deep Inelastic Scattering with Neutrino or Antineutrino Beams}

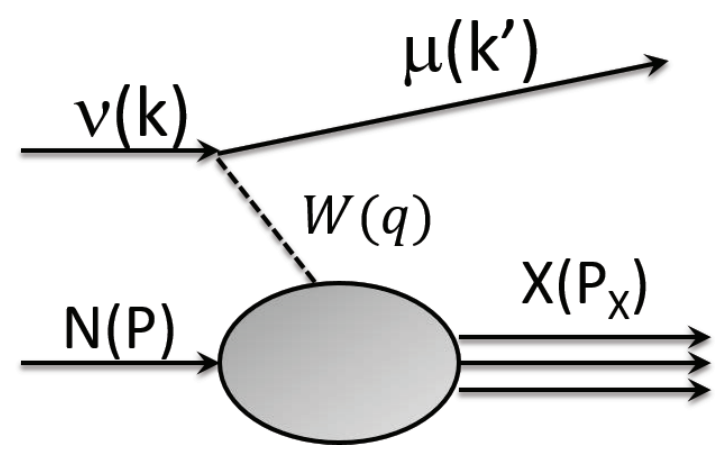

Figure 2: Inclusive neutrino scattering via $W$ exchange.

The charged-current interactions were most extensively studied in the neutrino and antineutrino inelastic scattering processes, where

$$
\nu(\bar{\nu}) N \rightarrow \mu^{-}\left(\mu^{+}\right) X
$$

at $Q^{2} \ll M_{W}^{2}$, as illustrated in Fig. 2, and the cross sections are expressed as

$$
\frac{d^{2} \sigma^{C C}(\nu, \bar{\nu})}{d x d Q^{2}}=\frac{(1 \pm P)}{2} \frac{G_{F}^{2}}{4 \pi x}\left[Y_{+} F_{2}^{C C}\left(x, Q^{2}\right)-y^{2} F_{L}^{C C}\left(x, Q^{2}\right) \pm Y_{-} x F_{3}^{C C}\left(x, Q^{2}\right)\right] .
$$

Since the polarizations of the (anti)neutrino probes are intrinsically (right)left-handed, both polarization factors of the beam $(1 \pm P)$ become 2 now. 
In LO pQCD the differential cross sections of neutrino scattering expressed by the nucleon parton densities are

$$
\frac{d^{2} \sigma(\nu)}{d x d Q^{2}}=\frac{G_{F}^{2}}{\pi x} \sum_{i=1}^{n_{f}}\left[x q_{i}\left(x, Q^{2}\right)+(1-y)^{2} x \bar{q}_{i}\left(x, Q^{2}\right)\right]
$$

and those for antineutrino scattering,

$$
\frac{d^{2} \sigma(\bar{\nu})}{d x d Q^{2}}=\frac{G_{F}^{2}}{\pi x} \sum_{i=1}^{n_{f}}\left[(1-y)^{2} x q_{i}\left(x, Q^{2}\right)+x \bar{q}_{i}\left(x, Q^{2}\right)\right]
$$

where the sums go through only the appropriate quarks or antiquarks for the charge of the current. Because of the different coupling strength of the exchanged $W$ boson with the quarks and antiquarks, the combination of the data from neutrino and antineutrino DIS experiments are used to extract the individual quark and antiquark densities. The other combination of data from proton and a heavy isoscalar target could lead to flavor separation of parton densities.

\subsection{Drell-Yan Process}

As illustrated in Fig. 3, the Drell-Yan (DY) process is the production of a lepton pair with large invariant mass $\left(l^{+} l^{-}\right.$and $\left.l \nu\right)$ in the collisions of two hadrons. Different from DIS, the virtual boson formed in DY is timelike, i.e. $q^{2}=Q^{2}>0$. The square of the center of mass energy for two colliding hadrons is $s \equiv\left(P_{A}+P_{B}\right)^{2}$. In the parton model, the intermediate bosons $\left(\gamma^{*}, Z, W\right)$ are produced by the annihilation of quark-antiquark pair, which then couple to the lepton pair through electromagnetic or weak interactions. In general the DY process yields information complementary to what is revealed in DIS and is particularly useful in probing the distributions of antiquarks.

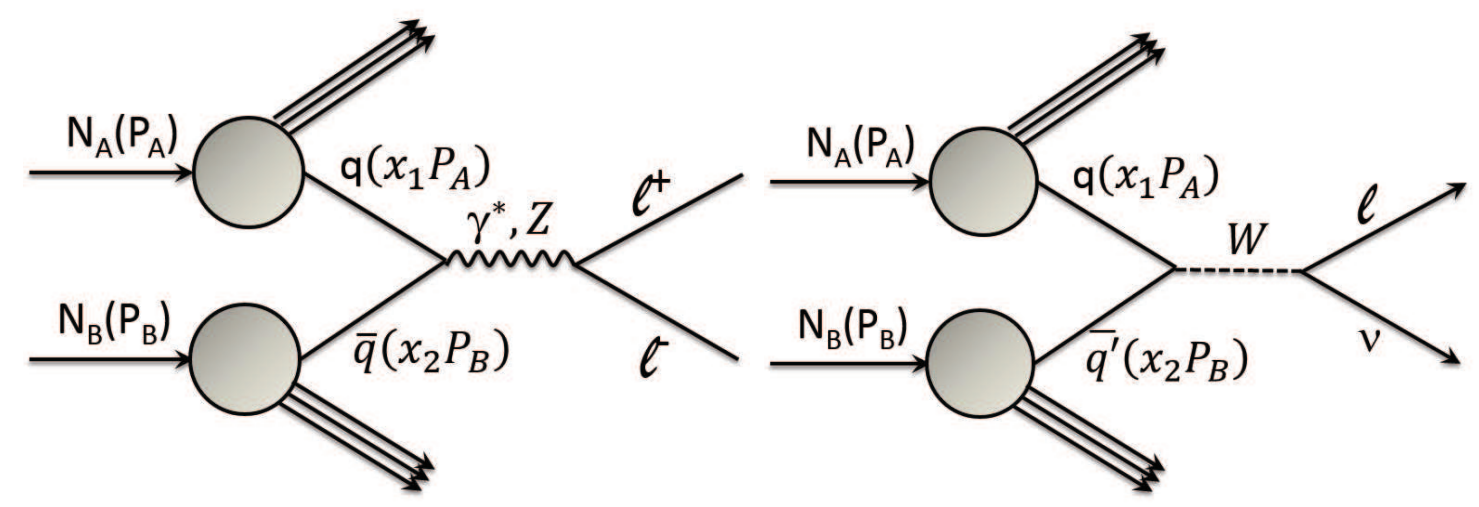

(a)

(b)

Figure 3: Drell-Yan process for (a) $l^{+} l^{-}$and (b) $l \nu$ production in the collisions of two nucleons.

The inclusive DY cross section of collisions of hadron $A$ and $B$ is expressed as [14]

$$
\begin{aligned}
\frac{d \sigma^{V}}{d Q^{2}} & =\sigma_{0}^{V} W_{A B}^{V}(\tau) \\
W_{A B}^{V}(\tau) & =\int_{0}^{1} d x_{1} \int_{0}^{1} d x_{2} \delta\left(\tau-x_{1} x_{2}\right) D_{A B}^{V}
\end{aligned}
$$

with $V=\gamma^{*}, Z$ or $W$, and $\tau=Q^{2} / s$ is the DY scaling variable. The factor $\sigma_{0}^{V}$ contains the full dimensions of $d \sigma^{V} / d Q^{2}$ and the dimensionless function $W_{A B}^{V}$ is the convoluted integral over the product of the parton distributions in the projectile and target hadrons denoted as $D_{A B}^{V}$. The $x_{1}$ and $x_{2}$ are the momentum fractions of interacting partons in the hadron $A$ and $B$ respectively. 
For the production of virtual photon $\gamma^{*}$, we have

$$
\begin{aligned}
\sigma_{0}^{\gamma^{*}} & =\frac{4 \pi \alpha^{2}}{3 N_{c} Q^{2} s} \\
D_{A B}^{\gamma^{*}}\left(x_{1}, x_{2}\right) & =\sum_{i=1}^{n_{f}} e_{i}^{2}\left\{q_{i}^{A}\left(x_{1}\right) \bar{q}_{i}^{B}\left(x_{2}\right)+(A \leftrightarrow B)\right\}
\end{aligned}
$$

where $N_{c}$ is the number of color charge.

In the case of $Z$ boson production, the $\sigma_{0}^{V}$ and $D_{A B}^{V}$ become

$$
\begin{aligned}
\sigma_{0}^{Z} & =\frac{\pi \alpha^{2}}{192 N_{c} \sin ^{4} \theta_{W} \cos ^{4} \theta_{W}} \frac{q^{2}}{s} \frac{1+\left(1-4 \sin ^{4} \theta_{W}\right)^{2}}{\left(Q^{2}-M_{Z}^{2}\right)^{2}+M_{Z}^{2} \Gamma_{Z}^{2}} \\
D_{A B}^{Z}\left(x_{1}, x_{2}\right) & =\sum_{i=1}^{n_{f}}\left[1+\left(1-4\left|e_{i}\right| \sin ^{2} \theta_{W}\right)^{2}\right]\left\{q_{i}^{A}\left(x_{1}\right) \bar{q}_{i}{ }^{B}\left(x_{2}\right)+(A \leftrightarrow B)\right\}
\end{aligned}
$$

where $\theta_{W}$ is the Weinberg angle and $M_{z}$ and $\Gamma_{z}$ are the mass and total width of the $Z$ boson.

Similarly, the corresponding results for the case of $V=W^{-}$are

$$
\begin{aligned}
\sigma_{0}^{W^{-}} & =\frac{\pi \alpha^{2}}{12 N_{c} \sin ^{4} \theta_{W}} \frac{q^{2}}{s} \frac{1}{\left(Q^{2}-M_{W}^{2}\right)^{2}+M_{W}^{2} \Gamma_{W}^{2}} \\
D_{A B}^{W^{-}}\left(x_{1}, x_{2}\right) & =\cos ^{2} \theta_{C}\left[d^{A}\left(x_{1}\right) \bar{u}^{B}\left(x_{2}\right)+s^{A}\left(x_{1}\right) \bar{c}^{B}\left(x_{2}\right)\right] \\
& +\sin ^{2} \theta_{C}\left[s^{A}\left(x_{1}\right) \bar{u}^{B}\left(x_{2}\right)+u^{A}\left(x_{1}\right) \bar{c}^{B}\left(x_{2}\right)\right] \\
& +\{A \leftrightarrow B\}
\end{aligned}
$$

where $\theta_{C}$ is the Cabibbo mixing angle and $M_{W}$ and $\Gamma_{W}$ are the mass and total width of the $W$ boson. The results for $V=W^{+}$could be obtained by charge conjugation operation on the parton densities.

\subsection{Semi-Inclusive Deep Inelastic Scattering}

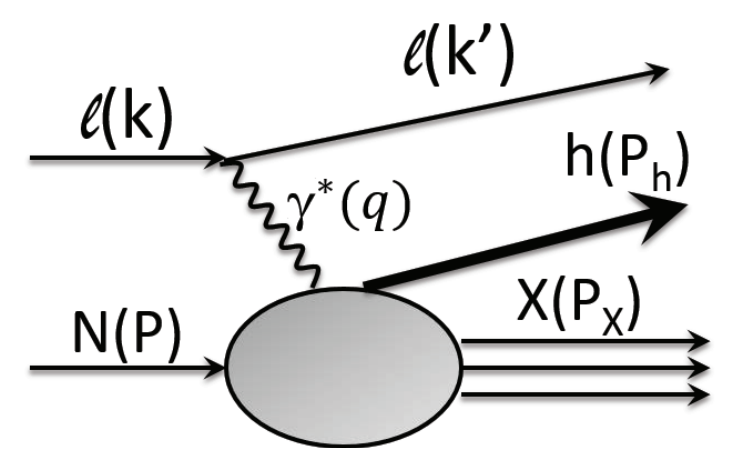

(a)

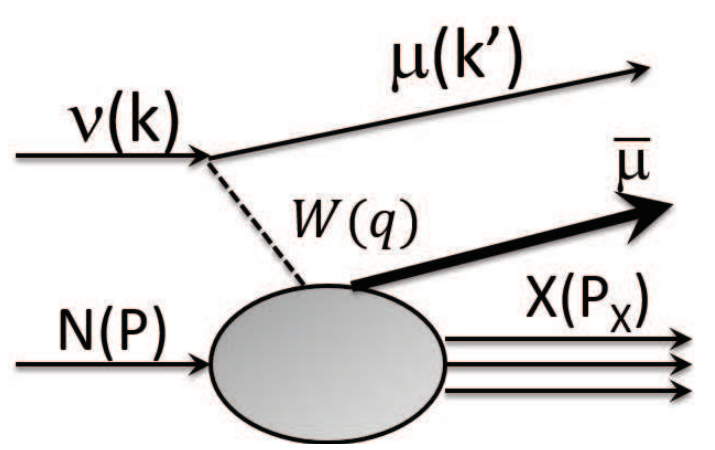

(b)

Figure 4: Semi-inclusive deep inelastic scattering via (a) $\gamma^{*}$ (b) $W$ exchange.

When certain hadrons or leptons produced in the DIS are detected together with the scattered lepton, the process is then called "semi-inclusive DIS" (SIDIS). As illustrated in Fig. 4(a) for the case of SIDIS production of hadron $h$, the leading order cross section is proportional to the combination of nucleon parton density $q\left(x, Q^{2}\right)$ and the fragmentation function $D_{q}^{h}\left(z, Q^{2}\right)$ expressed as:

$$
\frac{d \sigma^{h}}{d x d y d z}(x, y, z)=\frac{2 \pi \alpha^{2}}{y Q^{2}} Y_{+} \sum_{q, \bar{q}} e_{q}^{2} q\left(x, Q^{2}\right) D_{q}^{h}\left(z, Q^{2}\right)
$$


where $Y_{+}=1+(1-y)^{2}$ and $D_{q}^{h}\left(z, Q^{2}\right)$ is the probability of a quark $q$ fragmenting into a hadron $h$ of four-momentum $P_{h}$ with an energy fraction $z\left(=P_{h} \cdot P / q \cdot P\right)$. The measured hadron multiplicities of hadron $h$ after being normalized to the number of DIS events could be expressed as:

$$
\frac{d N^{h}(x, z)}{d N^{D I S}}=\frac{\sum_{q, \bar{q}} e_{q}^{2} q\left(x, Q^{2}\right) D_{q}^{h}\left(z, Q^{2}\right)}{\sum_{q, \bar{q}} e_{q}^{2} q\left(x, Q^{2}\right)} .
$$

The neutrino and antineutrino scattering with $W$ exchange could lead to the production of charmed quarks and such process can be viewed as an example of SIDIS, illustrated in Fig. 4(b). It has been used as an experimental approach to differentiate the nucleon strange quark distributions, $s(x)$ and $\bar{s}(x)$. The signature for the production of charmed quarks in neutrino- and antineutrino-nucleon scattering is the presence of a $\mu^{+} \mu^{-}$pair in the final state.

In the case of neutrino scattering, the underlying process is a neutrino interacting with an $s$ or $d$ quark, producing a charm quark which fragments into a charmed hadron, i.e. $\nu_{\mu} \mathrm{N} \rightarrow \mu^{-} c \mathrm{X} ; c \rightarrow D^{+} \mathrm{X}$, and followed by the semi-leptonic decay of charmed hadron which produces a second muon of opposite sign, $D^{+} \rightarrow \mu^{+} \nu_{\mu} X$. Likewise the process with an incident antineutrino involves interaction with an $\bar{s}$ or $\bar{d}$ antiquark, again leading to oppositely-signed muons in the final state.

The differential cross section for dimuon production is expressed generally as [15]

$$
\frac{d^{3} \sigma\left(\nu_{\mu} N \rightarrow \mu^{-} \mu^{+} X\right)}{d x d y d z}=\frac{d^{2} \sigma\left(\nu_{\mu} N \rightarrow \mu^{-} c X\right)}{d x d y} D_{c}^{h}(z) B_{c}\left(c \rightarrow \mu^{+} X\right)
$$

where $x$ is the momentum fraction of the struck quark, the function $D_{c}^{h}(z)$ the hadronization of charmed quarks and $B_{c}$ the weighted average of the semi-leptonic branching ratios of the charmed hadrons produced in neutrino interactions.

The leading order differential cross section for an isoscalar target, neglecting target mass effects, is given by:

$$
\begin{aligned}
\frac{d^{2} \sigma\left(\nu_{\mu} N \rightarrow \mu^{-} c X\right)}{d x d y}= & \frac{G_{F}^{2} M_{N} E_{\nu}}{\pi\left(1+Q^{2} / M_{W}^{2}\right)^{2}}\left\{\left[x u\left(x, Q^{2}\right)+x d\left(x, Q^{2}\right)\right]\left|V_{c d}\right|^{2}\right. \\
& \left.+2 x s\left(x, Q^{2}\right)\left|V_{c s}\right|^{2}\right\}\left(1-\frac{m_{c}^{2}}{2 M_{N} E_{\nu} x}\right)
\end{aligned}
$$

where $x u\left(x, Q^{2}\right), x d\left(x, Q^{2}\right)$ and $x s\left(x, Q^{2}\right)$ represent the momentum distributions of the $u, d$ and $s$ quarks within the proton (the corresponding $\bar{\nu}_{\mu}$ process has the quarks replaced by their antiquark partners) respectively and $\left|V_{c d}\right|$ and $\left|V_{c s}\right|$ are the CKM matrix elements. The difference between neutrino and antineutrino induced dimuon differential cross section is expressed as:

$$
\begin{aligned}
\frac{d^{2} \sigma\left(\nu_{\mu} N \rightarrow \mu^{-} c X\right)}{d x d y}-\frac{d^{2} \sigma\left(\bar{\nu}_{\mu} N \rightarrow \mu^{+} \bar{c} X\right)}{d x d y}= & \frac{G_{F}^{2} M_{N} E_{\nu}}{\pi\left(1+Q^{2} / M_{W}^{2}\right)^{2}}\left\{\left[x u_{v}\left(x, Q^{2}\right)+x d_{v}\left(x, Q^{2}\right)\right]\left|V_{c d}\right|^{2}\right. \\
& \left.+2 x\left(s\left(x, Q^{2}\right)-\bar{s}\left(x, Q^{2}\right)\right)\left|V_{c s}\right|^{2}\right\}\left(1-\frac{m_{c}^{2}}{2 M_{N} E_{\nu} x}\right)(26)
\end{aligned}
$$

The contribution of valence quarks in Eq. 26 is suppressed relative to the strange contribution because of the large difference in the coefficients: $\left|V_{c d}\right|^{2} \sim 0.05$ and $\left|V_{c s}\right|^{2} \sim 0.9$. Therefore the difference of cross sections in two reactions is sensitive to the strange distribution asymmetry $x\left(s\left(x, Q^{2}\right)-\bar{s}\left(x, Q^{2}\right)\right)$.

We conclude this section by summarizing the experimental reactions and the associated subprocesses which are sensitive to the nucleon sea quarks in Table 1. 


\begin{tabular}{lll}
\hline \hline Process & Subprocess & Partons Probed \\
\hline$l N \rightarrow l X$ & $\left\{\gamma^{*}, Z\right\} q, \bar{q} \rightarrow q, \bar{q}$ & $q, \bar{q}$ \\
$l^{-} p \rightarrow \nu X$ & $W^{-}\{u, c, \bar{d}, \bar{s}\} \rightarrow\{d, s, \bar{u}, \bar{c}\}$ & $u, c, \bar{d}, \bar{s}$ \\
$l^{+} p \rightarrow \bar{\nu} X$ & $W^{+}\{d, s, \bar{u}, \bar{c}\} \rightarrow\{u, c, \bar{d}, \bar{s}\}$ & $d, s, \bar{u}, \bar{c}$ \\
$l N \rightarrow l\{\pi, K\} X$ & $\left\{\gamma^{*}, Z\right\} q, \bar{q} \rightarrow\{\pi, K\}$ & $u, d, s, \bar{u}, \bar{d}, \bar{s}$ \\
\hline$\nu p \rightarrow \mu^{-} X$ & $W^{-}\{u, c, d, \bar{s}\} \rightarrow\{d, s, \bar{u}, \bar{c}\}$ & $u, c, d, \bar{s}$ \\
$\bar{\nu} p \rightarrow \mu^{+} X$ & $W^{+}\{d, s, \bar{u}, \bar{c}\} \rightarrow\{u, c, \bar{d}, \bar{s}\}$ & $d, s, \bar{u}, \bar{c}$ \\
$\nu N \rightarrow \mu^{-} \mu^{+} X$ & $W^{+} s \rightarrow c$ & $s$ \\
$\bar{\nu} N \rightarrow \mu^{+} \mu^{-} X$ & $W^{-} \bar{s} \rightarrow \bar{c}$ & $\bar{s}$ \\
\hline$N N \rightarrow \mu^{+} \mu^{-} X$ & $q \bar{q} \rightarrow\left\{\gamma^{*}, Z\right\}$ & $q, \bar{q}$ \\
$N N \rightarrow W^{-} X$ & $q \bar{q}^{\prime} \rightarrow W^{-}$ & $d, s, \bar{u}, \bar{c}$ \\
$N N \rightarrow W^{+} X$ & $q \bar{q}^{\prime} \rightarrow W^{+}$ & $u, c, \bar{d}, \bar{s}$ \\
\hline \hline
\end{tabular}

Table 1: The experimental reactions and the subprocesses sensitive to the parton density of nucleon sea quarks.

\section{Unpolarized Distributions of Sea Quarks}

A simple picture of the quark sea from the perturbative quark-antiquark pair production by gluons would lead to the following expectations:

- The sea is composed of equal amount of $\bar{u}$ and $\bar{d}$ because gluon is flavor-blind and $u$ and $d$ quarks roughly have equal mass.

- Assuming SU(3) symmetry, the $x$ distribution of $\bar{u}, \bar{d}, \bar{s}$ (or $s$ ) will be identical.

- The sea quarks would be distributed mainly at the small- $x$ region where gluons are abundant.

Figure 5 shows the $x$ distributions of unpolarized parton density for valence quarks $u_{v}, d_{v}$ and sea quarks $\bar{u}, \bar{d}, \bar{s}$ from next-to-next-to-leading order (NNLO) global analysis, CT10 [16], MSTW2008 [17] and NNPDF2.3 [18, at $Q^{2}=4 \mathrm{GeV}^{2}$. Obviously the naive expectations of $\mathrm{SU}(2)$ and $\mathrm{SU}(3)$ flavor symmetry of sea quarks are not observed in the experimental data and there exists interesting flavor structure. In this Section, we review the recent experimental and theoretical progress in understanding this flavor structure of the unpolarized nucleon sea of $u, d, s$ and the valence-like charm sea.
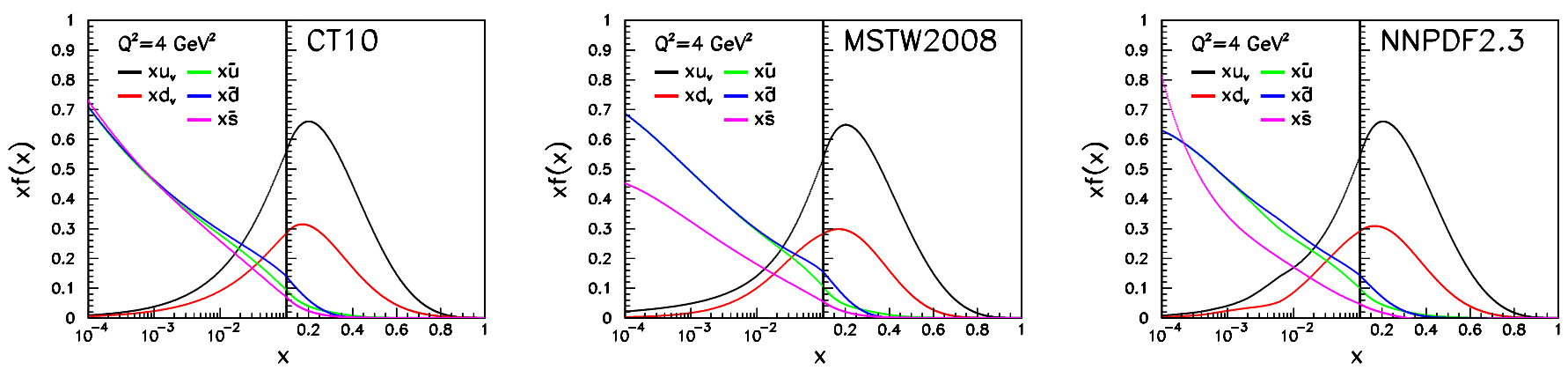

Figure 5: Unpolarized parton distribution $x f\left(x, Q^{2}\right)$ of valence quarks $u_{v}, d_{v}$ and sea quarks $\bar{u}, \bar{d}, \bar{s}$ from CT10 [16], MSTW2008 [17] and NNPDF2.3 [18] PDFs at $Q^{2}=4 \mathrm{GeV}^{2}$. 


\subsection{Breaking of SU(2) Flavor Symmetry of Light Quarks Sea}

The first hint of flavor symmetry breaking of light quark sea came from the measurements of the Gottfried sum $\left(S_{G}\right)$ in the DIS experiments. The Gottfried sum is defined as

$$
\begin{aligned}
S_{G} & =\int_{0}^{1}\left[F_{2}^{p}(x)-F_{2}^{n}(x)\right] \frac{d x}{x} \\
& =\int_{0}^{1} \sum_{i} e_{i}^{2}\left[q_{i}^{p}(x)+\bar{q}_{i}^{p}(x)-q_{i}^{n}(x)-\bar{q}_{i}^{n}(x)\right] d x \\
& =\frac{1}{3} \int_{0}^{1}\left[u_{v}^{p}(x)-d_{v}^{p}(x)\right] d x+\frac{2}{3} \int_{0}^{1}\left[\bar{u}^{p}(x)-\bar{d}^{p}(x)\right] d x \\
& =\frac{1}{3}+\frac{2}{3} \int_{0}^{1}\left[\bar{u}^{p}(x)-\bar{d}^{p}(x)\right] d x
\end{aligned}
$$

where $x$ is the Bjorken variable, $F_{2}^{p}$ and $F_{2}^{n}$ are the proton and neutron structure functions. Eq. (27) is derived assuming charge symmetry at the partonic level, namely, $u^{p}(x)=d^{n}(x), d^{p}(x)=u^{n}(x), \bar{u}^{p}(x)=$ $\bar{d}^{n}(x)$, and $\bar{d}^{p}(x)=\bar{u}^{n}(x)$. If the nucleon sea is $\bar{u}, \bar{d}$ flavor symmetric, the Gottfried Sum Rule (GSR), $S_{G}=1 / 3$, is obtained.

The New Muon Collaboration (NMC) [19, 20] determined the Gottfried sum to be $0.235 \pm 0.026$ at $Q^{2}=4 \mathrm{GeV}^{2}$, which is significantly below $1 / 3$. This cast doubt on the validity of assuming an symmetric $\bar{u}, \bar{d}$ sea of the proton. Even though the violation of the GSR could also be caused by unusual behavior of the parton distributions at unmeasured small- $x$ region, as well as by the violation of the charge symmetry at the partonic level, this surprising result is usually interpreted as an evidence of flavor asymmetry of the nucleon light sea quarks [21].

\begin{tabular}{lllll}
\hline & CT10 & MSTW2008 & NNPDF2.3 & Experiment \\
\hline \hline$S_{G}\left(4 \mathrm{GeV}^{2}\right)$ & $0.2443(7)$ & $0.2807(4)$ & $0.2394(24)$ & $0.235(26)($ NMC [20]) \\
\hline \hline
\end{tabular}

Table 2: Values of the Gottfried sum, $S_{G}$, for CT10 [16], MSTW2008 [17] and NNPDF2.3 [18] PDFs at $Q^{2}=4 \mathrm{GeV}^{2}$ and from the NMC experiment.

In Table 2 we evaluate the Gottfried sum, $S_{G}$, estimated by the parton distribution functions (PDFs) from some recent global analysis, CT10 [16], MSTW2008 [17] and NNPDF2.3 [18], at $Q^{2}=4 \mathrm{GeV}^{2}$. There are visible differences in $S_{G}$ among them which could be understood from their $x$ distributions of $x(u+\bar{u}-d-\bar{d}) / 3$ and $x(\bar{d}-\bar{u})$ of these PDFs, shown in Figs. 6(a) and 6(b), respectively. Clearly the discrepancy mostly originates from the considerable uncertainties of sea quark densities in both smalland large- $x$ regime, and this calls for the need of exploring the structure of sea quarks by the on-going Fermilab E906/Seaquest Drell-Yan experiment and the proposed electron ion colliders. 


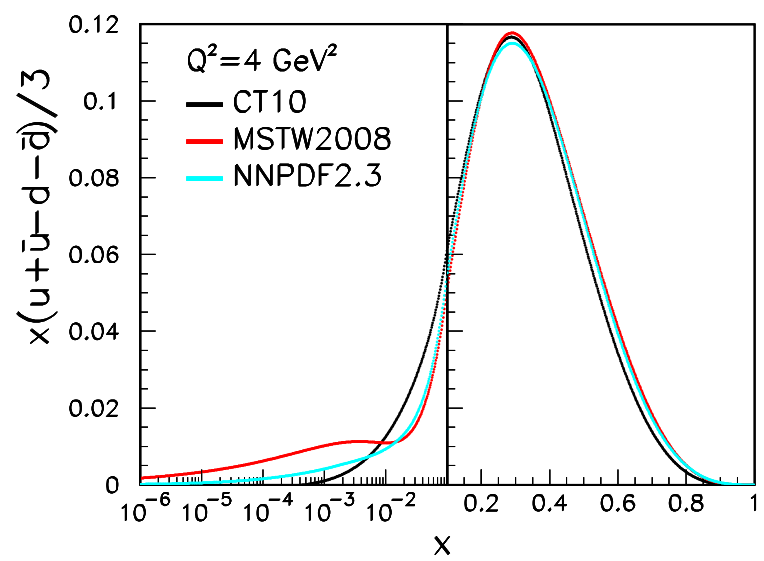

(a)

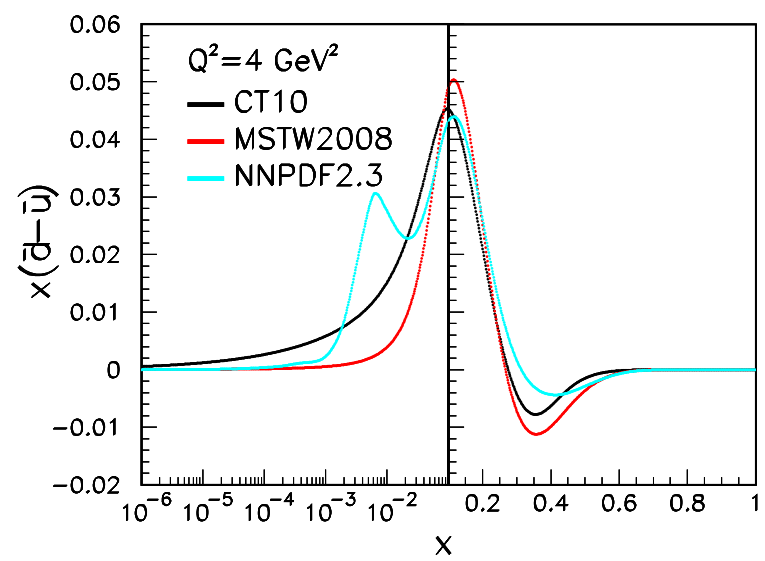

(b)

Figure 6: The $x$ distributions of (a) $x(u+\bar{u}-d-\bar{d}) / 3$, and (b) $x(\bar{d}-\bar{u})$ from CT10 [16], MSTW2008 [17] and NNPDF2.3 [18] PDFs at $Q^{2}=4 \mathrm{GeV}^{2}$.

After the DIS result from NMC, an independent and elegant test of the flavor asymmetry of sea quark was done by the proton-induced Drell-Yan (DY) experiments. The DY cross section at forward rapidity measured by fixed-target experiments is dominated by the annihilation of the $u\left(x_{1}\right)$ quark in the proton beam with the $\bar{u}\left(x_{2}\right)$ antiquark in the target nucleon. For $x_{1} \gg x_{2}$ and $d\left(x_{1}\right) \ll 4 u\left(x_{1}\right)$, the ratio of DY cross sections using deuterium and hydrogen target could be simplified as,

$$
\frac{\sigma^{p d}}{2 \sigma^{p p}} \approx \frac{1}{2} \frac{\left[1+\frac{1}{4} \frac{d\left(x_{1}\right)}{u\left(x_{1}\right)}\right]}{\left[1+\frac{1}{4} \frac{d\left(x_{1}\right)}{u\left(x_{1}\right)} \frac{\bar{d}\left(x_{2}\right)}{\bar{u}\left(x_{2}\right)}\right]}\left[1+\frac{\bar{d}\left(x_{2}\right)}{\bar{u}\left(x_{2}\right)}\right] \approx \frac{1}{2}\left[1+\frac{\bar{d}\left(x_{2}\right)}{\bar{u}\left(x_{2}\right)}\right]
$$

Therefore, the $x$ dependence of $\bar{d} / \bar{u}$ could be determined by the DY process. The NA51 experiment at CERN gave the first result of $\bar{d} / \bar{u}=0.51 \pm 0.04 \pm 0.05$ at $\langle x\rangle=0.18$ and $\left\langle M_{\mu \mu}\right\rangle=5.22 \mathrm{GeV}$ using 450 $\mathrm{GeV}$ proton beam [22]. The Fermilab E866/NuSea experiment later performed the measurement over a broad range of $x$ using $800 \mathrm{GeV}$ proton beam [23]. The extracted $\bar{d} / \bar{u}(x)$ ratios are shown in Fig. 7(a) and they increase linearly from 1 at $x=0$ up to $x \sim 0.15$, reaching a maximum of 1.75 and then drops off at higher $x$. The $\bar{d} / \bar{u}$ ratio falls below unity at the largest $x$. Figure 7 compares $\bar{d}(x) / \bar{u}(x)$ and $\bar{d}(x)-\bar{u}(x)$ with the results of recent PDFs: CT10 [16], MSTW2008 [17] and NNPDF2.3 [18].

The other results on the $\bar{d} / \bar{u}$ flavor asymmetry came from HERMES collaboration [24] where charged pions produced from SIDIS process off hydrogen and deuterium targets in the kinematic regions of $0.02<x<0.3$ and $1<Q^{2}<10 \mathrm{GeV}^{2} / \mathrm{c}^{2}$ were measured. The value of $\bar{d}(x)-\bar{u}(x)$ was deduced from the measured yields as follows:

$$
\frac{\bar{d}(x)-\bar{u}(x)}{u(x)-d(x)}=\frac{J(z)[1-r(x, z)]-[1+r(x, z)]}{J(z)[1-r(x, z)]+[1+r(x, z)]}
$$

where

$$
r(x, z)=\frac{d \sigma_{p}^{\pi^{-}} / d z-d \sigma_{n}^{\pi^{-}} / d z}{d \sigma_{p}^{\pi^{+}} / d z-d \sigma_{n}^{\pi^{+}} / d z}, \text { and } J(z)=\frac{3}{5}\left(\frac{1+D_{u}^{\pi^{-}}(z) / D_{u}^{\pi^{+}}(z)}{1-D_{u}^{\pi^{-}}(z) / D_{u}^{\pi^{+}}(z)}\right)
$$

and $D_{u}^{\pi^{ \pm}}(z)$ are the corresponding favored and disfavored pion fragmentation functions. The ratio $r(x, z)$ was determined from the hydrogen and deuterium data. HERMES's results of $\bar{d}(x)-\bar{u}(x)$ are consistent with E866's ones at larger $Q^{2}$ as shown in Fig. 7(b). 


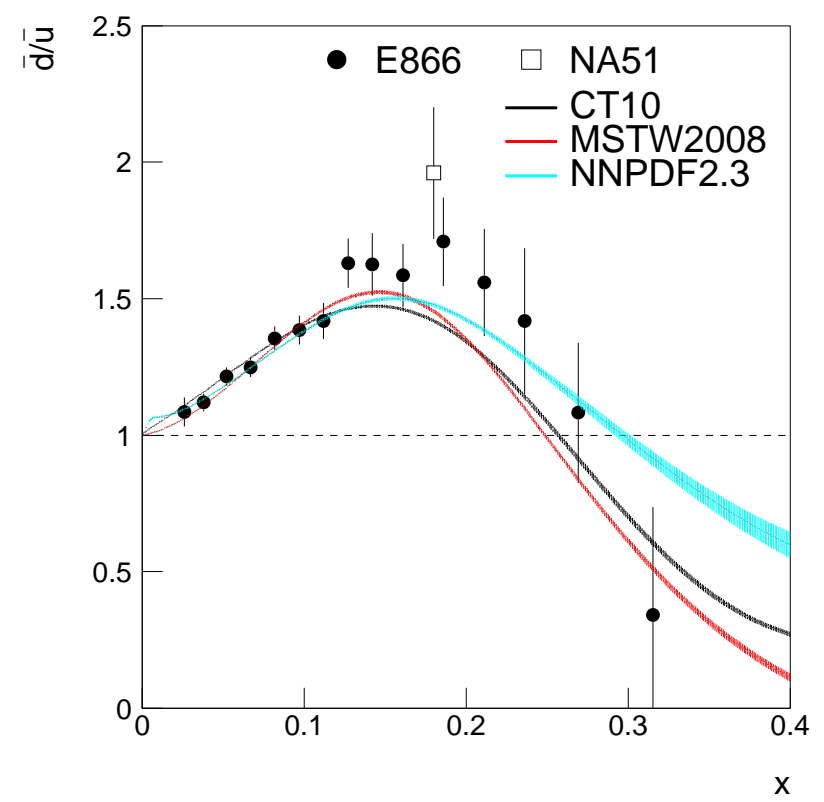

(a)

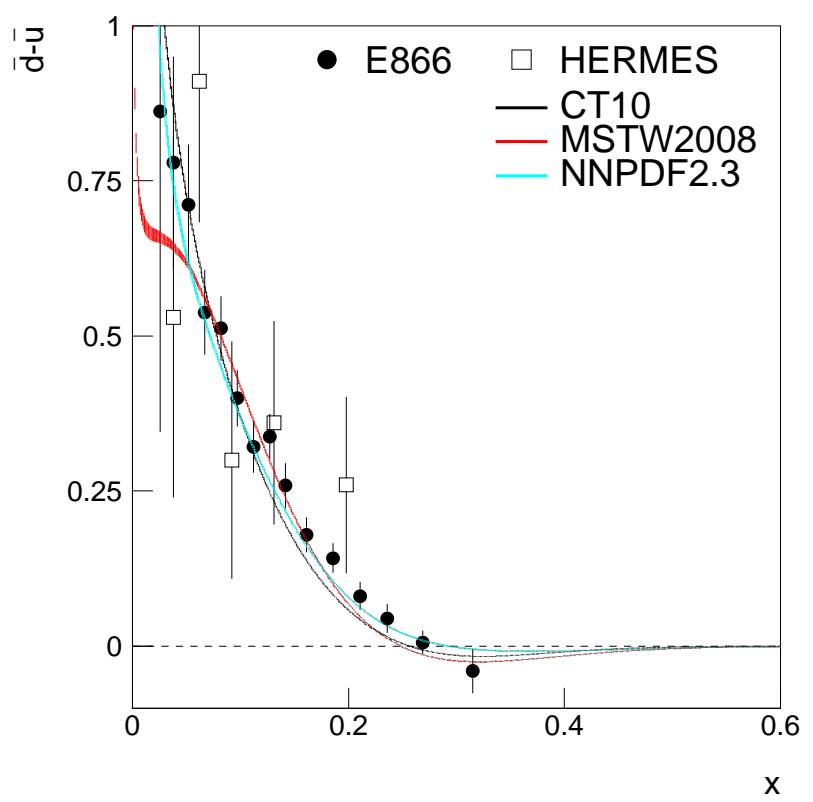

(b)

Figure 7: Data of $(\mathrm{a}) \bar{d}(x) / \bar{u}(x)$ and $(\mathrm{b}) \bar{d}(x)-\bar{u}(x)$ from NA51 [22], E866/NuSea [23] and HERMES [24], compared to the results of CT10 [16], MSTW2008 [17] and NNPDF2.3 [18] PDFs at $Q^{2}=54 \mathrm{GeV}^{2}$.

Table 3 summarizes three experimental determinations of the integral $\int_{0}^{1}(\bar{d}(x)-\bar{u}(x)) d x$ at different energy scales and the corresponding evaluation using various PDFs. This integral calculated with three different PDFs shows very small $Q^{2}$ dependence. The fact that the MSTW2008 integral is much smaller than those of the other two PDFs could be understood from the structure of $\bar{d}(x)-\bar{u}(x)$ displayed in Fig. 6(b), The E866 integral is smaller than those from NMC and HERMES, but consistent with them within the quoted errors.

\begin{tabular}{lllll}
\hline$\left\langle Q^{2}\right\rangle$ & CT10 & MSTW2008 & NNPDF2.3 & Experiment \\
\hline \hline $2.5 \mathrm{GeV}^{2}$ & $0.129(1)$ & $0.079(1)$ & $0.137(3)$ & $0.16(3)($ HERMES [24]) \\
$4 \mathrm{GeV}^{2}$ & $0.129(1)$ & $0.079(1)$ & $0.138(3)$ & $0.148(39)($ NMC [20]) \\
$54 \mathrm{GeV}^{2}$ & $0.130(1)$ & $0.080(1)$ & $0.139(3)$ & $0.118(12)(\mathrm{E} 866[23])$ \\
\hline \hline
\end{tabular}

Table 3: Energy scale $\left(\left\langle Q^{2}\right\rangle\right)$ and the corresponding estimation of $\int_{0}^{1}(\bar{d}(x)-\bar{u}(x)) d x$ using CT10 [16], MSTW2008 [17] and NNPDF2.3 [18] PDFs together with the results determined by three experiments.

Many theoretical attempts have been made to understand the origin of the nucleon sea and the flavor asymmetry. In 1977 Field and Feynman 25] pointed out that the $\bar{d}=\bar{u}$ would not strictly hold in the perturbative QCD because the additional valence $u$ quark in proton could lead to a larger suppression of $g \rightarrow u \bar{u}$ via Pauli blocking. This provided a simple and qualitative interpretation of the observed flavor asymmetry of $\bar{d}$ and $\bar{u}$ in the nucleons. However later NLO perturbative QCD calculations confirmed [26] that the Pauli blocking effect in the gluon splitting is too small to account for the sizable violation of GSR. Hence it is generally believed that the observed large difference between $\bar{d}(x)$ and $\bar{u}(x)$ suggests a non-perturbative origin.

The observed $\bar{d} / \bar{u}$ asymmetry could be reasonably explained by meson-cloud model, chiral quark model, chiral quark soliton model, and instanton model. Details of these models have been reviewed 
in several articles [27, 28, 29, 30]. For example, the meson-cloud model [31, 32] treats the proton as a linear combination of a bare proton plus pion-nucleon and pion-delta states:

$$
\begin{aligned}
|p\rangle & =\sqrt{Z}\left|p_{0}\right\rangle+a_{N \pi / p}\left[-\sqrt{\frac{1}{3}}\left|p_{0} \pi^{0}\right\rangle+\sqrt{\frac{2}{3}}\left|n_{0} \pi^{+}\right\rangle\right] \\
& +a_{\Delta \pi / p}\left[\sqrt{\frac{1}{2}}\left|\Delta_{0}^{++} \pi^{-}\right\rangle-\sqrt{\frac{1}{3}}\left|\Delta_{0}^{+} \pi^{0}\right\rangle+\sqrt{\frac{1}{6}}\left|\Delta_{0}^{0} \pi^{+}\right\rangle\right] \\
& +a_{\Lambda K / p}\left|\Lambda_{0} K^{+}\right\rangle+a_{\Sigma K / p}\left[-\sqrt{\frac{1}{2}}\left|\Sigma_{0}^{+} K^{0}\right\rangle+\sqrt{\frac{1}{2}}\left|\Sigma_{0}^{0} K^{+}\right\rangle\right]+\ldots
\end{aligned}
$$

where the subscript zeros denote bare baryons with flavor symmetric seas and $Z$ is the normalization constant and the parameter $a_{B M / p}$ reflects the relative strength of proton splitting into the virtual baryon-meson (BM) state. The $x$ distributions of sea quarks $\bar{u}, \bar{d}, s$ and $\bar{s}$ could be obtained by convoluting their distributions in either meson or baryon with the splitting functions. The $\bar{u}$ and $\bar{d}$ seas receive contributions from the valence antiquarks of the pion cloud. The excess of $\bar{d}$ over $\bar{u}$ arises because of the dominance of the $n_{0} \pi^{+}$configuration over the less probable $\Delta_{0}^{++} \pi^{-}$configuration. This leads to an overall excess of $\bar{d}$ over $\bar{u}$. Similarly the different distributions of $\bar{s}(x)$ in the fluctuating kaon, and $s(x)$ in the $\Lambda$ and $\Sigma$ hyperons could introduce possible $s(x) / \bar{s}(x)$ asymmetries in the proton.

In the chiral quark model [33] the relevant degrees of freedom are constituent quarks and Goldstone bosons $(\pi, K, \eta)$. The Goldstone bosons directly couple to the constituent quarks of the proton as a consequence of the spontaneously broken chiral symmetry at low energies. The Fock decomposition of the constituent quarks $|U\rangle$ and $|D\rangle$ in the proton could be represented as follows:

$$
\begin{aligned}
& |U\rangle=\sqrt{Z}|u\rangle+\sqrt{\frac{1}{3}} a_{\pi / U}\left|u \pi^{0}\right\rangle+\sqrt{\frac{2}{3}} a_{\pi / U}\left|d \pi^{+}\right\rangle+a_{K / U}\left|s K^{+}\right\rangle+\ldots \\
& |D\rangle=\sqrt{Z}|d\rangle+\sqrt{\frac{1}{3}} a_{\pi / D}\left|d \pi^{0}\right\rangle+\sqrt{\frac{2}{3}} a_{\pi / D}\left|u \pi^{-}\right\rangle+a_{K / D}\left|s K^{0}\right\rangle+\ldots
\end{aligned}
$$

where small letters denote bare constituent quarks. The $Z$ and $a_{M / q}$ are the normalization constant and parameter of Goldstone bosons $(M)$ coupling with the bare constituent quark states. The descriptions of the asymmetries of $\bar{d}(x) / \bar{u}(x)$ and $s(x) / \bar{s}(x)$ could be derived in similar manners as what is done in the meson-cloud model.

In the past decade, there are updated results from meson-cloud model [34], chiral quark model [35, 36, 37], and chiral quark soliton model [38, 39, 40]. In Ref. [34], Gaussian forms for the momentum distributions and hadronic fluctuations of baryons into baryon-meson pairs were used in the framework of meson-cloud model and proper QCD evolution is taken into account. The asymmetry of $\bar{u}(x)$ and $\bar{d}(x)$ found in the Drell-Yan ratio of $p p$ and $p d$ scattering can be properly described. The chiral soliton model has been generalized into the flavor SU(3) version [38] and the SU(3) breaking effect due to the mass difference $\delta m_{s}$ between the $s$ quark and the $u, d$ quarks is estimated using first order perturbation theory in the parameter $\delta m_{s}$. The magnitude of the asymmetry $\bar{d}(x)-\bar{u}(x)$ remains the same after this $\mathrm{SU}(3)$ generalization.

There are two new attempts to understand the sea flavor asymmetry putting emphasis on the statistical properties of partons bound inside the nucleon: statistical model and balance model. Bourrely et al. [41, 42, 43, 44, 45] developed a new form of statistical parametrization, allowing an $x$-dependent chemical potential. By incorporating QCD evolution they can describe a variety of data. Zhang et al. [46, 47, 48, 49, 50] constructed a model using the principle of detailed balance without any free parameter, and they obtained Gottfried sum in surprisingly good agreement with the experimental values. 
Figure 8 shows various theoretical attempts in describing the data of $\bar{d}(x) / \bar{u}(x)$ and $\bar{d}(x)-\bar{u}(x)$. In common, all the models could describe the general feature of $\bar{d}>\bar{u}$ reasonably well but none of them could accommodate the tentative behavior of $\bar{d}(x) / \bar{u}(x)<1$ at large $x$.

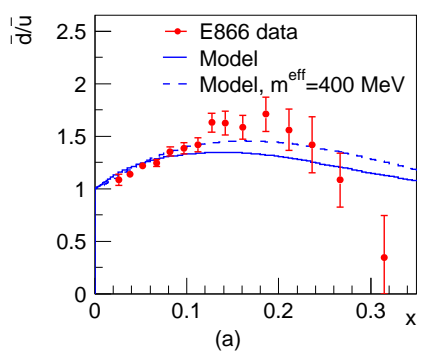

(a) Meson cloud model. Figure from 34
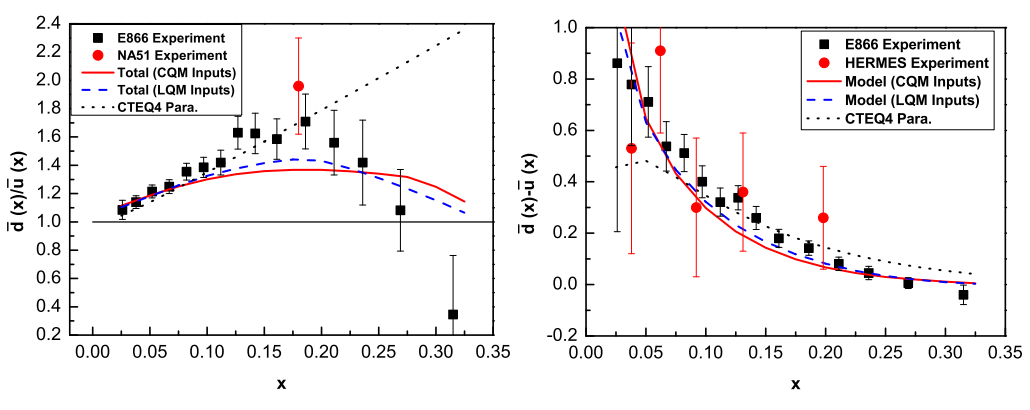

(b) Chiral quark model. Figure from 37
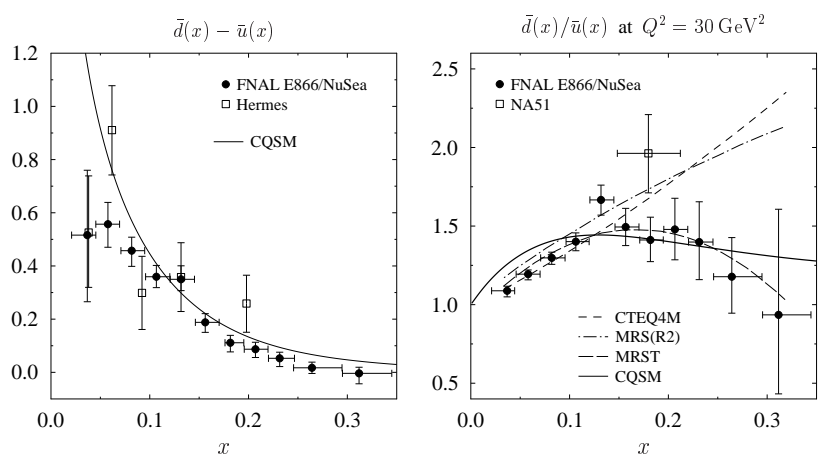

(c) Chiral quark soliton model. Figure from 40]

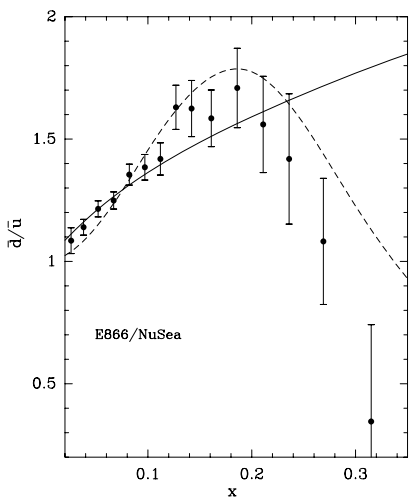

(d) Statistic model.

Figure from 41 .

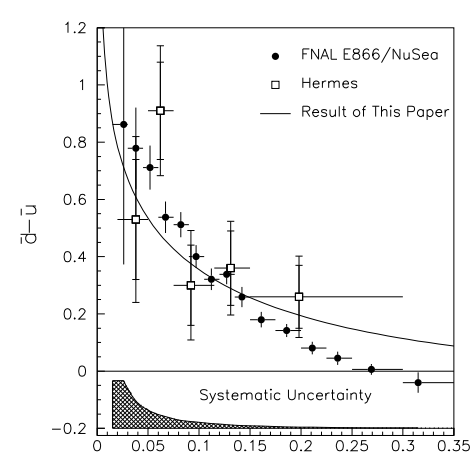

(e) Balance model.

Figure from [47]

Figure 8: Comparison of the data of $\bar{d}(x) / \bar{u}(x)$ and $\bar{d}(x)-\bar{u}(x)$ from NA51 [22], E866/NuSea [23] and HERMES [24] with the predictions of theoretical models.

Thomas et al. [51] have derived a leading non-analytic chiral behavior of $\bar{d}-\bar{u}$ which is a unique characteristics of Goldstone boson loops in chiral theories. It provided a possible theoretical connection between the flavor asymmetry in the nucleon sea and the fundamental chiral symmetry breaking in QCD. There exists difficulty in exploring the sea structure from lattice QCD: only lower moments of the parton distributions could be computed and the separation of quark and antiquark distributions is not available. Very recently, the qualitative feature of the nucleon sea flavor structure of $\bar{d}(x)>\bar{u}(x)$ (and also $\Delta \bar{d}(x)<\Delta \bar{u}(x)$ ) was demonstrated directly from QCD by the lattice approach with a large pion mass of $310 \mathrm{MeV}$ [52], as shown in Fig. 9. By doing calculations with a large-momentum nucleon, the light-cone quantities are connected to lattice-QCD non-local time-independent matrix elements [53]. These results are encouraging and promising for a realistic comparison with the measured $x$ dependence of sea quark structure in the future. 


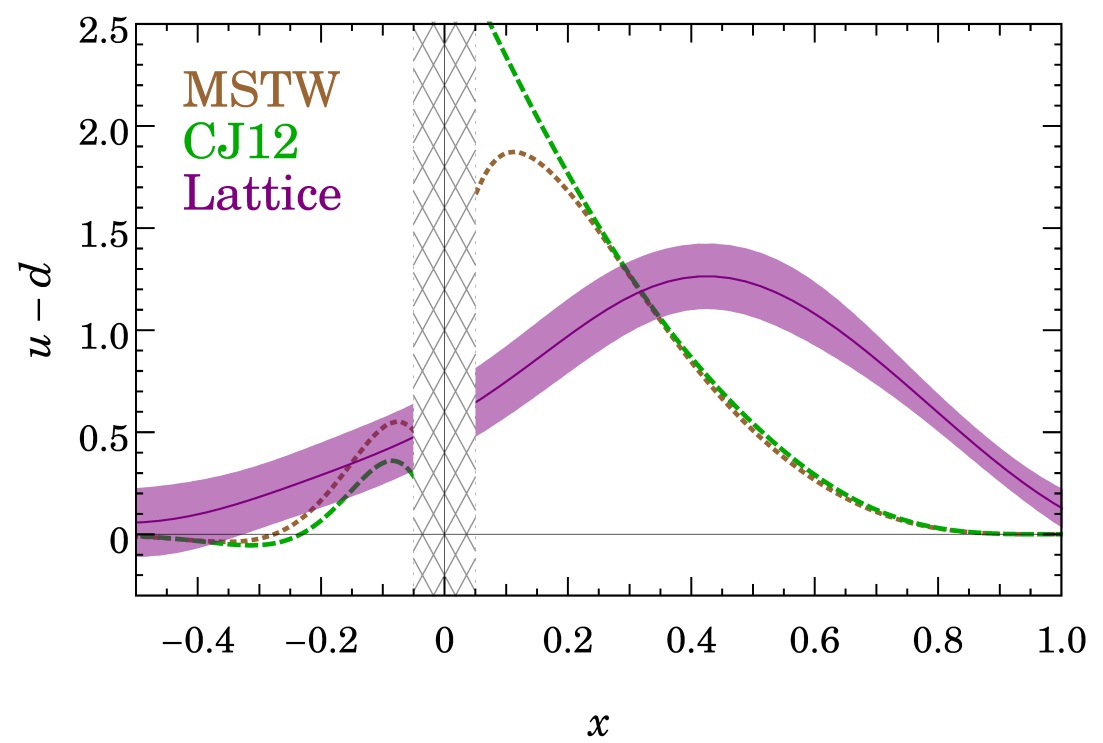

Figure 9: Unpolarized isovector quark distributions computed by lattice QCD [52](purple band), compared with the global analysis of MSTW2008 [17](brown dotted line) and CJ12 [54](green dashed line) PDFs. The sea quark distribution is represented in the negative $x$ region as $\bar{q}(x)=-q(-x)$. Figure from [52].

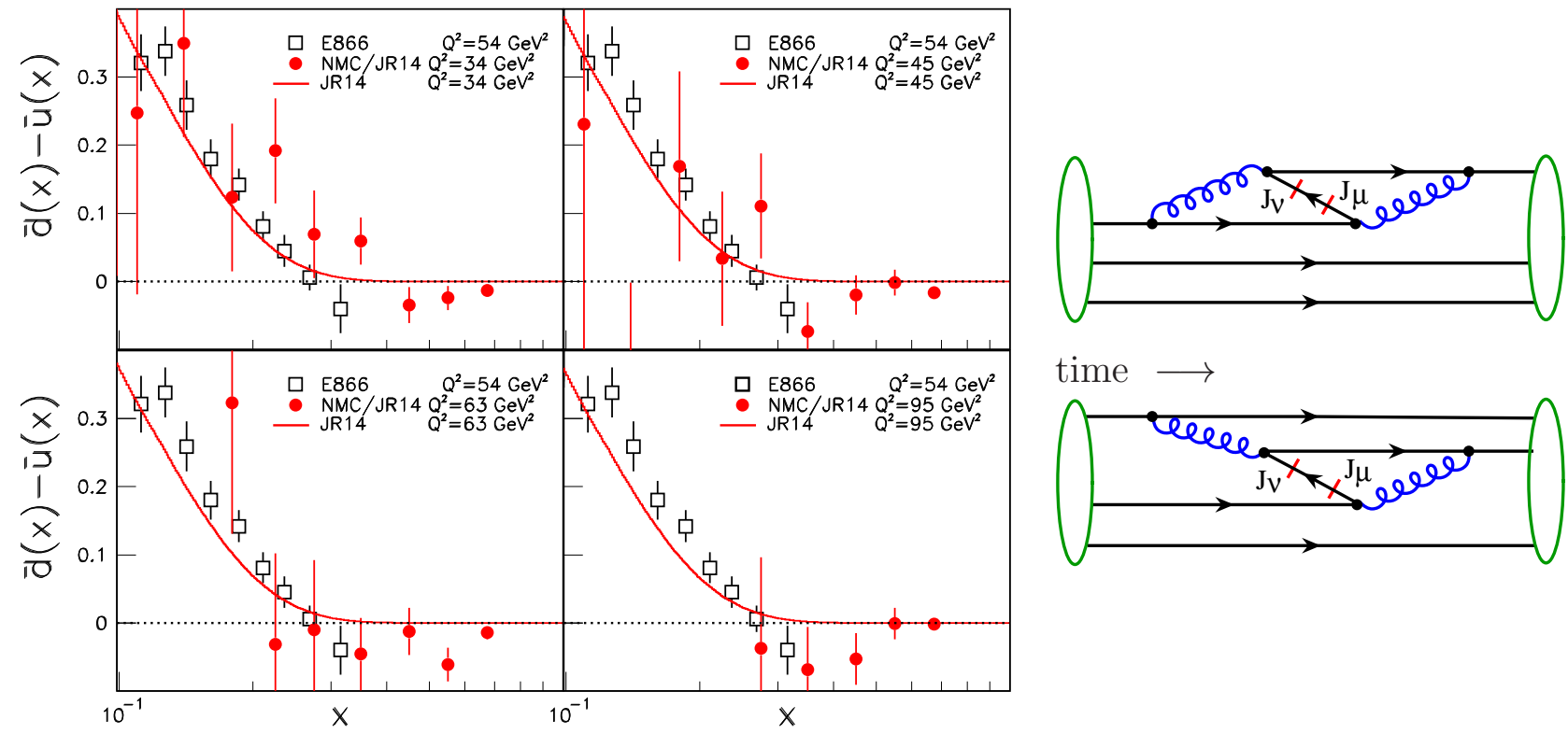

Figure 10: Left (a): Values of $\bar{d}(x)-\bar{u}(x)$ evaluated using Eq. (33) and the NMC data [57, 58] of $F_{2}^{p}(x)-F_{2}^{n}(x)$ at $Q^{2}=34,45,63$, and $95 \mathrm{GeV}^{2}$. The JR14 parametrization for $u_{v}(x)-d_{v}(x)$ is used. The values of $\bar{d}(x)-\bar{u}(x)$ from E866 measurement at $Q^{2}=54 \mathrm{GeV}^{2}$ are also shown. The solid curves are $\bar{d}(x)-\bar{u}(x)$ from JR14. Right (b): QCD quantum fluctuation capable of generating connected $\bar{u}(x)$ or $\bar{d}(x)$, involving one (top) or two (bottom) valence quarks, which could lead to more $\bar{u}(x)$ than $\bar{d}(x)$. Figure from [55].

Recently an independent evidence was reported for the $\bar{d}(x)-\bar{u}(x)$ sign-change at $x \sim 0.3$ based on an analysis of the NMC DIS data [55]. At the leading order in $\alpha_{s}$ the $\bar{d}(x)-\bar{u}(x)$ can be extracted 
from the NMC measurement of $F_{2}^{p}(x)-F_{2}^{n}(x)$ and the parametrization of $u_{v}(x)-d_{v}(x)$ from the PDF as follows:

$$
\bar{d}(x)-\bar{u}(x)=\frac{1}{2}\left[u_{v}(x)-d_{v}(x)\right]-\frac{3}{2 x}\left[F_{2}^{p}(x)-F_{2}^{n}(x)\right] .
$$

Figure 10(a) shows $\bar{d}(x)-\bar{u}(x)$ at four values of $Q^{2}$ using Eq. 33] with the NNLO JR14 [56] parametrization of the valence quark distributions and the NMC data [57, 58] for $F_{2}^{p}(x)-F_{2}^{n}(x)$. The JR14 is a recent PDF where the nuclear corrections from the CJ group [59] is implemented and $\bar{d}(x)-\bar{u}(x)>0$ is assumed at all $x$ in the global analysis. Very similar results are obtained if more recent PDFs are used for the valence quark distributions. Figure 10(a) shows that both the NMC and the E866 data show evidence that $\bar{d}(x)-\bar{u}(x)$ changes sign at $x \sim 0.3$.

Specific examples of diagrams possibly responsible for generating more $\bar{u}$ than $\bar{d}$ at high $x$ involving one or two valence quarks are shown in Fig.10(b). The antiquark mode of the QCD quantum fluctuation of a quark is probed by the currents $J_{\mu}$ and $J_{\nu}$. In time sequence the valence quark first radiates a highly virtual gluon which splits into a quark-antiquark pair. It is followed by the annihilation or recombination of the valence quark and the newly produced antiquark into a highly virtual gluon, which is finally absorbed by the quark. These diagrams could generate roughly a factor of 2 more $\bar{u}(x)$ than $\bar{d}(x)$ due to $u_{v} / d_{v}$ the 2 -to- 1 ratio of valence quarks in the proton.

The significance of the sign-change of $\bar{d}(x)-\bar{u}(x)$ for $x>0.3$, if confirmed by future experiments, e.g. E906/SeaQuest Drell-Yan experiment, is that it would severely challenge existing theoretical models which can successfully explain $\bar{d}(x)-\bar{u}(x)$ at $x<0.25$, but predict no sign-change at higher $x$.

\subsection{Strange Quark Sea}

\subsubsection{Breaking of SU(3) flavor symmetry of quark sea}

Most of the information about the strange sea is obtained from the neutrino deep inelastic scattering [60, 61] via the process of $W$ boson exchange as introduced in Sec. 2.2. The measurements of charm production with dimuon events in the final state have been performed by several experiments: CDHS [62, CCFR [63, 64, CHARMII [65], NOMAD [66, 67], NuTeV [68, 69, 70, 71] and CHORUS [72, 73]. The integrated strange over non-strange nucleon sea $\kappa_{s}$, defined as

$$
\kappa_{s}=\frac{\int_{0}^{1} x\left[s\left(x, \mu^{2}\right)+\bar{s}\left(x, \mu^{2}\right)\right] d x}{\int_{0}^{1} x\left[\bar{u}\left(x, \mu^{2}\right)+\bar{d}\left(x, \mu^{2}\right)\right] d x},
$$

and the strange sea over non-strange quark, $\eta_{s}$, defined as

$$
\eta_{s}=\frac{\int_{0}^{1} x\left[s\left(x, \mu^{2}\right)+\bar{s}\left(x, \mu^{2}\right)\right] d x}{\int_{0}^{1} x\left[u\left(x, \mu^{2}\right)+d\left(x, \mu^{2}\right)\right] d x},
$$

are determined by these experiments at the energy scale $\mu^{2}$ and the results are summarized in Table 4 . It is noted that the extracted values of $\kappa_{s}$ and $\eta_{s}$ depend on the order of perturbative QCD correction for charm quark production and the charm fragmentation function. Since the determination was done with several parameters simultaneously, $\kappa_{s}$ or $\eta_{s}$ also correlates with the other parameters e.g. the mass of charm $m_{c}$ and the branching ratio $B_{c}$.

The most recent result of $\kappa_{s}$ is $0.591 \pm 0.019$ at $\mu^{2}=20 \mathrm{GeV}^{2}$ determined at NNLO by NOMAD collaboration [67]. Overall the results of $\kappa_{s}$ indicate that the momentum fraction carried by strange sea is about $30-50 \%$ of that carried by non-strange sea quarks and the $\mathrm{SU}(3)$ flavor symmetry is broken.

The momentum fraction ratio of the strange quark to the total non-strange quark content, $\eta_{s}$, is about $5-10 \%$. 


\begin{tabular}{|c|c|c|c|c|c|}
\hline Experiment (year) & QCD order & $\kappa_{s}$ & $\eta_{s}$ & $\mu^{2}\left(\mathrm{GeV}^{2}\right)$ & Ref. \\
\hline CDHS (1982) & LO & $0.52 \pm 0.09$ & $0.052 \pm 0.004$ & 20 & 62 \\
\hline CCFR (1993) & LO & $0.373_{-0.041}^{+0.048} \pm 0.018$ & $0.064_{-0.007}^{+0.008} \pm 0.002$ & 22.2 & 63 \\
\hline CCFR (1995) & NLO & $0.477_{-0.050+0.036}^{+0.051-0.017}$ & $0.099 \pm 0.008 \pm 0.004$ & 22.2 & 64] \\
\hline CHARMII (1999) & LO & $0.388_{-0.0161}^{+0.074} \pm 0.067$ & $0.068 \pm 0.014$ & 20 & 65 \\
\hline NOMAD (2000) & $\mathrm{LO}$ & $0.48_{-0.07-012}^{+0.09+17}$ & $0.071_{-0.009-0.015}^{+0.011+0.020}$ & & 66 \\
\hline $\mathrm{NuTeV}(2001)$ & $\mathrm{LO}$ & $0.38 \pm 0.08 \pm 0.043$ & & & 68 \\
\hline NuTeV (2007) & NLO & & $0.061 \pm 0.001 \pm 0.006$ & & 71 \\
\hline CHORUS (2008) & NLO & $0.33 \pm 0.05 \pm 0.05$ & & 20 & 72 \\
\hline NOMAD (2013) & NNLO & $0.591 \pm 0.019$ & & 20 & 67 \\
\hline
\end{tabular}

Table 4: List of neutrino-induced dimuon experiments, the order of $\mathrm{QCD}$ analysis for charm quark production, the strange quark content parameters $\kappa_{s}$ and $\eta_{s}$ and the energy scale $\left(\mu^{2}\right)$, in the order of the year of published results. Errors are statistical and systematic.

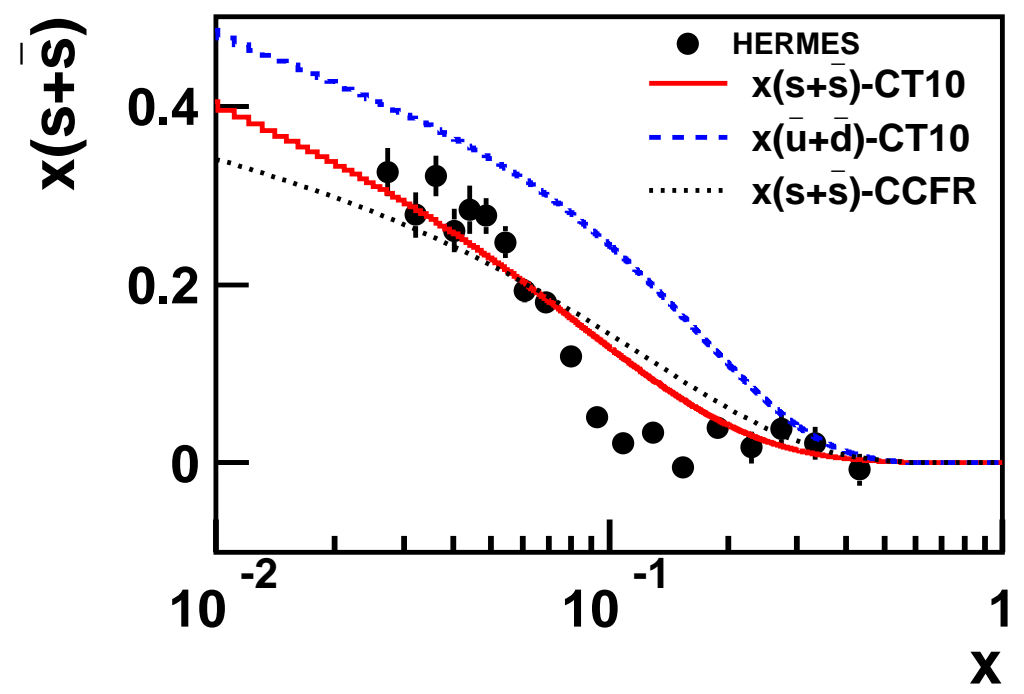

Figure 11: Data of $x(s(x)+\bar{s}(x))$ from HERMES [74] at $Q^{2}=2.5 \mathrm{GeV}^{2}$ in comparison with the predictions of $x(s(x)+\bar{s}(x))$ and $x(\bar{u}(x)+\bar{d}(x))$ from CT10 [16] at $Q^{2}=2.5 \mathrm{GeV}^{2}$ and $x(s(x)+\bar{s}(x))$ from CCFR [64] at $Q^{2}=1 \mathrm{GeV}^{2}$.

In SIDIS reaction, the HERMES collaboration reported the determination of $x(s(x)+\bar{s}(x))$ over the range of $0.02<x<0.5$ at $Q^{2}=2.5 \mathrm{GeV}^{2}$ from their measurement of charged kaon production on a deuteron target [74]. The extraction of strange quark densities is done with leading-order expression. The HERMES data, shown in Fig. 11, exhibits an intriguing feature that $x(s(x)+\bar{s}(x))$ for $x<0.1$ shows a strong rise towards small $x$ and gradually deviates from the parametrization of CCFR at $Q^{2}=1 \mathrm{GeV}^{2}$ [64] but agrees with the prediction of CT10 [16], which does not require $(s(x)+\bar{s}(x))=$ $(\bar{u}(x)+\bar{d}(x)) / 2$ for the parton densities at the initial scale. The $x(s(x)+\bar{s}(x))$ for $x<0.05$ actually become close to $x(\bar{u}(x)+\bar{d}(x))$ of CT10 PDF. This reflects a restoration of SU(3) flavor symmetry at small $x$ even at low $Q^{2}$. Another intriguing feature is that beyond $x \sim 0.1$ the data become relatively independent of $x$ and HERMES results suggest the presence of two different components of the strange sea, one of which dominates at small $x(x<0.1)$ and the other at larger $x(x>0.1)$. Recently HERMES results have been re-evaluated [75], however the results strongly depend on the kaon fragmentation 
function in the extraction process. The strange quark sea has been interpreted as a mixture of intrinsic and extrinsic sea components, to be discussed in Sec 3.3.1. The role of the strange sea in separating the light-quark connected sea from disconnected sea is discussed in Sec 3.3 .2

At LHC, the measurement of $W^{ \pm}$boson production in proton-proton collisions, either inclusive [76] or associated with a single charm quark [77, 78] allows the determination of the strange parton distributions in conjunction with DIS data. First the ATLAS experiment determined the strange-to-down antiquark ratio $r_{s}(=(s+\bar{s}) / 2 \bar{d})$ to be $1.00_{-0.28}^{+0.25}$ at $x=0.023$ and $Q^{2}=1.9 \mathrm{GeV}^{2}$ from the global analysis of inclusive $W$ and $Z$ production in $p p$ collisions at $7 \mathrm{TeV}$ and DIS inclusive data from HERA (ATLAS-epWZ12) [79. The result supports the hypothesis of an SU(3)-symmetric light-quark sea, i.e $\bar{s}=\bar{d}$ in the small $x$ region at small $Q^{2}$, consistent with what was observed by HERMES in SIDIS reaction.

Recently ATLAS determined the same ratio $r_{s}$ to be $0.96_{-0.30}^{+0.26}$ at $Q^{2}=1.9 \mathrm{GeV}^{2}$ using the measurement of cross sections of the associated $W+c$ production at LHC [80] and the DIS data from HERA (represented by HERAPDF1.5 [81]), in good agreement with the values determined in the previous combined analysis of $W, Z$ production. Figure 12 shows the $x$-dependence of $r_{s}$ at $Q^{2}=m_{W}^{2}$, obtained from three global analyses: HERAPDF1.5, ATLAS-epWZ12 and HERAPDF1.5+ATLAS-Wc. At large $Q^{2}=m_{W}^{2}$, the sea quarks are dominantly produced by QCD evolution and $\mathrm{SU}(3)$ symmetry is clearly observed as seen in the $r_{s}(x) \sim 1$ across the whole $x$ region.

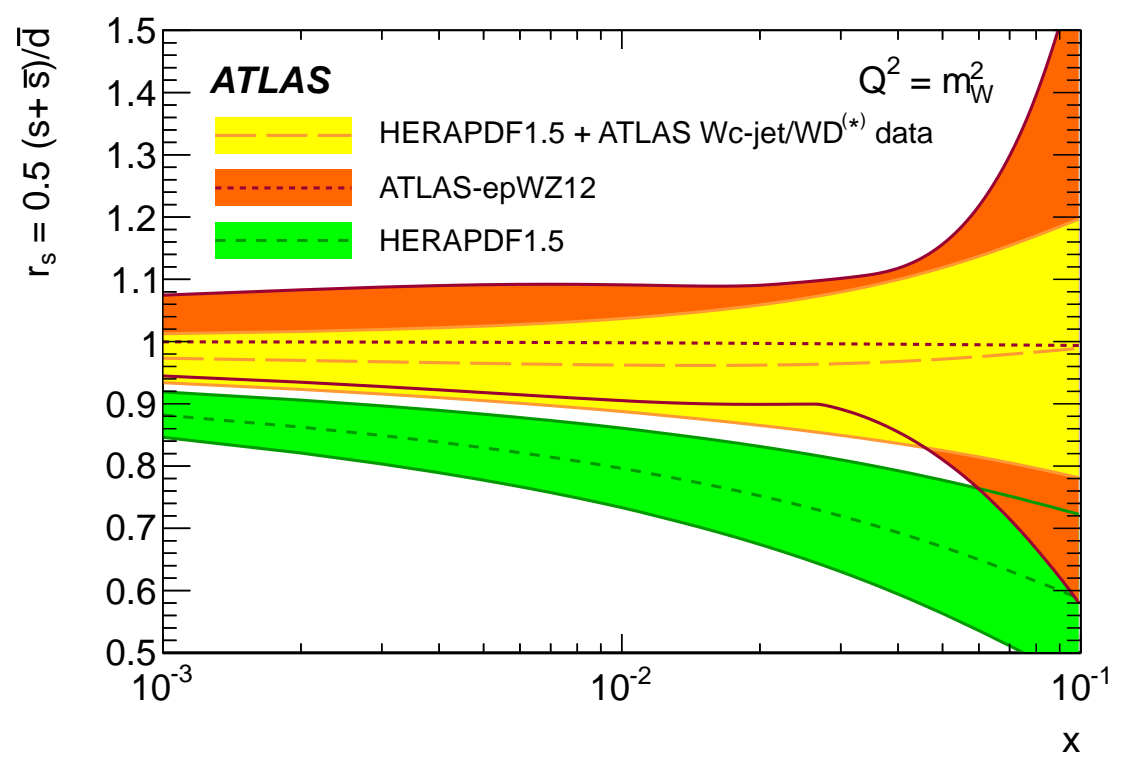

Figure 12: Ratio of strange-to-down sea-quark distributions $r_{s}=0.5(s+\bar{s}) / \bar{d}$ as a function of $x$ at $Q^{2}=M_{W}^{2}$ obtained from HERA data only (HERAPDF1.5), ATLAS $W, Z$ production only (ATLASepWZ12) and HERA plus ATLAS $W+c$ production (HERAPDF1.5+ATLAS-Wc). Figure from [80].

The same measurement of associated $W+c$ production from proton-proton collision at LHC is also done by the CMS collaboration [82. Recently CMS performed a next-to-leading-order global analysis using this result together with those of muon charge asymmetry in the inclusive $W$ production and the inclusive DIS cross sections at HERA for the determination of valence quark and the strange quark distributions [83]. The integrated strange fraction of the nucleon sea $\kappa_{s}$ is determined at $Q^{2}=20 \mathrm{GeV}^{2}$ to be $0.52_{-0.10}^{+0.12}$ (exp. $)_{-0.06}^{+0.05}$ (model $)_{-0.10}^{+0.13}$ (parametrization), in agreement with the value obtained by the neutrino-DIS NOMAD experiment [67].

The strange quark distribution $s\left(x, Q^{2}\right)$ and the strangeness ratio $R_{s}(x, Q)=(s+\bar{s}) /(\bar{u}+\bar{d})\left(x, Q^{2}\right)$ at $Q^{2}=1.9 \mathrm{GeV}^{2}$ and $m_{W}^{2}$ are shown in Fig. 13. The strange quark fraction rises with the energy scale and the $s$ quark becomes comparable to $\bar{u}$ and $\bar{d}$ at the regions of intermediate to low $x$. In the left plot 
of Fig. 13 the $r_{s}$ determined by ATLAS is displayed together with the distributions of $R_{s}(x)$ by CMS at the same scale $Q^{2}=1.9 \mathrm{GeV}^{2}$. Since the difference of $\bar{u}$ and $\bar{d}$ at this $x$ region is expected to be very minor, this comparison shows a $2 \sigma$ difference in the strangeness fraction determined by the LHC experiments. A discussion on the possible cause for this discrepancy is given in Sec. 3.2.3.
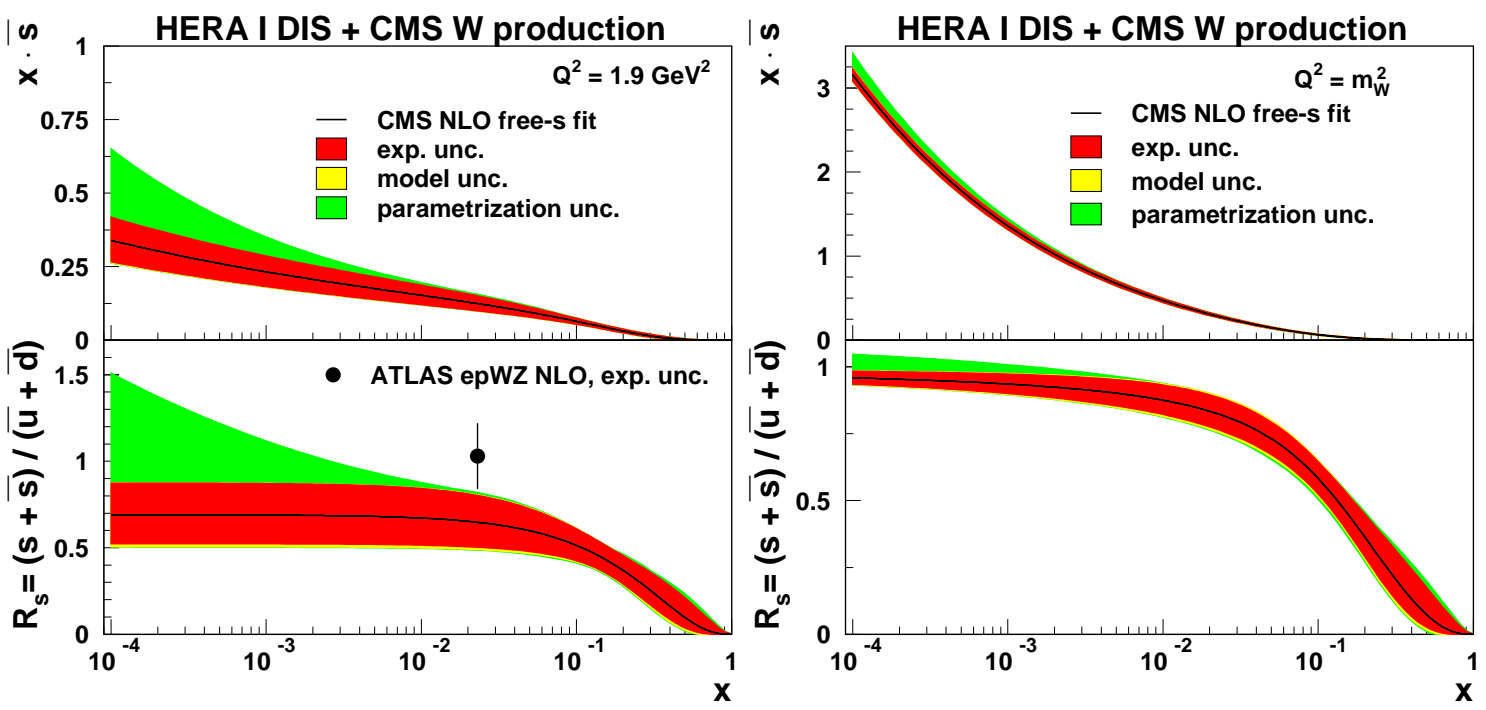

Figure 13: Antistrange-quark distribution $x \bar{s}(x, Q)$ and the strangeness ratio $R_{s}(x, Q)$, obtained in the QCD analysis of HERA and CMS data, shown as functions of $x$ at $Q^{2}=1.9 \mathrm{GeV}^{2}$ (left) and $Q^{2}=M_{W}^{2}$ (right). The NLO ATLAS result of $r_{s}=(s+\bar{s}) / 2 \bar{d}[79]$ is presented by a closed symbol in the left-bottom figure for comparison. Figure from [83].

\subsubsection{Asymmetry between $s(x)$ and $\bar{s}(x)$}

There is no fundamental reason why the $x$ distributions of strange quark and antistrange quark in the nucleons are the same as long as the integral of the asymmetry over all values of $x$ vanishes, i.e. $\langle s-\bar{s}\rangle=\int_{0}^{1}[s(x)-\bar{s}(x)] d x=0$. The strangeness asymmetry could in principle affect the extraction of the Weinberg angle from neutrino-nucleus deep inelastic scattering and thus non-zero asymmetry could partially explain the anomaly seen by the $\mathrm{NuTeV}$ experiment [69] in their measurement of Weinberg angle [34, 35, 39, 84, 85, 86, 87].

The CCFR NLO evaluation of the strange quark distributions did not exclude the possible difference between $s(x)$ and $\bar{s}(x)$ [64]. The NuTeV collaboration determined $\langle x(s-\bar{s})\rangle$ from LO analysis of dimuon events from neutrino (antineutrino) DIS with the nucleon [70 and found a negative value of $-0.0027 \pm 0.0013$. Afterward it performed a complete NLO analysis [71] and found a slightly positive asymmetry of $0.00196 \pm 0.00046 \pm 0.00045$, as shown in Fig. 14. Clearly there is still a large uncertainty in the extraction of $s(x)$ and $\bar{s}(x)$ distributions from the experimental data. In Ref. [88] it was suggested that heavy-quark recombination effect where a heavy $c$ quark combines with a light antiquark forming a $D$ meson should be taken into account in extracting the strange asymmetry from the dimuon events in neutrino (antineutrino) DIS. Their study showed that the value of $\langle x(s-\bar{s})\rangle$ could increase by 0.0023 with the inclusion of this effect. 


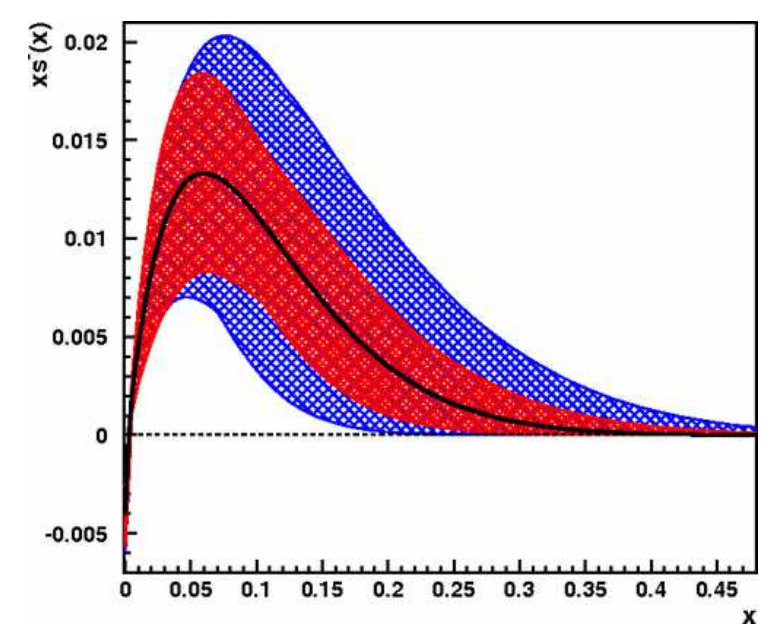

Figure 14: The extracted distribution of $x s^{-}(x)=x(s(x)-\bar{s}(x))$ at $Q^{2}=16 \mathrm{GeV}^{2}$ by NuTeV. Blue error band represents the total uncertainty while the red band is the uncertainty without the error of charm semileptonic branching ratio. Figure from [71].

Using the 3-loop splitting functions [89], a non-zero $s(x)-\bar{s}(x)$ could be generated from NNLO QCD evolution starting with the condition of $s(x)=\bar{s}(x)$ at the initial scale [90]. The reason is that the probability of a $q \rightarrow q^{\prime}$ splitting is different from that of $q \rightarrow \bar{q}^{\prime}$ and the $u, d$ valence densities in the nucleon are different. Figure 15 (a) and (b) shows that the generated asymmetry $s-\bar{s}$ is not negligible which is positive at small $x$ and negative at large $x$. The second moment $\langle x(s-\bar{s})\rangle$ is evaluated to be about $-5 \times 10^{-4}$ at $Q^{2}=20 \mathrm{GeV}^{2}$. The perturbative effect gives a $\langle x(s-\bar{s})\rangle$ with a sign opposite to the latest $\mathrm{NuTeV}$ result.
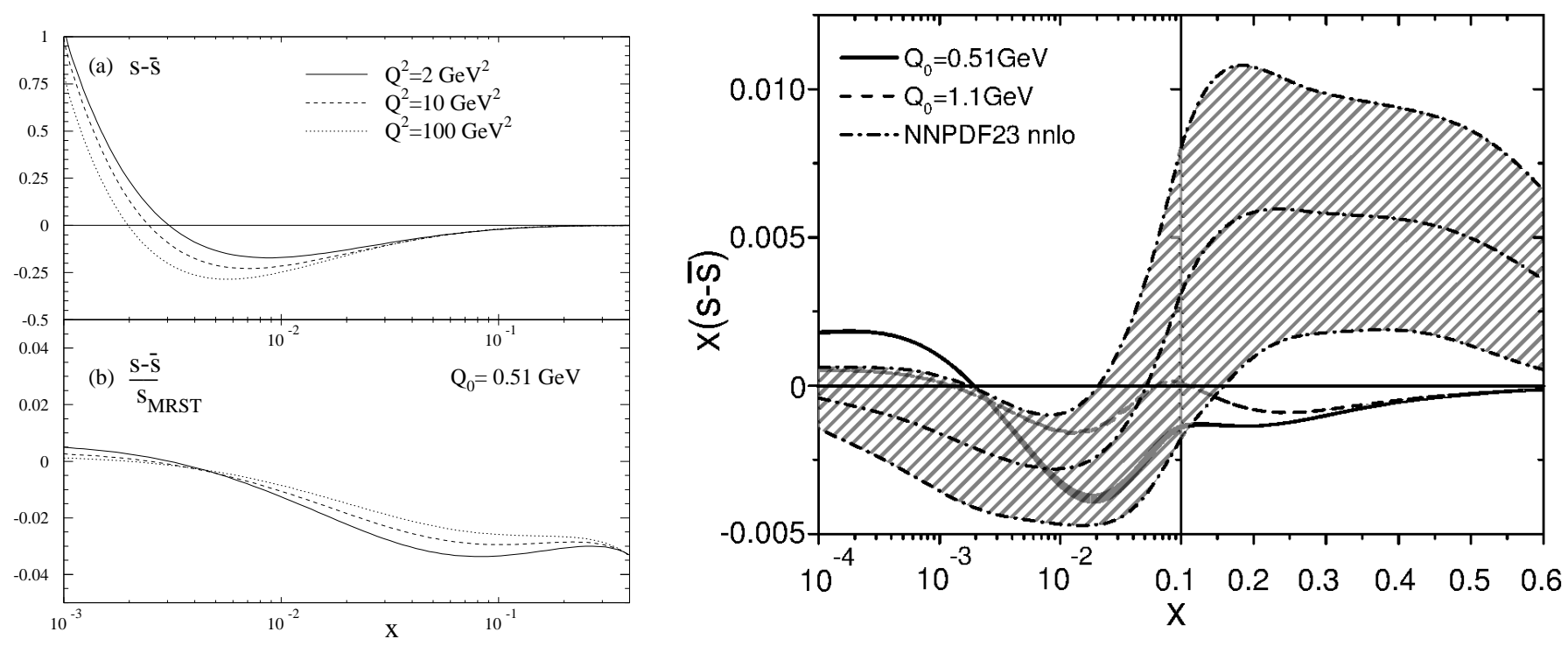

Figure 15: Left: (a) The asymmetry $s(x)-\bar{s}(x)$ from perturbative NNLO QCD calculation (b) the ratio to the LO strange density. Figure from [90. Right: (c) The perturbative strangeness asymmetry $x(s(x)-\bar{s}(x))$ at $Q^{2}=16 \mathrm{GeV}^{2}$ with the initial scale $Q_{0}=0.51 \mathrm{GeV}$ (solid line) and $1.1 \mathrm{GeV}$ (deashed line). The small bands represent the $1 \sigma$ range due to the uncertainties associated with the $d_{v}$ and $u_{v}$ of ABM11 PDF. The total asymmetry from NNLO NNPDF2.3 PDF and its $1 \sigma$ uncertainty band is overlaid. Figure from [95].

The nonperturbative contributions of strangeness asymmetry could come from nucleon fluctuating 
into virtual hyperon and kaon pairs such as $\Lambda K$ and $\Sigma K$. This mechanism of kaon cloud is very similar to that of non-strange meson cloud which leads to the $\bar{d} / \bar{u}$ asymmetry. Nevertheless theoretical predictions of $s(x)-\bar{s}(x)$ are rather diverse. Signal and Thomas [91] predicted that the $s$ and $\bar{s}$ have different distributions with the bag model and the quantitative results depended on the bag radius. Holtmann et al. 92] found that $s<\bar{s}$ in small $x$ region and $s>\bar{s}$ in large $x$ region using the meson cloud model with the fluctuation function calculated from time-ordered perturbative theory in the infinite momentum frame. However Brodsky and Ma [93] reached an opposite conclusion using a lightcone two-body wave function model for the description of the meson-baryon fluctuation. It was shown in Ref. 94 that the difference between $s(x)$ and $\bar{s}(x)$ is sensitive to the splitting functions of hyperon and kaon as well as the distributions of strange partons inside the virtual hadrons.

In the chiral quark model [35, 36] and the chiral-quark soliton model [38, 39], the coupling of meson cloud is via the constituent quark of the nucleon and $s(x)>\bar{s}(x)$ in large $x$ region is predicted. In a recent study of strangeness asymmetry [95, both nonperturbative meson cloud and perturbative NNLO contributions are taken into account simultaneously. It is found that the nonperturbative contribution dominates in the region of $x \geq 0.1$ while the perturbative ones are more significant in the smaller $x$ region, as shown in Fig. 15 (c), compared with the results of NNPDF global analysis [18]. The best region to detect this asymmetry experimentally is claimed to be $0.02<x<0.03$ and more than one node of the $s(x)-\bar{s}(x)$ asymmetry is suggested.

\subsubsection{Strange quarks from the global analysis}

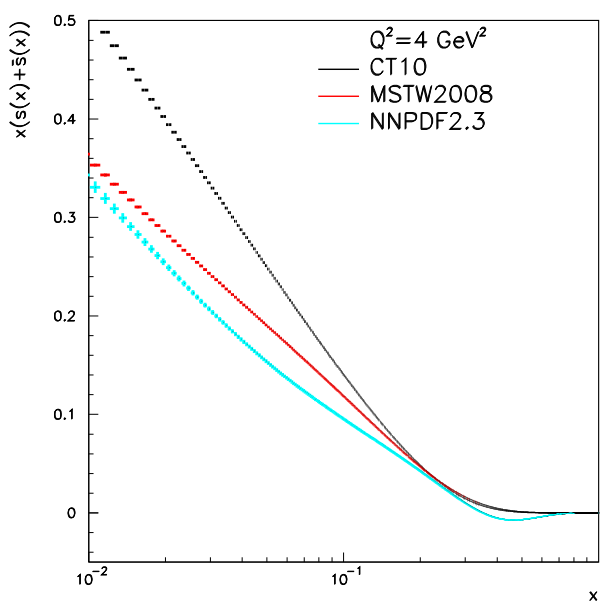

(a)

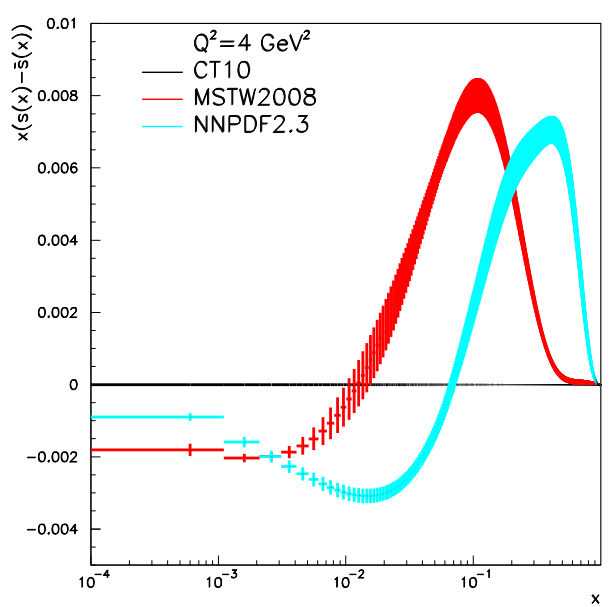

(b)

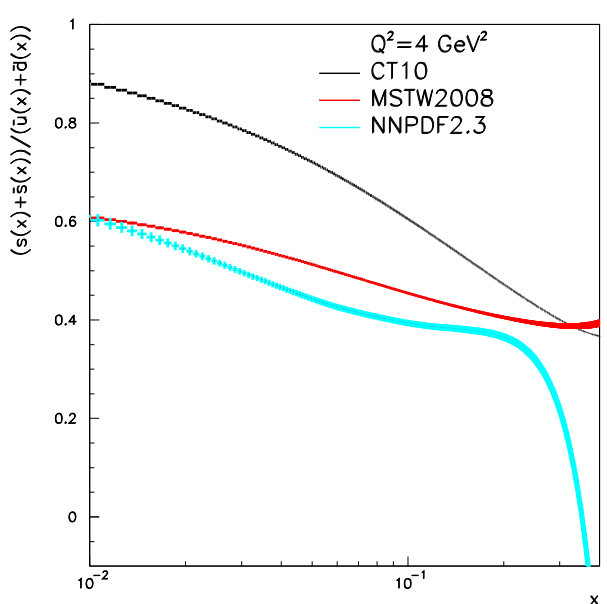

(c)

Figure 16: The distributions of $x(s(x)+\bar{s}(x)), x(s(x)-\bar{s}(x))$ and $(s(x)+\bar{s}(x)) /(\bar{u}(x)+\bar{d}(x))$ from CT10 [16], MSTW2008 [17] and NNPDF2.3 [18] PDFs at $Q^{2}=4 \mathrm{GeV}^{2}$.

Figure 16 shows the distributions of $x(s(x)+\bar{s}(x)), x(s(x)-\bar{s}(x))$ and $(s(x)+\bar{s}(x)) /(\bar{u}(x)+\bar{d}(x))$ at $Q^{2}=4 \mathrm{GeV}^{2}$ from CT10 [16], MSTW2008 [17] and NNPDF2.3 [18]. There is an assumption of $s$ and $\bar{s}$ symmetry in CT10 while $s-\bar{s}$ asymmetry is allowed in MSTW2008 and NNPDF2.3. For MSTW2008 and NNPDF2.3, the strange quark sea $s+\bar{s}$ is suppressed relative to the non-strange sea $\bar{u}+\bar{d}$ over the whole $x$ region. The strange quarks in CT10 are less suppressed than that in MSTW2008 and NNPDF2.3.

It was noted that the dimuon data from neutrino scattering and improved analyses are important to constrain the uncertainty of strange parton distributions in the global analyses [76]. The inclusion of measurements of $W$ and $Z$ bosons and inclusive jet production at LHC should further improve the 
constraints at the small $x$ region [18, 76]. Very recently a new determination of nucleon strange sea was obtained using data including neutrino DIS, lepton DIS and $W$ production at LHC [96]. In this study it is found that the $x$ dependence of the strange sea distribution is similar to the non-strange one. Figure $17(\mathrm{a})$ shows two $x$ distributions of $r_{s}$ at the energy scale $\mu^{2}=1.9 \mathrm{GeV}^{2}$ which are obtained by the combination of NuTeV/CCFR [68], CHORUS [73] and NOMAD [67], and the combination of CHORUS [73], CMS [82] and ATLAS [80], respectively. This new determination is consistent with the results obtained by CMS [82] from the analysis of the $W+c$ production in combination with the HERA DIS data [81]. However, a much higher value of $r_{s} \sim 1$ was obtained by ATLAS [80] from the fit of the ATLAS data on the $W, Z$ production [79] together with the HERA DIS data.

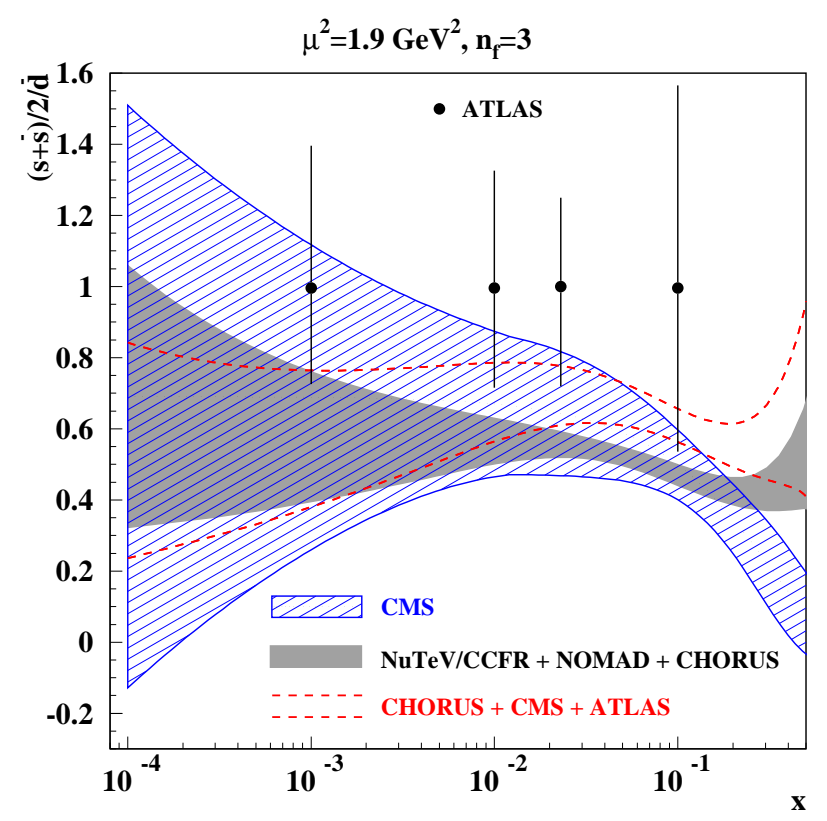

(a)

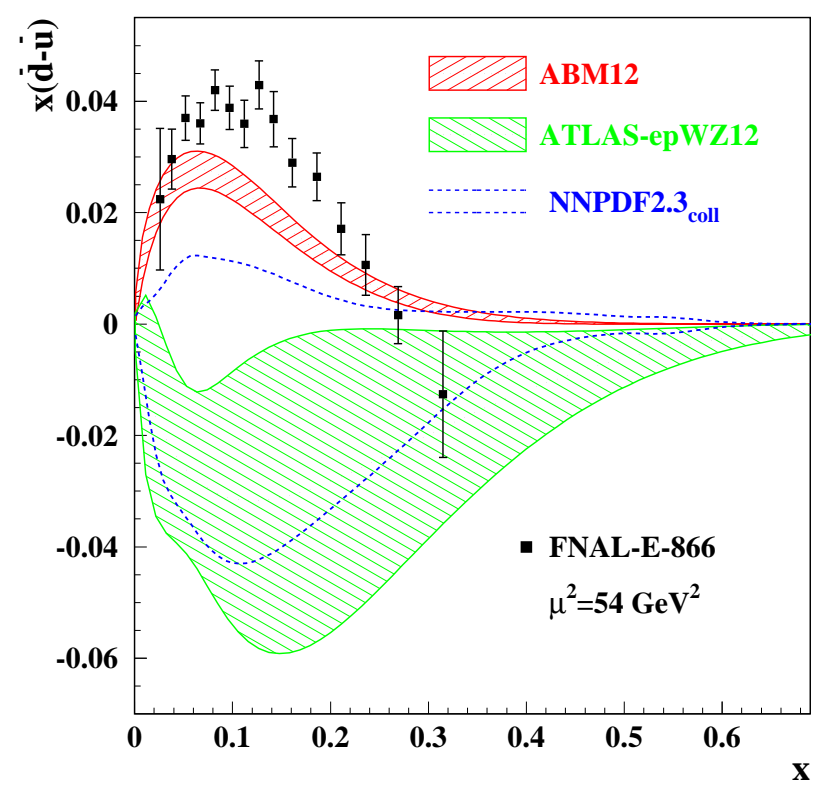

(b)

Figure 17: (a) The strange sea suppression factor $r_{s}=(s+\bar{s}) / 2 \bar{d}$ and uncertainty band as a function of $x$ obtained in the analyses based on the combination of the data by NuTeV/CCFR [68] CHORUS [73], and NOMAD [67] (shaded area) and CHORUS [73], CMS [82], and ATLAS [80] (dashed lines), in comparison with the results obtained by the CMS analysis [82] (hatched area) and by the ATLAS epWZ-fit [79, 80] (full circles). The energy scale $\mu^{2}$ is $1.9 \mathrm{GeV}^{2}$. (b) The asymmetry of the non-strange sea $x(\bar{d}-\bar{u})$ at the scale of $\mu^{2}=54 \mathrm{GeV}^{2}$ as a function of $x$ obtained in the ABM12 fit [96] (right-tilted hatch), in comparison with the corresponding ones obtained by the ATLAS [79] (left-tilted hatch) and the NNPDF [18] (dashed lines) analyses using only the LHC and HERA collider data. Data of $x(\bar{d}-\bar{u})$ shown as full squares are from the FNAL E866 Drell-Yan experiment [23]. Figures from [96].

This discrepancy is investigated and the speculation is that there is a lack of sufficient constraints of non-strange sea from solely HERA DIS data and ATLAS data. Figure 17(b) shows the $x$ distributions of non-strange sea asymmetry $x(\bar{d}(x)-\bar{u}(x))$ from several global analyses at $\mu^{2}=54 \mathrm{GeV}^{2}$, compared to the FNAL E866 Drell-Yan data [23. Obviously the value of $x(\bar{d}-\bar{u})$ obtained in ATLAS analysis [80] is negative and that from the analysis with only the LHC and HERA collider data in NNPDF2.3 is also slightly negative, in disagreement with the E866 Drell-Yan data. This finding suggests that the strange sea enhancement observed in the analysis of ATLAS [79, 80] might be achieved at the expense of a suppressed $\bar{d}$ distribution because the HERA inclusive DIS data do not have enough sensitivity to the flavor structure of antiquarks. 


\subsection{Five-quark intrinsic sea model and lattice QCD}

Below we will review some recent progress in the theoretical interpretations with regard to the observed breaking of $\mathrm{SU}(2)$ and $\mathrm{SU}(3)$ flavor symmetry of light quark sea in terms of the five-quark intrinsic sea and lattice QCD.

\subsubsection{Intrinsic and extrinsic sea}

The possible existence of a significant $|u u d c \bar{c}\rangle$ five-quark Fock component in the proton was proposed long time ago by Brodsky, Hoyer, Peterson, and Sakai (BHPS) [97] to explain the unexpectedly large production rates of charmed hadrons at large forward $x_{F}$ region. The intrinsic charm originating from the five-quark Fock state is to be distinguished from the "extrinsic" charm produced in the splitting of gluons into $c \bar{c}$ pairs. Typical diagrams of gluon splitting, gluon fusion and light quark scattering characterizing the extrinsic and intrinsic sea [98] are shown in Fig. 18. The extrinsic charm has a "sealike" characteristics with large magnitude only at the small $x$ region. In contrast, the intrinsic charm is "valence-like" with a distribution peaking at larger $x$.

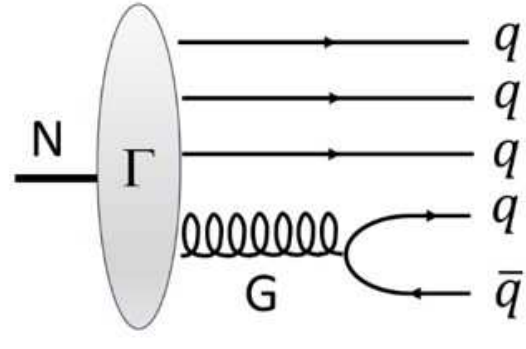

(a)

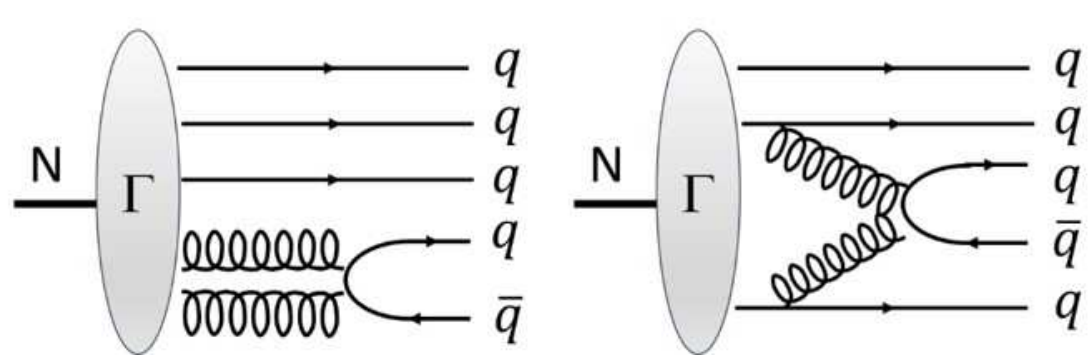

(b)

(c)

Figure 18: (a) Gluon splitting contributing to the extrinsic sea quarks in the proton wave function 988. (b) Gluon fusion and (c) light quark scattering contributing to the intrinsic sea quarks in the proton wave function 98 .

Since the BHPS model predicts the probability for the $|u u d Q \bar{Q}\rangle$ configuration to be inversely proportional to $m_{Q}^{2}$, where $m_{Q}$ is the mass of the quark $Q$ [97], the light quark sector could in principle provide more clear evidences for the roles of the five-quark Fock states, allowing the specific predictions of the BHPS, such as the shape of the quark momentum distributions originating from the five-quark configuration, to be tested. To search for evidence for the intrinsic five-quark Fock states, it is essential to separate the contributions of the intrinsic sea from those of the extrinsic one.

In Ref. [99, 100, 101], the BHPS model was extended to the light quark sector and the predictions were compared with the experimental data of $\bar{d}-\bar{u}, s+\bar{s}$, and $\bar{u}+\bar{d}-s-\bar{s}$ from E866 [23], HERMES [74] experiments and the PDF set CTEQ6.6 [102]. The $\bar{d}-\bar{u}$ and $\bar{u}+\bar{d}-s-\bar{s}$ are the $\mathrm{SU}(2)$ and SU(3) flavornonsinglet quantities which are free from the contributions of the extrinsic sea quarks. Non-perturbative effects could lead to different probabilities for the $|u u d d \bar{d}\rangle,|u u d u \bar{u}\rangle$ and $|u u d s \bar{s}\rangle$ configurations. The $x$ distributions of the $\bar{q}$ sea in $|u u d q \bar{q}\rangle$ were first constructed in the BHPS model at a certain initial scale $\mu$ and then evolved to the $Q^{2}$ of the experiments to compare with the data. As for the HERMES $s+\bar{s}$ data, the sharp rise towards small $x$ is consistent with the extrinsic sea, while the data at $x>0.1$ is interpreted as the intrinsic component of strange sea, as described by the BHPS model. 


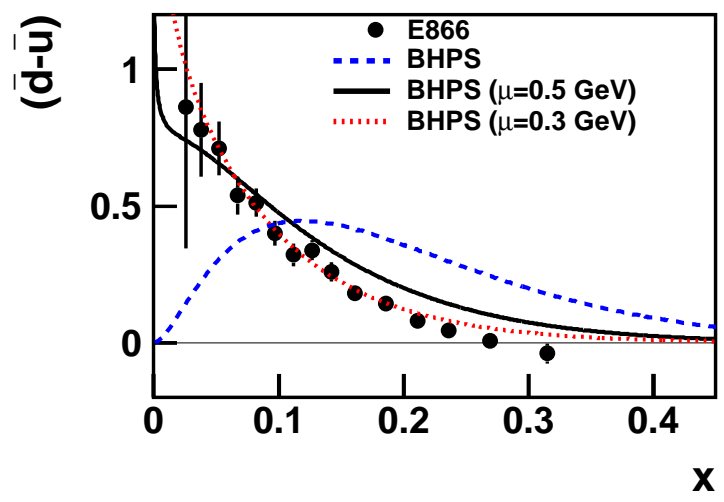

(a)

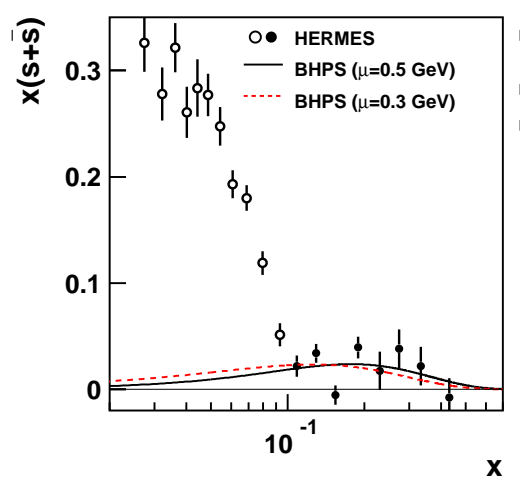

(b)

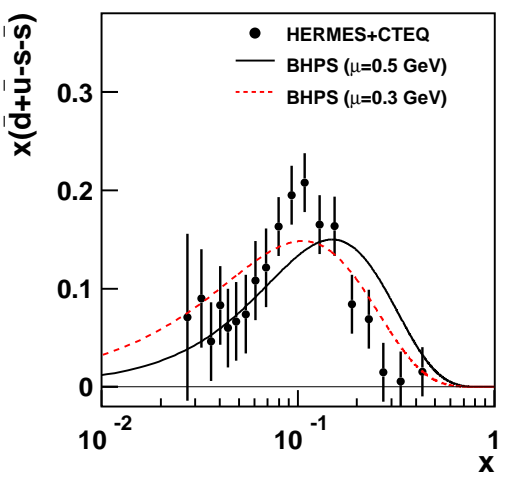

(c)

Figure 19: (a) Comparison of the $\bar{d}(x)-\bar{u}(x)$ data with the calculations based on the BHPS model. The blue dashed curve corresponds to the BHPS result at initial scale, and the solid and dotted curves are obtained by evolving the BHPS result to $Q^{2}=54.0 \mathrm{GeV}^{2}$ using $\mu=0.5 \mathrm{GeV}$ and $\mu=0.3 \mathrm{GeV}$, respectively. (b) Comparison of the HERMES $x(s(x)+\bar{s}(x))$ data with the calculations based on the BHPS model. The solid and dashed curves are obtained by evolving the BHPS result to $Q^{2}=2.5 \mathrm{GeV}^{2}$ using $\mu=0.5 \mathrm{GeV}$ and $\mu=0.3 \mathrm{GeV}$, respectively. The normalizations of the calculations are adjusted to fit the data at $x>0.1$. (c) Comparison of the $x(\bar{d}(x)+\bar{u}(x)-s(x)-\bar{s}(x))$ data with the calculations based on the BHPS model. The values of $x(s(x)+\bar{s}(x))$ are from the HERMES experiment [74, and those of $x(\bar{d}(x)+\bar{u}(x))$ are obtained from the PDF set CTEQ6.6 102. The solid and dashed curves are obtained by evolving the BHPS result to $Q^{2}=2.5 \mathrm{GeV}^{2}$ using $\mu=0.5 \mathrm{GeV}$ and $\mu=0.3 \mathrm{GeV}$, respectively. The normalization of the calculations are adjusted to fit the data.

The good agreement between the theory and the data shown in Fig. 19(a), 19(b), and 19(c), is interpreted as evidence for the existence of the intrinsic light-quark sea in the nucleons. The probabilities for the $|u u d u \bar{u}\rangle,|u u d d \bar{d}\rangle$ and $|u u d s \bar{s}\rangle$ Fock states are also extracted as : $\mathcal{P}_{5}^{u \bar{u}}=0.122, \mathcal{P}_{5}^{d \bar{d}}=0.240$ and $\mathcal{P}_{5}^{s \bar{s}}=0.024$ for the initial scale $\mu=0.5 \mathrm{GeV}$, or $\mathcal{P}_{5}^{u \bar{u}}=0.162, \mathcal{P}_{5}^{d \bar{d}}=0.280$ and $\mathcal{P}_{5}^{s \bar{s}}=0.029$ for $\mu=0.3 \mathrm{GeV}$. The results agree reasonably well with $\mathcal{P}_{5}^{u \bar{u}}=0.098, \mathcal{P}_{5}^{d \bar{d}}=0.216$ and $\mathcal{P}_{5}^{s \bar{s}}=0.057$ evaluated by the extended chiral constituent quark model [103].

\subsubsection{Connected and disconnected sea}

According to the Euclidean path-integral formalism of the hadronic tensor, there are two distinct sources for the nucleon sea: the connected sea (CS) and the disconnected sea (DS). The existence of the connected sea and disconnected sea can be illustrated in three gauge invariant and topologically distinct diagrams, as shown in Fig. 20. The various lines in Fig. 20 represent the quark propagators from the source of the nucleon interpolation field at time $t=0$ to the sink time at $t$ and the currents are inserted at $t_{1}$ and $t_{2}$. These two components are expected to have different $x$ distributions, as well as quark-flavor dependence. Since there exists $u$ and $d$ valence quarks in the nucleons, the $u$ and $d$ have both the CS and DS while $s$ and $c$ have only the DS. The small- $x$ behavior is scaled as $x^{-1 / 2}$ for the valence and CS, and $x^{-1}$ for the DS. 


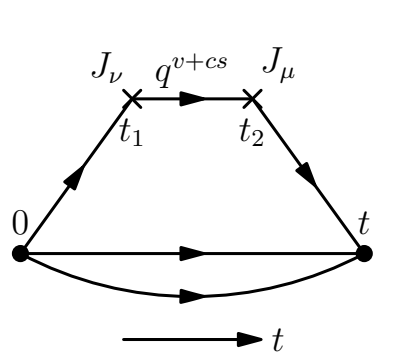

(a)

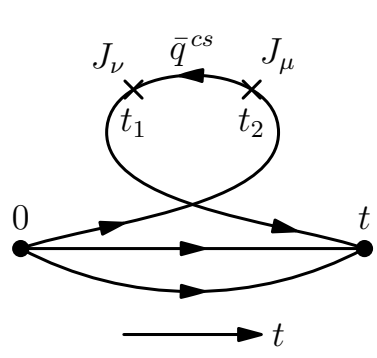

(b)

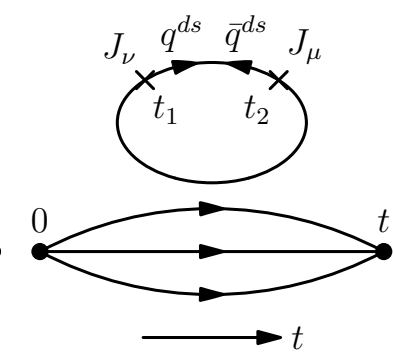

(c)

Figure 20: Three gauge invariant and topologically distinct diagrams in the Euclidean path-integral formalism of the nucleon hadronic tensor in the large momentum frame. In between the currents at $t_{1}$ and $t_{2}$, the parton degrees of freedom are (a) the valence and CS partons $q^{v+c s}$, (b) the CS antipartons $\bar{q}^{c s}$, and (c) the DS partons $q^{d s}$ and antipartons $\bar{q}^{d s}$ with $q=u, d, s$, and $c$. Only $u$ and $d$ are present in (a) and (b).

It is clear from Fig. 20 that the two sources of the sea quarks, CS and DS, have interesting quarkflavor dependence. For example, while $u$ and $d$ have both CS and DS, $s$ and $c$ have only DS. The small mass difference between the $u$ and $d$ quarks implies that the DS cannot account for the large $\bar{d} / \bar{u}$ difference. Accordingly the flavor-nonsinglet quantity $x(\bar{d}(x)-\bar{u}(x))$ is mainly contributed by the CS component. A lattice calculation of the ratio $R$ of momentum fraction $\langle x\rangle$ for the strange and $u(d)$ in the disconnected insertion moment is found to be 0.857(40) [104. This result is utilized to derive the DS component of $x(\bar{u}(x)+\bar{d}(x))$ as $\frac{1}{R} x(s(x)+\bar{s}(x))$ from HERMES's results of $x(s(x)+\bar{s}(x))$ [105].

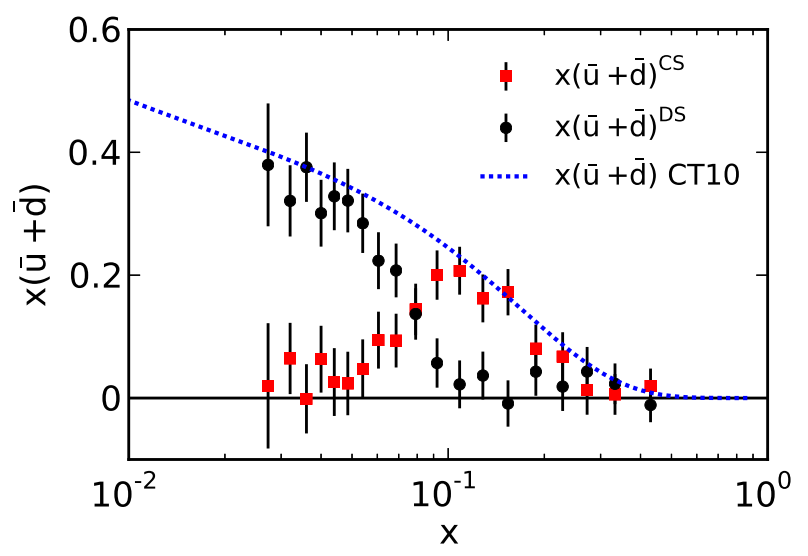

(a)

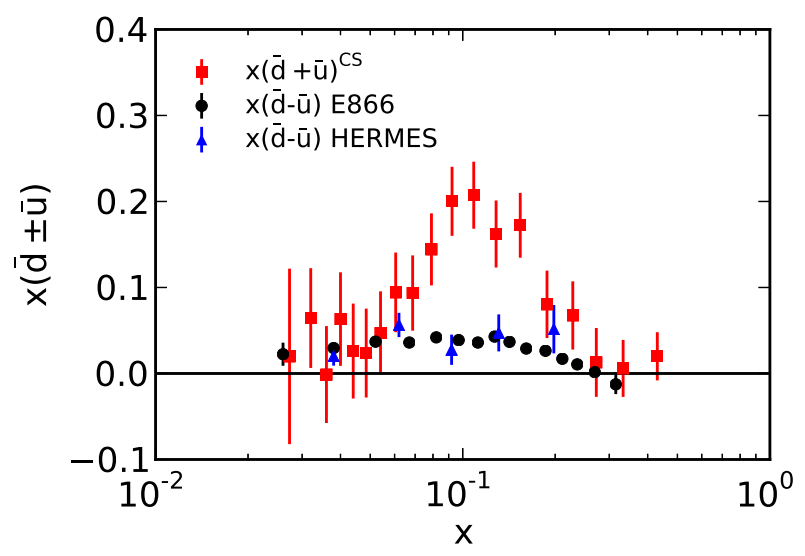

(b)

Figure 21: $x\left(\bar{u}^{c s}(x)+\bar{d}^{c s}(x)\right)$ plotted together with (a) $x(\bar{u}(x)+\bar{d}(x))$ from CT10 and $\frac{1}{R} x(s(x)+\bar{s}(x))$ which is taken to be $x\left(\bar{u}^{d s}(x)+\bar{d}^{d s}(x)\right)$ and (b) $x(\bar{d}(x)-\bar{u}(x))$ from E866 Drell-Yan experiment [23] and from SIDIS HERMES experiment [24]

The CS component of $u, d$ sea could be obtained by the subtraction of the derived DS component from the CT10 [16] results of $u, d$ sea as shown in Fig. 21(a), In Fig. 21(b), the derived CS component of $u, d$ sea is plotted together with two data sets of $x(\bar{d}(x)-\bar{u}(x))$ from E866 at $Q^{2}=54 \mathrm{GeV}^{2}$ and from HERMES at $<Q^{2}>=2.3 \mathrm{GeV}^{2}$. The valence-like shape of these two distributions lends support to the lattice interpretation on the origins of sea quarks. Since CS evolves like the valence, it is suggested to have the CS and DS separately accommodated in the extended QCD evolution to facilitate the global fitting 105]. 


\subsubsection{Comparison between two approaches}

The above two approaches take apparently different views of the origin of sea quarks in interpreting the same data set and it is interesting to compare them. The large $\bar{d} / \bar{u}$ difference observed in the DIS and Drell-Yan experiments is considered to originate primarily from the CS or intrinsic component of sea quark, both of which are of non-perturbative nature. The structure of $x$ distribution is viewed as composed of both extrinsic (at small- $x$ ) and intrinsic (at large- $x$ ) components. The CS component is mostly associated with the intrinsic sea while the DS component contains both intrinsic and extrinsic sea.

\subsection{Heavy Quarks Sea}

The existence of heavy quarks sea in the PDF of the proton at initial scale other than those arising perturbatively through gluon splitting in the DGLAP evolution, is a long standing issue in high-energy physics. The charm structure functions $F_{2 c}^{\gamma p}$ in the relatively large- $x$ region measured by the EMC collaboration [106, 107, 108, 109, 110] were inconsistent with the sole production of charm by photongluon fusion [29]. This brought the speculation about the charm of nonperturbative origin, so-called "intrinsic charm" (IC). Two nonperturbative models of charm are suggested, the light-cone five-quark BHPS model [29] and the virtual meson cloud model for the proton wave function [111, 112].

This question is addressed quantitatively by the CTEQ Collaboration by examining all relevant hard-scattering data in the global analysis of CTEQ6.5c [113]. Three types of parametrization of charm quarks originating from various scenarios were used at the initial scales:

- (a) the light-front five-quark BHPS model

$$
c(x)=\bar{c}(x)=A x^{2}\left[6 x(1+x) \ln x+(1-x)\left(1+10 x+x^{2}\right)\right]
$$

- (b) virtual meson-cloud model

$$
c(x)=A x^{1.897}(1-x)^{6.095} \text { and, } \bar{c}(x)=\bar{A} x^{2.511}(1-x)^{4.929}
$$

and

- (c) perturbatively sea-like, i.e. similar to that of the light quarks sea.

$$
c(x)=\bar{c}(x)=A[\bar{u}(x)+\bar{d}(x)]
$$

where $A$ and $\bar{A}$ are normalization constants. The goodness-of-fit in the global analysis shows improvement with the inclusion of the additional charm component at the initial scale. 

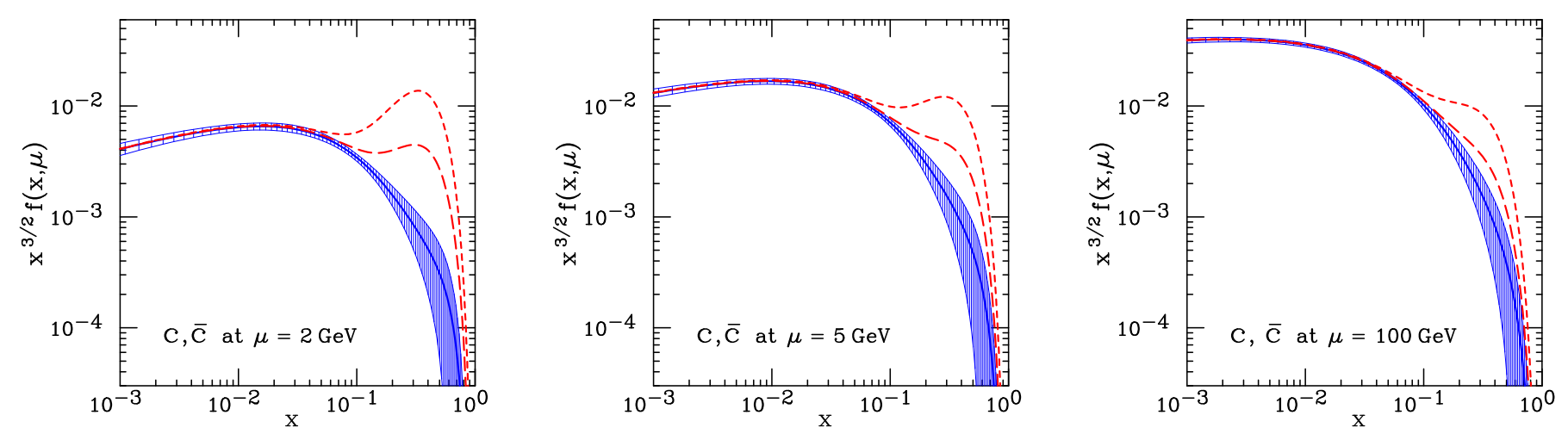

Figure 22: Charm quark distributions from the BHPS IC model. The three panels correspond to scales $\mu=2, \mu=5$, and $\mu=100 \mathrm{GeV}$. The long-dash (short-dash) curve corresponds to $\langle x\rangle_{c+\bar{c}}=0.57 \%$ (2.0\%). The solid curve and shaded region show the central value and uncertainty from CTEQ6.5, which contains no IC. Figures from [113].
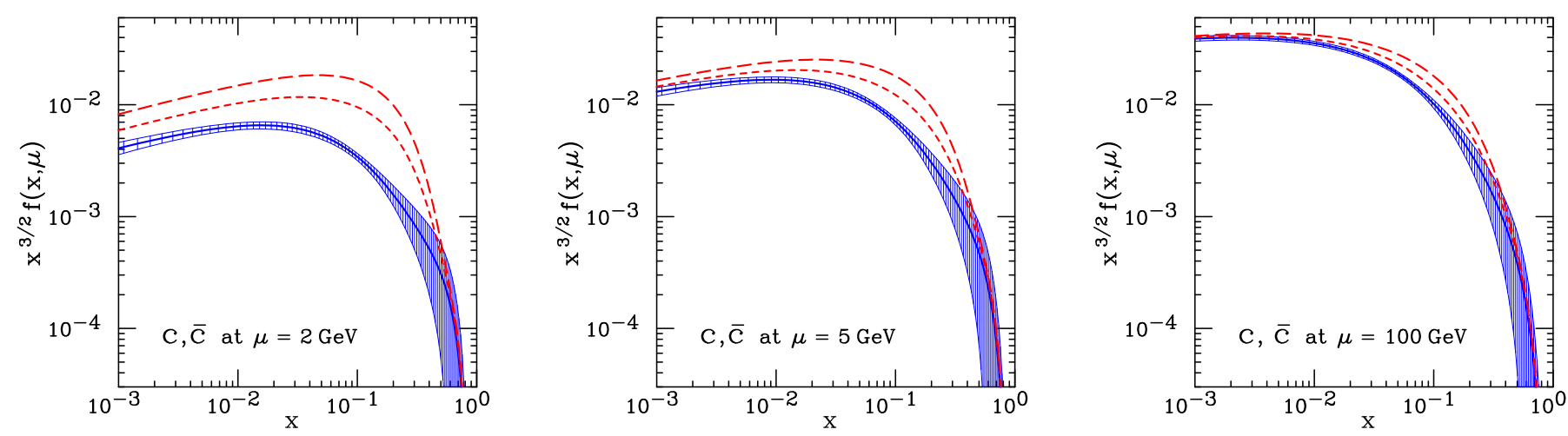

Figure 23: Same as Fig.22, except for the sea-like scenario. The long-dash (short-dash) curves correspond to $\langle x\rangle_{c+\bar{c}}=2.4 \%$ (1.1\%). Figures from [113].

The magnitude of intrinsic charm is characterized by the momentum fraction $\langle x\rangle_{c+\bar{c}}\left(\equiv \int_{0}^{1} x[c(x)+\right.$ $\bar{c}(x)] d x$ ) carried by charm at the initial scale $\mu=1.3 \mathrm{GeV}$. For the shape suggested by non-perturbative light-cone BHPS model, the data are consistent with a wide range of the intrinsic charm magnitude, ranging from null to 2-3 times larger than the estimate by the BHPS model as shown in Fig. 22. The marginally allowed amount of $\mathrm{IC}$ is $\langle x\rangle_{c+\bar{c}}=0.020$. A salient feature is that there could be a large enhancement of charm at $x>0.1$ relative to the PDF assuming no IC even at a scale as large as $\mu=$ $100 \mathrm{GeV}$. Fig. 23 shows the result for an assumed shape of IC similar to other sea quarks; the maximum amount of IC is $\langle x\rangle_{c+\bar{c}}=0.024$ and the enhancement of charm shows up in a more broad range of $x$, $0.01<x<0.50$.

Very recently, CTEQ-TEA group reported an updated study of IC in the structure function of the proton with CT10 NNLO global analysis [114]. Besides the advances in the theoretical approach, more relevant data set is included: the combined H1 and ZEUS data for DIS [115] and inclusive charm production [116] new data set are found to constrain the allowance of the sea-like IC more strongly than other data. Figure 24 shows the charm parton density distribution in the new global fits and the conclusion is that a reasonable global analysis could still be done without an IC component of proton but a small IC component with $\langle x\rangle_{I C} \leq 2.5 \%$ for valence-like BHPS IC or $\langle x\rangle_{I C} \leq 1.5 \%$ for sea-like IC cannot be ruled out. Again, this calls for more relevant data to pin down the existence of IC. 

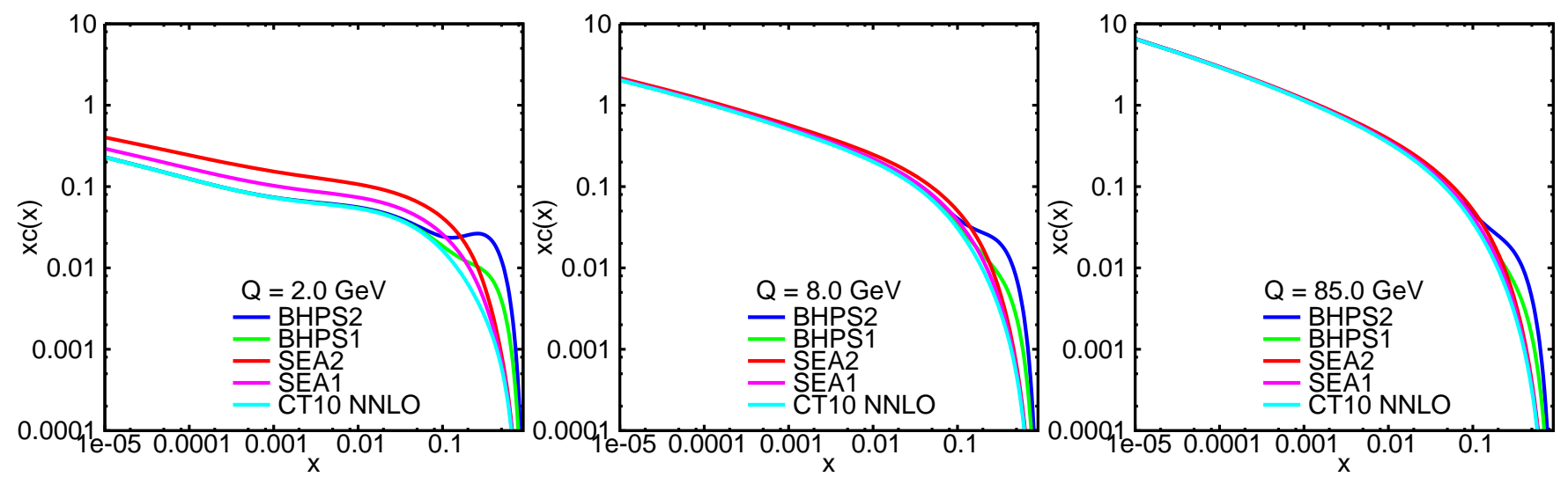

Figure 24: Charm quark distribution $x c(x, Q)$ from the BHPS1 and BHPS2 PDFs (which have 0.57\% and $2 \%\langle x\rangle_{\mathrm{IC}}$ ); from SEA1 and SEA2 PDFs (which have $0.57 \%$ and $1.5 \%\langle x\rangle_{\mathrm{IC}}$ ); and from CT10. The corresponding energy scale $Q$ of these three graphs is 2.0, 8.0, and $85 \mathrm{GeV}$ respectively. Figures from [114.

\section{Polarized Distributions of Sea Quarks}

There are several important motivations for measuring and understanding the helicity distributions of sea quarks in the nucleons. First, the discovery of the spin puzzle at SLAC and NMC showed clearly that the expectation from naive $\mathrm{SU}(3)$ that quarks provide essentially all the spin of the nucleon is invalid. This led to extensive theoretical and experimental efforts during the last several decades to understand the decomposition of the proton's spin in terms of the quark/antiquark spin, gluon spin, and quark/gluon orbital angular momenta. While the recent polarized semi-inclusive DIS and $W$ production in polarized $p p$ collision suggest a relatively small contribution of sea quarks to the proton's spin, the $x$ and flavor dependence of the antiquark helicity distributions are still poorly known. Second, as discussed below, many theoretical models which are capable of explaining the flavor structure of the unpolarized nucleon sea also have specific predictions on the helicity distributions of the nucleon sea. While these models have very similar predictions for the unpolarized sea, they often differ significantly on their predictions of the polarized sea. Therefore, a stringent test of the various theoretical models could be provided by more precise information on the sea-quark polarizations. Finally, the advent of the lattice QCD now offers the possibility to calculate the $x$ distributions of the sea-quark polarizations, and these calculations could be tested against the experimental data in the future.

In this Section we first discuss the subject of the helicity distributions of the light-quark sea, namely $\Delta \bar{u}$ and $\Delta \bar{d}$. We then examine the current knowledge on the polarization of strange-quark sea, followed by a summary of future prospects.

\subsection{Asymmetry Between $\Delta \bar{u}(x)$ and $\Delta \bar{d}(x)$}

The surprisingly large flavor asymmetry between the $\bar{u}(x)$ and $\bar{d}(x)$ naturally leads to the question whether the polarized $\bar{u}$ and $\bar{d}$ seas are also asymmetric. The flavor and the spin structures of the nucleon sea are closely connected in the sense that many theoretical models, originally proposed to explain the $\bar{d} / \bar{u}$ flavor asymmetry, also have specific implications for the spin structure of the nucleon sea. We now briefly summarize the predictions from various theoretical models on the flavor asymmetry of the light-quark helicity distributions. 


\subsubsection{Theoretical predictions on the asymmetry between $\Delta \bar{u}(x)$ and $\Delta \bar{d}(x)$}

- In the pion-cloud model, a pion is emitted from the proton in a p-wave state to conserve parity. This reduces the spin projection of the baryons $(N$ or $\Delta)$ in the $\pi-N$ or $\pi-\Delta$ configurations along the initial proton spin direction. Therefore, pion cloud would lead to a reduction of the proton's spin residing in the quark's spin in qualitative agreement with the experimental results. This implies that a fraction of the proton's spin would reside in the orbital angular momentum of the pion-baryon configuration. Moreover, the antiquarks $(\bar{u}$ and $\bar{d})$ in the pion-baryon configurations are unpolarized due to the spin-0 nature of pion. Therefore, the pion-cloud model would predict that $\Delta \bar{u}=\Delta \bar{d}=0$. An extension of the meson cloud model to include the kaon-hyperon configuration would lead to the conclusion that $\bar{s}$ quarks, which are present in the spin-0 kaon cloud, are unpolarized. On the other hand, the strange quarks $(s)$ residing in the hyperons would be polarized. By extending the meson cloud model to include a vector meson $(\rho)$ cloud, non-zero $\bar{u}, \bar{d}$ sea quark polarizations with $\Delta \bar{d}-\Delta \bar{u}>0$ were predicted [117, 118, 119, 120].

- In the chiral-quark model, a quark would undergo a spin flip upon an emission of a pseudoscalar meson $\left(u^{\uparrow} \rightarrow \pi^{\circ}(u \bar{u}, d \bar{d})+u^{\downarrow}, u^{\uparrow} \rightarrow \pi^{+}(u \bar{d})+d^{\downarrow}, u^{\uparrow} \rightarrow K^{+}+s^{\downarrow}\right.$, etc.). This model predicts that antiquarks $(\bar{u}, \bar{d}, \bar{s})$ are unpolarized $(\Delta \bar{u}=\Delta \bar{d}=\Delta \bar{s}=0)$ since they only reside in spin-0 Goldstone bosons. In contrast, the strange quarks $(s)$ would have a negative polarization since the $u$ valence quarks in the proton are positively polarized and the $u^{\uparrow} \rightarrow K^{+}+s^{\downarrow}$ process would lead to an excess of $s^{\downarrow}$.

- The Pauli-blocking model [121] implies that an excess of $q^{\uparrow}\left(q^{\downarrow}\right)$ valence quarks would inhibit the creation of a pair of $q^{\uparrow} \bar{q}^{\downarrow}\left(q^{\downarrow} \bar{q}^{\uparrow}\right)$ sea quarks. Since the polarization of the $u(d)$ valence quarks in the proton is positive (negative), the Pauli-blocking model predicts a positive (negative) polarization for the $\bar{u}(\bar{d})$ sea $(\Delta \bar{u}>0>\Delta \bar{d})$.

- In the instanton model [122], scattering of a valence quark off a nonperturbative fluctuation of the gluon field, i.e. instanton, can result in a quark-antiquark pair. The instanton-induced interaction is described by the 't Hooft effective Lagrangian, which allows processes such as $u^{\uparrow} \rightarrow u^{\downarrow} d^{\uparrow} \bar{d}^{\downarrow}$, $d^{\downarrow} \rightarrow d^{\uparrow} u^{\downarrow} \bar{u}^{\uparrow}$. Since the flavor of the quark-antiquark pair generated in this process is different from that of the initial valence quark, the instanton model readily explains $\bar{d}>\bar{u}$. Furthermore, the correlation between the helicities of the sea quark and the valence quark in the effective Lagrangian (i.e. $u^{\uparrow}$ leads to a $\bar{d} \downarrow$ ) predicts a positively (negatively) polarized $\bar{u}(\bar{d})$ sea. In particular, it predicts a large $\Delta \bar{u}, \Delta \bar{d}$ flavor asymmetry with $\Delta \bar{u}>\Delta \bar{d}$, namely, $\int_{0}^{1}[\Delta \bar{u}(x)-\Delta \bar{d}(x)] d x=\frac{5}{3} \int_{0}^{1}[\bar{d}(x)-$ $\bar{u}(x)] d x[123]$.

- In the chiral-quark soliton model [124, 125, QCD at the large $N_{c}$ limit becomes an effective theory of mesons with the baryons appearing as solitons. Quarks are described by single particle wave functions, which are solutions of the Dirac equation in the field of the background pions. In this model, the unpolarized isovector distributions $\bar{u}(x)-\bar{d}(x)$ appear in next-to-leading order $\left(N_{c}\right)$ in a $1 / N_{c}$ expansion, while the polarized isovector distributions $\Delta \bar{u}(x)-\Delta \bar{d}(x)$ appears in leading-order $\left(N_{c}^{2}\right)$. Therefore, this model predicts a large flavor asymmetry for the polarized sea $[\Delta \bar{u}(x)-\Delta \bar{d}(x)]>[\bar{d}(x)-\bar{u}(x)]$.

- In the statistical model [41, 126], the nucleon is considered as a collection of massless quarks, antiquarks, and gluons in thermal equilibrium within a finite volume. The momentum distributions for quarks and antiquarks follow a Fermi-Dirac function characterized by a common temperature and a chemical potential $\mu$ which depends on the flavor and helicity of the quarks. It can be shown that

$$
\mu_{\bar{q} \uparrow}=-\mu_{q \downarrow} ; \mu_{\bar{q} \downarrow}=-\mu_{q \uparrow} .
$$


Table 5: Prediction of various theoretical models on the integral $I_{\Delta}=\int_{0}^{1}[\Delta \bar{u}(x)-\Delta \bar{d}(x)] d x$.

\begin{tabular}{lll}
\hline Model & $I_{\Delta}$ prediction & Ref. \\
\hline Meson cloud $(\pi$-meson) & 0 & {$[31,[127]$} \\
Meson cloud $(\rho$-meson) & $\simeq-0.0007$ to -0.027 & {$[117]$} \\
Meson cloud $(\pi-\rho$ interf.) & $=-6 \int_{0}^{1} g^{p}(x) d x$ & {$[118]$} \\
Meson cloud $(\rho$ and $\pi-\rho$ interf. $)$ & $\simeq-0.004$ to -0.033 & {$[119]$} \\
Meson cloud $(\rho$-meson) & $<0$ & {$[120]$} \\
Meson cloud $(\pi-\sigma$ interf. $)$ & $\simeq 0.12$ & {$[132]$} \\
Pauli-blocking (bag-model) & $\simeq 0.09$ & {$[119]$} \\
Pauli-blocking (ansatz) & $\simeq 0.3$ & {$[128]$} \\
Pauli-blocking & $=\frac{5}{3} \int_{0}^{1}[\bar{d}(x)-\bar{u}(x)] d x \simeq 0.2$ \\
Chiral-quark soliton & 0.31 & {$[129]$} \\
Chiral-quark soliton & $\simeq \int_{0}^{1} 2 x^{0.12}[\bar{d}(x)-\bar{u}(x)] d x$ & {$[131]$} \\
Instanton & $=\frac{5}{3} \int_{0}^{1}[\bar{d}(x)-\bar{u}(x)] d x \simeq 0.2$ & {$[123]$} \\
Statistical & $\simeq \int_{0}^{1}[\bar{d}(x)-\bar{u}(x)] d x \simeq 0.12$ & {$[41]$} \\
Statistical & $>\int_{0}^{1}[\bar{d}(x)-\bar{u}(x)] d x>0.12$ & {$[126]$} \\
\hline
\end{tabular}

Equation 39, together with the constraints of the valence quark sum rules and inputs from polarized DIS experiments, can readily lead to the prediction that $\bar{d}>\bar{u}$ and $\Delta \bar{u}>0>\Delta \bar{d}$.

Predictions of various model calculations for $I_{\Delta}$, the first moment of $\Delta \bar{u}(x)-\Delta \bar{d}(x)$, are listed in Table 5. While the meson cloud model gives small negative values for $I_{\Delta}$, all other models predict a positive $I_{\Delta}$ with a magnitude comparable or greater than the corresponding integral for unpolarized sea (recall that $\int_{0}^{1}[\bar{d}(x)-\bar{u}(x)] d x \simeq 0.12$ ). Several calculations for the direct contribution of $\rho$ meson cloud are in good agreement. However, the large $\pi-\rho$ interference effect reported in [118] was not confirmed in a later study [119]. It is worth noting that Ref. [132] considers $\pi-\sigma$ interference and predicts a large effect on $\Delta \bar{d}-\Delta \bar{u}$, with a sign opposite to other meson cloud model calculations.

If the flavor asymmetry of the polarized sea is indeed as large as the predictions of many models shown in Table 5, it would imply that a significant fraction of the Bjorken sum, $\int_{0}^{1}\left[g_{1}^{p}(x)-g_{1}^{n}(x)\right] d x$, comes from the flavor asymmetry of polarized nucleon sea.

\subsubsection{Experimental status on the asymmetry between $\Delta \bar{u}(x)$ and $\Delta \bar{d}(x)$}

The experimental information on the helicity distributions of the partons is mostly from polarized DIS experiments involving longitudinally polarized lepton beams and polarized targets. From the measured longitudinal $\left(A_{\|}\right)$and transverse $\left(A_{\perp}\right)$ asymmetries

$$
A_{\|}=\frac{d \sigma^{\rightarrow \rightarrow}-d \sigma^{\rightarrow \Leftarrow}}{d \sigma^{\rightarrow \rightarrow}+d \sigma^{\rightarrow \Leftarrow}} ; A_{\perp}=\frac{d \sigma^{\rightarrow \Uparrow}-d \sigma^{\rightarrow \Uparrow}}{d \sigma^{\rightarrow \Uparrow}+d \sigma^{\rightarrow \Uparrow}}
$$

the polarized structure function $g_{1}\left(x, Q^{2}\right)$ can be extracted. In the parton model, $g_{1}\left(x, Q^{2}\right)$ at LO is given as

$$
g_{1}\left(x, Q^{2}\right)=\frac{1}{2} \sum_{i=1}^{n_{f}} e_{i}^{2}\left(\Delta q_{i}\left(x, Q^{2}\right)+\Delta \bar{q}_{i}\left(x, Q^{2}\right)\right),
$$

where $\Delta q_{i}\left(x, Q^{2}\right)=q_{i}^{\uparrow}\left(x, Q^{2}\right)-q_{i}^{\downarrow}\left(x, Q^{2}\right)$ and $\Delta \bar{q}_{i}\left(x, Q^{2}\right)=\bar{q}_{i}^{\uparrow}\left(x, Q^{2}\right)-\bar{q}_{i}^{\downarrow}\left(x, Q^{2}\right)$. At NLO, the $Q^{2}$ dependence of $g_{1}\left(x, Q^{2}\right)$ contains a contribution from $\Delta g\left(x, Q^{2}\right)=g^{\uparrow}\left(x, Q^{2}\right)-g^{\downarrow}\left(x, Q^{2}\right)$ through the term $\frac{\alpha_{s}}{2 \pi} \Delta C_{g} \otimes g$, and can be used to extract gluon polarization. 
Table 6: Polarized SIDIS experiments and results on the first moments of $\Delta \bar{u}(x), \Delta \bar{d}(x), \Delta \bar{u}(x)-\Delta \bar{d}(x)$ and $\Delta \bar{u}(x)+\Delta \bar{d}(x)$. The values of $\left\langle Q^{2}\right\rangle$ for the SMC, HERMES, and COMPASS experiments are 10 $\mathrm{GeV}^{2}, 2.5 \mathrm{GeV}^{2}$, and $3.0 \mathrm{GeV}^{2}$, respectively.

\begin{tabular}{|c|c|c|c|c|}
\hline Experiment & beam/target & det. part. & $\begin{array}{c}\Delta \bar{u} \\
(\Delta \bar{d})\end{array}$ & $\begin{array}{c}\Delta \bar{u}-\Delta \bar{d} \\
(\Delta \bar{u}+\Delta \bar{d})\end{array}$ \\
\hline EMC [133] & $\mu$ on $\mathrm{p}$ & $h^{ \pm}$ & - & - \\
\hline SMC [160] & $\mu^{+}$on $\mathrm{p}, \mathrm{d}$ & $h^{ \pm}$ & $\begin{array}{c}0.01 \pm 0.05 \pm 0.02 \\
(0.01 \pm 0.14 \pm 0.12)\end{array}$ & - \\
\hline HERMES 161 & $e^{+}$on $\mathrm{p},{ }^{3} \mathrm{He}$ & $h^{ \pm}$ & $\begin{array}{c}-0.01 \pm 0.02 \pm 0.03 \\
(-0.02 \pm 0.03 \pm 0.04)\end{array}$ & - \\
\hline HERMES [162] & $e^{+}$on $\mathrm{d}$ & $\pi^{ \pm}, K^{ \pm}$ & $\sim 0$ & $\sim 0$ \\
\hline HERMES [163] & $e^{+}$on $\mathrm{p}, \mathrm{d}$ & $\pi^{ \pm}, K^{ \pm}$ & $\begin{array}{l}-0.002 \pm 0.0036 \pm 0.023 \\
(-0.054 \pm 0.033 \pm 0.011)\end{array}$ & $0.048 \pm 0.057 \pm 0.028$ \\
\hline COMPASS 164. & $\mu$ on $\mathrm{d}$ & $h^{ \pm}$ & - & $(0.0 \pm 0.04 \pm 0.03)$ \\
\hline COMPASS [165] & $\mu$ on $\mathrm{d}$ & $\pi^{ \pm}, K^{ \pm}$ & - & $(-0.04 \pm 0.03 \pm 0.01)$ \\
\hline COMPASS [166] & $\mu$ on $\mathrm{p}, \mathrm{d}$ & $\pi^{ \pm}, K^{ \pm}$ & $\begin{array}{c}0.02 \pm 0.02 \pm 0.01 \\
(-0.05 \pm 0.03 \pm 0.02)\end{array}$ & $\begin{array}{c}0.06 \pm 0.04 \pm 0.02 \\
(-0.04 \pm 0.03 \pm 0.01)\end{array}$ \\
\hline
\end{tabular}

Extensive measurements of polarized DIS have been carried out at EMC [133], SMC [134], SLAC [135, 136, 137, 138, 139], COMPASS [140, 141], HERMES [142, 143], and JLAB [144, 145]. These data have led to the determination of $\Delta u(x)+\Delta \bar{u}(x), \Delta d(x)+\Delta \bar{d}(x), \Delta s(x)+\Delta \bar{s}(x)$, and $\Delta g(x)$ in several global QCD analyses in the next-to-leading (NLO) framework [146, 147, 148, 149, 150, 151, 152, 153, 154]. These neutral current polarized DIS data on proton, deuteron, and ${ }^{3} \mathrm{He}$ allow the isospin separation of the polarized parton distributions (i.e., separating $\Delta u+\Delta \bar{u}$ from $\Delta d+\Delta \bar{d}$ ). However, these data cannot disentangle the antiquark from the quark helicity distributions. In order to extract the sea-quark polarizations, several other experimental approaches have been considered. They include the polarized semi-inclusive DIS, polarized Drell-Yan [155, single-spin asymmetry in $W$-boson production in $p p$ collision [156], and charged-current DIS using neutrino beam at neutrino factory [157] or electron beam at electron ion collider [158]. Among these various approaches, both the polarized SIDIS and the polarized $W$-boson production have been actively pursued in the last decades. In the following, we discuss their impact on the determination of $\Delta \bar{u}(x)$ and $\Delta \bar{d}(x)$.

The first polarized semi-inclusive DIS measurement was reported by the EMC collaboration [133], followed by the SMC [159, 160] and the more recent extensive work at HERMES [161, 162, 163] and COMPASS [164, 165, 166]. As shown in Table 6, these polarized SIDIS experiments use longitudinally polarized $e^{ \pm}, \mu^{ \pm}$beams on a variety of longitudinally polarized $p, d,{ }^{3} \mathrm{He}$ targets. Either unidentified charged hadrons $\left(h^{ \pm}\right)$or identified $\pi^{ \pm}, K^{ \pm}$are detected. From the measured asymmetry $A_{\text {meas }}^{h}$, which is the asymmetry of the normalized count rates when the beam and target polarization are anti-aligned or aligned, the photo-absorption cross section asymmetry, $A_{1}^{h}$, is determined as

$$
A_{1}^{h}=\frac{\sigma_{1 / 2}^{h}-\sigma_{3 / 2}^{h}}{\sigma_{1 / 2}^{h}+\sigma_{3 / 2}^{h}},
$$

where $\sigma_{1 / 2(3 / 2)}^{h}$ refers to the semi-inclusive cross section for producing hadron of type $h$ for photons with spin anti-parallel (parallel) to the target nucleon spin. Since the spin-1 photon can only be absorbed when the quark spin is in the direction opposite to the photon's spin direction, the asymmetry between $\sigma_{1 / 2}^{h}$ and $\sigma_{3 / 2}^{h}$ is sensitive to the helicity distribution of the struck quark (or antiquark). The charge and 
type of the detected hadron will further allow the flavor separation of the quark helicity distribution. $A_{1}^{h}$ can be written as follows:

$$
A_{1}^{h}\left(x, Q^{2}, z\right)=\frac{\sum_{q} e_{q}^{2} \Delta q\left(x, Q^{2}\right) D_{q}^{h}\left(z, Q^{2}\right)}{\sum_{q} e_{q}^{2} q\left(x, Q^{2}\right) D_{q}^{h}\left(z, Q^{2}\right)},
$$

where $D_{q}^{h}\left(z, Q^{2}\right)$ is the fragmentation function of a struck quark with flavor $q$ hadronizing into a hadron $h$ carrying a fraction $z$ of the virtual photon's energy.

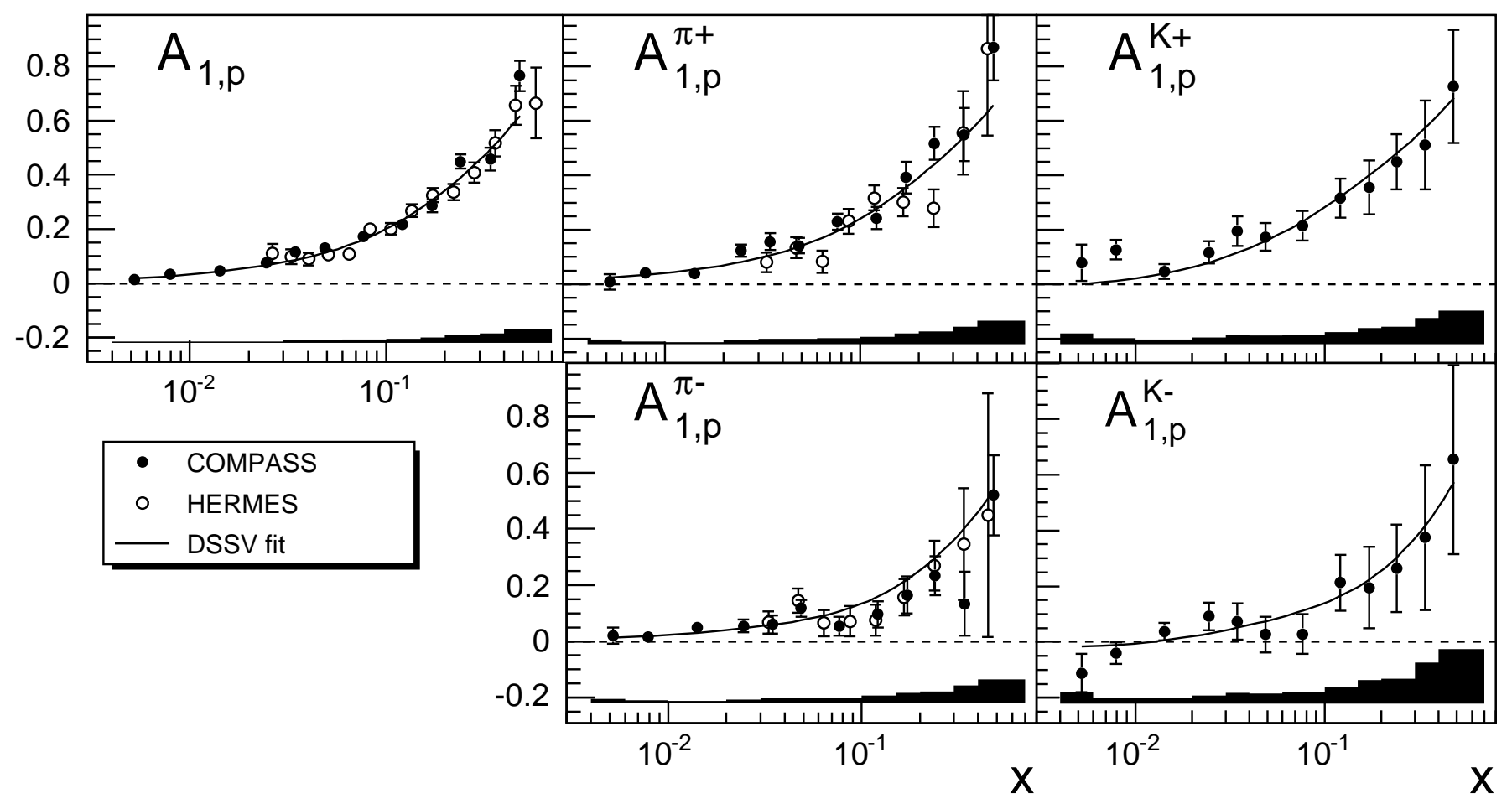

Figure 25: The inclusive asymmetry $A_{1, p}$ and semi-inclusive asymmetries $A_{1, p}^{h}$ from HERMES [143] and COMPASS [166]. The curves are predictions of the DSSV parametrization [167].

Figure 25] shows the $x$ dependence of the inclusive DIS asymmetry $A_{1, p}$ and the semi-inclusive DIS asymmetries $A_{1, p}^{\pi^{ \pm}, K^{ \pm}}$measured on polarized proton targets from HERMES and COMPASS. Very similar trends, showing significant asymmetries at the large- $x$ valence quark region and a gradual fall-off as $x$ approaches the small- $x$ sea-quark region, are observed for all asymmetries. This clearly indicates that the quark polarization is predominantly a valence-quark effect with a relatively small role played by the sea quarks. Nevertheless, the HERMES and COMPASS collaborations have reported their extractions of $\Delta \bar{u}(x)$ and $\Delta \bar{d}(x)$ using polarized semi-inclusive DIS data [163, 166]. Although the early polarized SIDIS measurements were all consistent with negligible $\bar{u}$ and $\bar{d}$ polarizations, the recent analysis from COMPASS suggests a negative first moment for $\bar{d}(x)$ and an intriguing $\Delta \bar{u}(x)-\Delta \bar{d}(x)$ distribution shown in Fig. 26. Although the accuracy of the measurement is still quite limited, the data favor the models which predict positive values for $\Delta \bar{u}(x)-\Delta \bar{d}(x)$, such as the statistical model and the chiral-quark soliton model. 


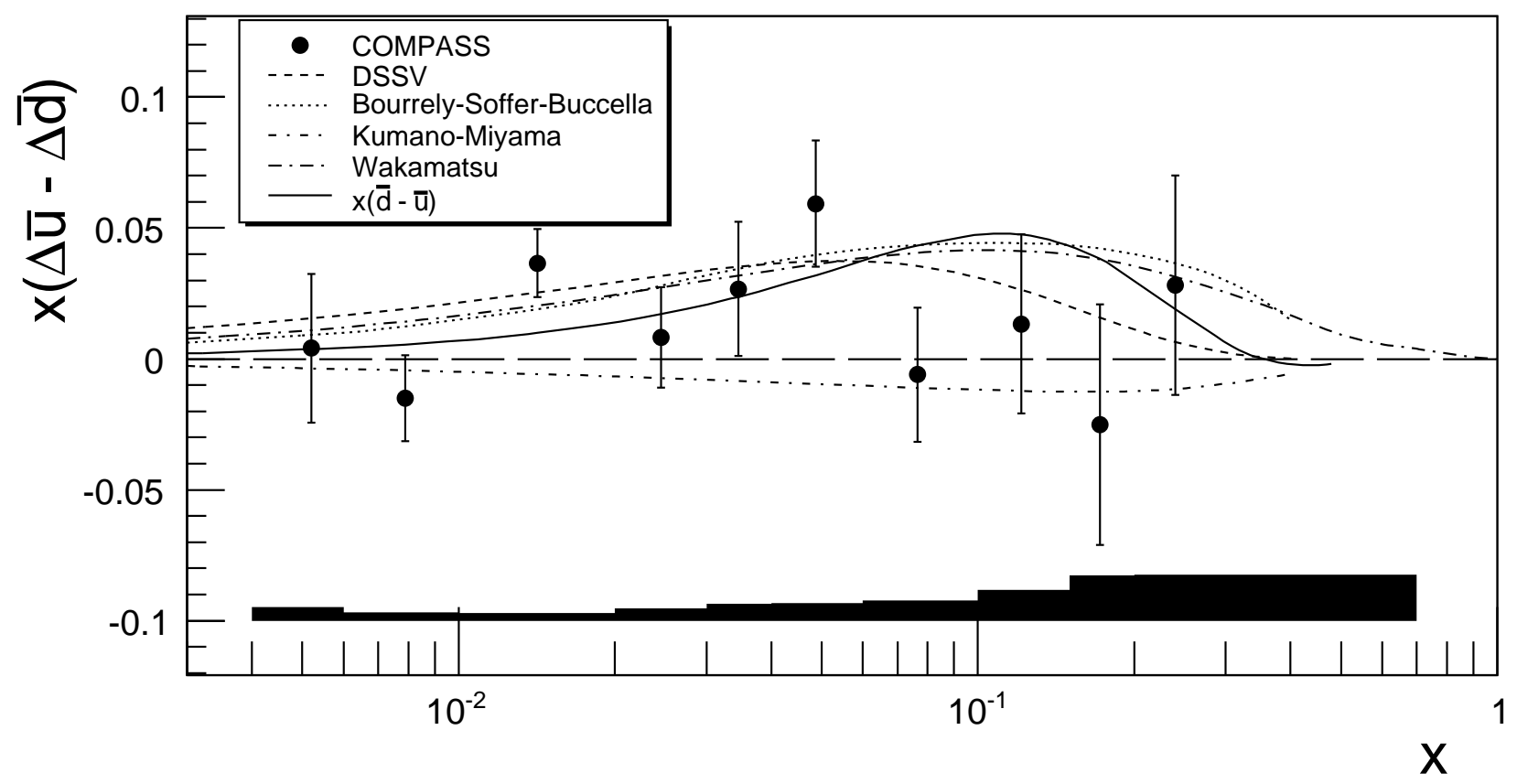

Figure 26: Comparison of $x(\Delta \bar{u}-\Delta \bar{d})$ extracted from COMPASS [166] with the DSSV parametrization and various model predictions.

Figure 26 also shows the comparison between the extracted values of $x(\Delta \bar{u}(x)-\Delta \bar{d}(x))$ and the DSSV parametrization of polarized PDF [167]. Unlike the earlier global fits to the polarized DIS data, the DSSV global fit also included the polarized semi-inclusive DIS data as well as hadron production data from polarized $p p$ collision at RHIC. The DSSV global fit also adopted the latest fragmentation functions [168] which provide a good description of the unpolarized SIDIS data. Since the earlier HERMES and COMPASS polarized SIDIS data were included in the DSSV global fit, it is to be expected that the latest $\Delta \bar{u}(x)-\Delta \bar{d}(x)$ result from COMPASS is well described by DSSV. Nevertheless, the uncertainties in the fragmentation function are a main source of the systematic uncertainties in the extraction of sea-quark polarization, as emphasized in Ref. [166, 169].

Figure 27 shows the polarized sea and gluon distributions from the DSSV global fit. The assumption of an $\mathrm{SU}(3)$ symmetric polarized sea, $\Delta \bar{u}(x)=\Delta \bar{d}(x)=\Delta \bar{s}(x)$, is clearly at a variance with the DSSV best-fit results. However, the error bands for $\Delta \bar{u}(x)$ and $\Delta \bar{d}(x)$ in Fig. 27 are still quite broad. In particular, the narrower $\Delta \chi^{2}=1$ error band gives the first moments of $\Delta \bar{u}=0.028 \pm 0.021$ and $\Delta \bar{d}=-0.089 \pm 0.029$. For the more conservative error band $\left(\Delta \chi^{2} / \chi^{2}=2 \%\right)$, both $\Delta \bar{u}$ and $\Delta \bar{d}$ are consistent with zero $(\Delta \bar{u}=0.028 \pm 0.059$ and $\Delta \bar{d}=-0.089 \pm 0.090)$.

A unique experimental tool for measuring $\Delta \bar{u}$ and $\Delta \bar{d}$ sea-quark polarization is $W$ production in polarized $p p$ collision at RHIC [156, 170]. As discussed by Bourrely and Soffer [156], three parityviolating asymmetries in $W$ production are defined as

$$
A_{L}=\frac{\sigma_{+}-\sigma_{-}}{\sigma_{+}+\sigma_{-}}, \quad A_{L L}^{P V}=\frac{\sigma_{++}-\sigma_{--}}{\sigma_{++}+\sigma_{--}}, \quad \bar{A}_{L L}^{P V}=\frac{\sigma_{+-}-\sigma_{-+}}{\sigma_{+-}+\sigma_{-+}}
$$

where,+- in $A_{L L}$ refers to the proton beam helicity, while $\sigma_{+}=\left(\sigma_{++}+\sigma_{+-}\right) / 2$ and $\sigma_{-}=\left(\sigma_{-+}+\sigma_{--}\right) / 2$ in $A_{L}$. Ignoring the contributions from strange and heavier quarks, the $W^{+}$differential cross section can be written as

$$
\frac{d \sigma}{d y}\left(W^{+}\right)=K \frac{\sqrt{2} \pi}{3} G_{F} x_{1} x_{2} \cos ^{2} \theta_{c}\left[u\left(x_{1}\right) \bar{d}\left(x_{2}\right)+\bar{d}\left(x_{1}\right) u\left(x_{2}\right)\right] .
$$




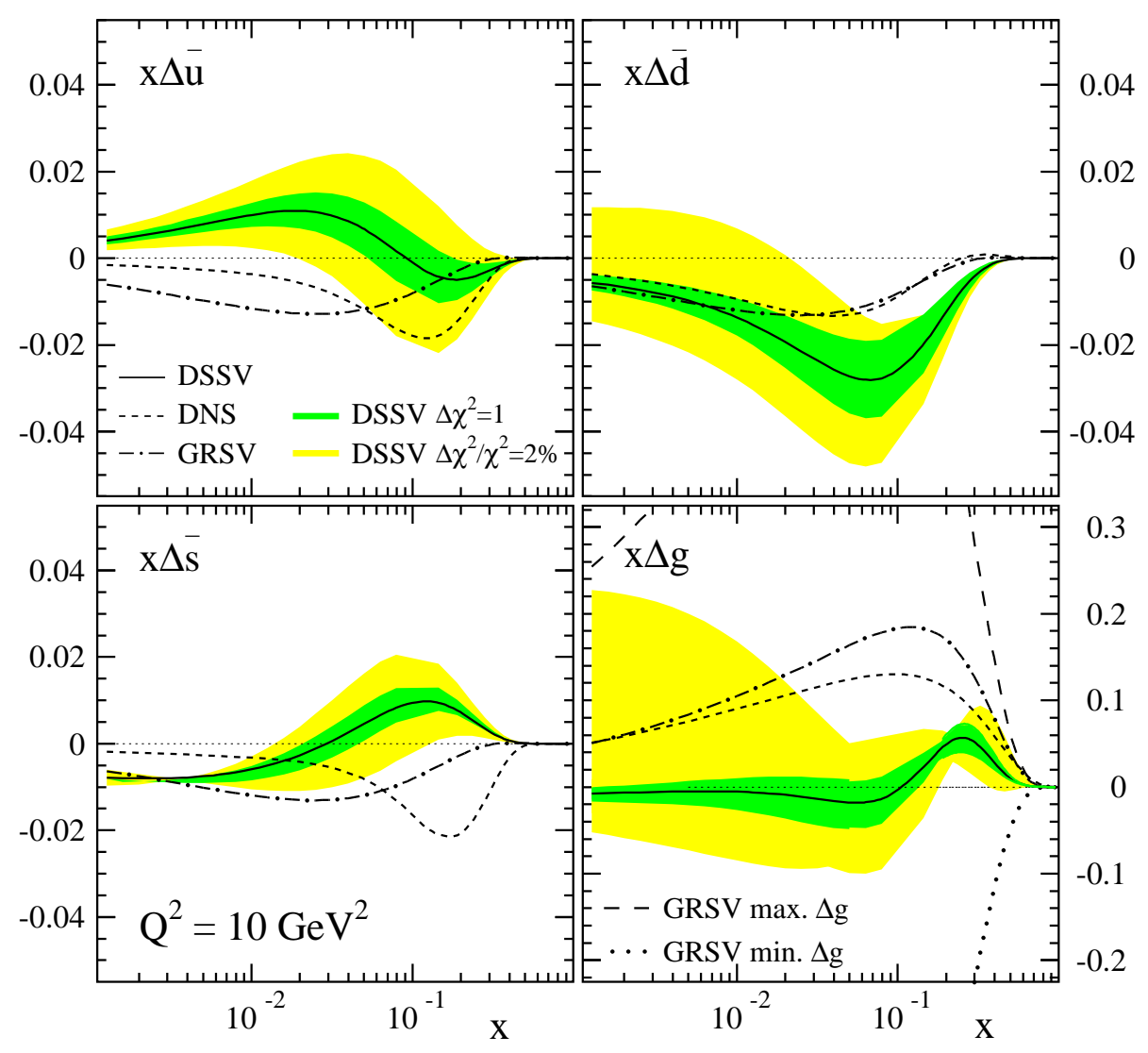

Figure 27: The DSSV polarized sea and gluon distributions [167]. The shaded bands correspond to $\Delta \chi^{2}=1$ and $\Delta \chi^{2} / \chi^{2}=2 \%$. Other polarized PDFs are also shown for comparison.

The factor $K$ takes into account the first-order QCD corrections, $K \simeq 1+\frac{8 \pi}{9} \alpha_{s}\left(Q^{2}\right) \sim 1.323$ at the $W$ mass scale. Since $W$ couples to left-handed quarks and right-handed antiquarks, the expression for the four $W^{+}$differential cross sections are

$$
\begin{array}{rlrl}
\sigma_{++} & \sim u^{\downarrow}\left(x_{1}\right) \bar{d}^{\uparrow}\left(x_{2}\right)+\bar{d}^{\uparrow}\left(x_{1}\right) u^{\downarrow}\left(x_{2}\right) ; & \sigma_{+-} \sim u^{\downarrow}\left(x_{1}\right) \bar{d}^{\downarrow}\left(x_{2}\right)+\bar{d}^{\uparrow}\left(x_{1}\right) u^{\uparrow}\left(x_{2}\right) ; \\
\sigma_{-+} \sim u^{\uparrow}\left(x_{1}\right) \bar{d}^{\uparrow}\left(x_{2}\right)+\bar{d}^{\downarrow}\left(x_{1}\right) u^{\downarrow}\left(x_{2}\right) ; & \sigma_{--} \sim u^{\uparrow}\left(x_{1}\right) \bar{d}^{\downarrow}\left(x_{2}\right)+\bar{d}^{\downarrow}\left(x_{1}\right) u^{\uparrow}\left(x_{2}\right) .
\end{array}
$$

The superscripts $\uparrow, \downarrow$ refer to parton's helicity parallel or anti-parallel to the proton's helicity. Eq. 46 shows that the four beam-helicity dependent $W^{+}$cross sections could lead to the measurement of $u^{\uparrow}(x), u^{\downarrow}(x), \bar{d}^{\uparrow}(x)$, and $\bar{d}^{\downarrow}(x)$. Similarly, the four helicity dependent $W^{-}$cross sections can determine $d^{\uparrow}(x), d^{\downarrow}(x), \bar{u}^{\uparrow}(x)$, and $\bar{u}^{\downarrow}(x)$.

The three parity-violating asymmetries as a function of $W^{+}$'s rapidity $y$ become [156]

$$
\begin{aligned}
A_{L}(y) & =\frac{\Delta \bar{d}\left(x_{1}\right) u\left(x_{2}\right)-\Delta u\left(x_{1}\right) \bar{d}\left(x_{2}\right)}{\bar{d}\left(x_{1}\right) u\left(x_{2}\right)+u\left(x_{1}\right) \bar{d}\left(x_{2}\right)} \\
A_{L L}^{P V}(y) & =\frac{\left[u\left(x_{1}\right) \Delta \bar{d}\left(x_{2}\right)-\Delta u\left(x_{1}\right) \bar{d}\left(x_{2}\right)\right]-\left[\bar{d}\left(x_{1}\right) \Delta u\left(x_{2}\right)-\Delta \bar{d}\left(x_{1}\right) u\left(x_{2}\right)\right]}{\left[\Delta u\left(x_{1}\right) \Delta \bar{d}\left(x_{2}\right)-u\left(x_{1}\right) \bar{d}\left(x_{2}\right)\right]+\left[\Delta \bar{d}\left(x_{1}\right) \Delta u\left(x_{2}\right)-\bar{d}\left(x_{1}\right) u\left(x_{2}\right)\right]} \\
\bar{A}_{L L}^{P V}(y) & =\frac{\left[\bar{d}\left(x_{1}\right) \Delta u\left(x_{2}\right)+\Delta \bar{d}\left(x_{1}\right) u\left(x_{2}\right)\right]-\left[u\left(x_{1}\right) \Delta \bar{d}\left(x_{2}\right)+\Delta u\left(x_{1}\right) \bar{d}\left(x_{2}\right)\right]}{\left[\bar{d}\left(x_{1}\right) u\left(x_{2}\right)+\Delta \bar{d}\left(x_{1}\right) \Delta u\left(x_{2}\right)\right]+\left[u\left(x_{1}\right) \Delta \bar{d}\left(x_{2}\right)+\Delta u\left(x_{1}\right) \Delta \bar{d}\left(x_{2}\right)\right]} .
\end{aligned}
$$



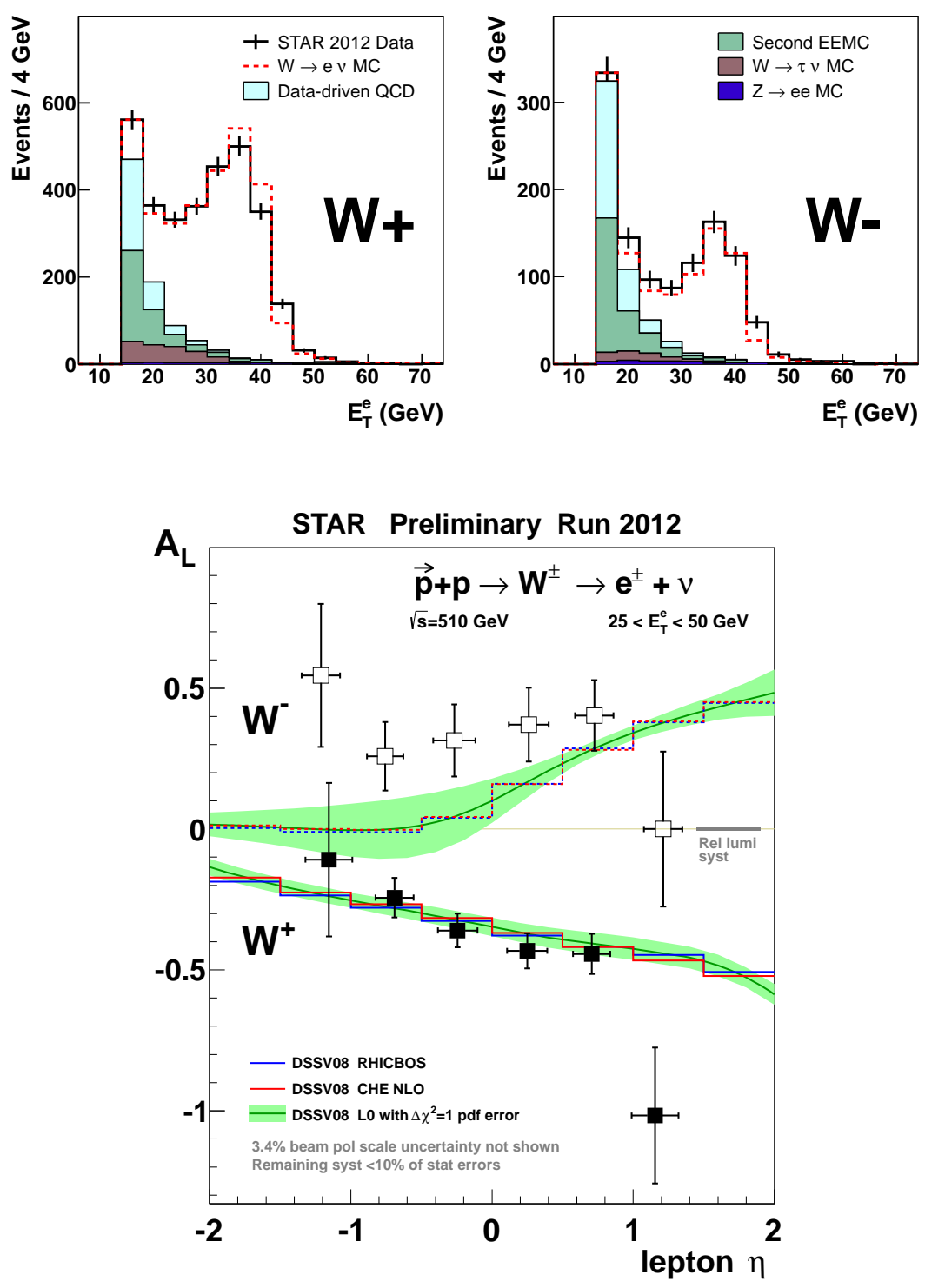

Figure 28: Upper: (a): $W^{+}$and $W^{-}$candidate events from STAR. Lower: (b): Single spin asymmetry $A_{L}$ from STAR [175].

From Eq. 47, it is clear that $A_{L}, A_{L L}^{P V}, \bar{A}_{L L}^{P V}$, together with the unpolarized cross section $\sigma(y)$, would allow the extraction of $u(x), \Delta u(x), \bar{d}(x)$, and $\Delta \bar{d}(x)$ from $W^{+}$production. Similarly, the three parityviolating observables and $\sigma(y)$ for $W^{-}$production would determine $d(x), \Delta d(x), \bar{u}(x)$, and $\Delta \bar{u}(x)$, in principle. In practice, however, it is the rapidity of the charged lepton $\left(l^{ \pm}\right)$in the $W^{ \pm} \rightarrow l^{ \pm} \nu(\bar{\nu})$ decay, rather than $W^{ \pm}$itself, which is measured experimentally. Moreover, as pointed out by Bourrely and Soffer [156], under the reasonable assumption that $\Delta u \Delta \bar{d} \ll u \bar{d}$ (and $\Delta d \Delta \bar{u} \ll d \bar{u}$ ), both $A_{L L}^{P V}(y)$ and $\bar{A}_{L L}^{P V}(y)$ are related to the single spin asymmetry $A_{L}$ :

$$
A_{L L}^{P V}(y)=A_{L}(y)+A_{L}(-y) ; \quad \bar{A}_{L L}^{P V}(y)=A_{L}(y)-A_{L}(-y)
$$

Effectively, there is only a single independent asymmetry observable, $A_{L}(y)$, in polarized $W$ production. From Eq. 47, it is clear that at large forward rapidity region $\left(x_{1} \gg x_{2}\right)$,

$$
A_{L}^{W^{+}} \sim-\frac{\Delta u\left(x_{1}\right)}{u\left(x_{1}\right)}, \quad A_{L}^{W^{-}} \sim-\frac{\Delta d\left(x_{1}\right)}{d\left(x_{1}\right)}
$$


while at large negative rapidity region $\left(x_{1} \ll x_{2}\right)$,

$$
A_{L}^{W^{+}} \sim \frac{\Delta \bar{d}\left(x_{1}\right)}{\bar{d}\left(x_{1}\right)}, \quad A_{L}^{W^{-}} \sim \frac{\Delta \bar{u}\left(x_{1}\right)}{\bar{u}\left(x_{1}\right)} .
$$

Eq. 50 shows that the most sensitive region for determining the sea-quark polarization, $\Delta \bar{u}$ and $\Delta \bar{d}$, is at large negative rapidity region. An important advantage of $W^{ \pm}$production for extracting $\Delta \bar{u}$ and $\Delta \bar{d}$ is that the uncertainty of fragmentation functions encountered in polarized SIDIS is totally absent. The $W$ production mechanism is well understood and theoretical calculations based on NLO (CHE) [171] and resummation (RHICBOS) [172] are available.

First measurement of $A_{L}$ of $W^{ \pm}$production at RHIC has been reported by PHENIX [173] and STAR [174] using data collected in 2009 at $\sqrt{s}=500 \mathrm{GeV}$ with $\sim 10 \mathrm{pb}^{-1}$ integrated luminosity and an average beam polarization of $30 \%$. A significantly higher integrated luminosity of $72 \mathrm{pb}^{-1}$ at $\sqrt{s}=510 \mathrm{GeV}$ and a beam polarization of $56 \%$ during the 2012 run allowed an improved measurement of $A_{L}$. Preliminary results from STAR [175] on the $W \rightarrow e \nu$ candidate events are shown in Fig. 28. The measured $A_{L}$ over the pseudo rapidity region $|\eta|<1.3$ are compared with theoretical predictions from calculations using the DSSV proton helicity distributions. As discussed above, the $A_{L}^{W^{+}}\left(A_{L}^{W^{-}}\right)$ at negative rapidity is sensitive to $\Delta \bar{d} / \bar{d}(\Delta \bar{u} / \bar{u})$. The good agreement between data and prediction for $A_{L}^{W^{+}}$supports the DSSV parametrization for $\Delta \bar{d}(x)$. Interestingly, the preliminary STAR result for

$A_{L}^{W^{-}}$suggests a significantly more positive $\Delta \bar{u}(x)$ than the DSSV parametrization. These new STAR results have prompted a new DSSV ++ global analysis reported in Ref. [176]. A significant shift from negative to positive values of the $\Delta \bar{u}$ moment over $0.05<x<1$ is reported. This clearly illustrates the unique and powerful constraints on $\Delta \bar{u}(x)$ ad $\Delta \bar{d}(x)$ provided by the polarized $W$ production data.

Very recently, RHIC completed a successful run in 2013, and the combined $2012+2013$ integrated luminosity reaches $\sim 230 \mathrm{pb}^{-1}$ [177]. Furthermore, the STAR forward calorimeter together with the PHENIX forward muon spectrometer [178] extend the rapidity coverage to $-2.2<\eta<2.4$, allowing access to the kinematic region highly sensitive to $\Delta \bar{u}$ and $\Delta \bar{d}$. The significantly increased integrated luminosity would also lead to measurements of the sea-quark polarization through the double-spin asymmetries, $A_{L L}^{P V}$ and $\bar{A}_{L L}^{P V}$, which could provide further consistency checks.

\subsection{Polarization of Strange Quark Sea: $\Delta s(x)$ and $\Delta \bar{s}(x)$}

The helicity structures of the strange quark sea, $\Delta s(x)$ and $\Delta \bar{s}(x)$, have attracted much theoretical and experimental interest during the recent decades. The first indication for a sizable polarization of the strange quark sea came from the measurement of the first moment of the structure function $g_{1}\left(x, Q^{2}\right)$ from the EMC experiment [179]. In the scaling limit,

$$
\Gamma_{1}^{p}\left(Q^{2}\right)=\int_{0}^{1} g_{1}^{p}\left(x, Q^{2}\right) d x=\frac{1}{36}\left(4 a_{0}+3 a_{3}+a_{8}\right)
$$

where the three axial charges, $a_{0}, a_{3}, a_{8}$, are related to the first moments of the quark helicity distributions as follows:

$$
\begin{aligned}
& a_{0}=(\Delta u+\Delta \bar{u})+(\Delta d+\Delta \bar{d})+(\Delta s+\Delta \bar{s}) \equiv \Delta \Sigma \\
& a_{3}=(\Delta u+\Delta \bar{u})-(\Delta d+\Delta \bar{d}) \\
& a_{8}=(\Delta u+\Delta \bar{u})+(\Delta d+\Delta \bar{d})-2(\Delta s+\Delta \bar{s})
\end{aligned}
$$

The surprisingly small value of $\Delta \Sigma=0.12 \pm 0.094$ (stat) \pm 0.138 (syst) at $Q^{2}=10.5 \mathrm{GeV}^{2}$, obtained by the EMC Collaboration [179, 180], shows that only a small fraction of the proton's spin is attributed to the quark's spin. It also implies that $\Delta s+\Delta \bar{s}$ has a large negative value [181]. This is due to the 
fact that $a_{3}$ and $a_{8}$ are related to the two constants $F$ and $D$ which can be determined from the weak decay of spin-1/2 baryon octet:

$$
\begin{aligned}
& a_{3}=F+D=g_{A} / g_{V}=1.269 \pm 0.003 \\
& a_{8}=3 F-D=0.586 \pm 0.031
\end{aligned}
$$

where $F=0.464 \pm 0.008$ and $D=0.866 \pm 0.008$ are obtained from hyperon decay data assuming $\mathrm{SU}(3)$ flavor symmetry. Eq. 52 implies

$$
\Delta s+\Delta \bar{s}=\frac{1}{3}\left(a_{0}-a_{8}\right)
$$

The central values of $\Delta \Sigma=0.12$ from EMC and $a_{8}=0.586$ from hyperon decay give $\Delta s+\Delta \bar{s}=-0.155$, a surprisingly large negative value. As discussed later, a more precise later measurement gives a central value of $a_{0}=0.330$, implying $\Delta s+\Delta \bar{s}=-0.085$. This corresponds to a surprisingly large strange-quark polarization in comparison with the lighter $\bar{u}$ and $\bar{d}$ quarks shown in Table 6 .

The assumption of SU(3) flavor symmetry in hyperon decays, needed to derive the expression for $a_{8}$ in Eq. [53, was examined by Savage and Walden [182] who showed that a violation of SU(3) flavor symmetry of up to $25 \%$ may occur. Similar conclusion was found in a more recent work [183]. Nevertheless, recent lattice QCD calculations [184, 185, 186] of hyperon axial couplings support the assumption of SU(3) flavor symmetry.

The uncertainty in $\Delta S$, defined as $\Delta S=\Delta s+\Delta \bar{s}$, also affects the calculation of spin-dependent cross sections for dark-matter scattering off nuclei [187, 188, 189]. The spin-dependent $(\chi+n) /(\chi+p)$ cross section ratio where $\chi$ is the neutralino in the minimal supersymmetric extension of the Standard Model (MSSM), is particularly sensitive to the values of $\Delta S$. Assuming a $2 \sigma$ variation for $\Delta S=-0.09 \pm 0.03$, i.e., $-0.15<\Delta S<-0.03$, the $(\chi+n) /(\chi+p)$ cross section ratio would vary by a factor of $2-3[189$.

In the following, we first summarize the status of various theoretical predictions on the strange quark polarization. The experimental status as well as future prospects will then be presented.

\subsubsection{Theoretical predictions on strange quark polarizations}

Various nucleon structure models, which have predictions at the confinement scale on the $\bar{u}(x) / \bar{d}(x)$ flavor asymmetry as well as $\Delta \bar{u}(x)-\Delta \bar{d}(x)$, also have specific predictions on $\Delta s(x)$ and $\Delta \bar{s}(x)$. In the simplest meson cloud picture of proton consisting of the $K^{+}+\Lambda$ Fock state, the $\bar{s}$ quark residing in the spin-0 $K^{+}$does not carry any net spin, i.e., $\Delta \bar{s}=0$. However, a net polarization of $\Delta s(x)$ is expected since the spin of $s$ quark in $\Lambda$ is aligned with $\Lambda$ 's spin. The relatively small coupling to the kaon cloud implies a small $\Delta s$. By taking into account the $K^{*}(S=1)$ cloud, Cao and Signal [190] found that $\Delta s$ and $\Delta \bar{s}$ are both non-zero, but small $(\Delta s+\Delta \bar{s}=0.01)$.

In the chiral quark model [191, 192, 193], the $u^{\uparrow} \rightarrow K^{+} s^{\downarrow}$ is the dominant process resulting in $\Delta s<0$ and $\Delta \bar{s}=0$. Using the simplest version of the chiral quark model including the Goldstone boson octet $(\pi, K, \eta)$ [191], the moment of $\Delta s(x)$ is related to the moment of $\bar{u}(x)-\bar{d}(x)$, namely, $\Delta s=3 / 2(\bar{u}-\bar{d})<0$. By including the contribution from $\eta^{\prime}$ and the axial $\mathrm{U}(1)$ breaking correction, a prediction of $\Delta s=-0.10$ was obtained [192]. Allowing $\mathrm{SU}(3)$ breaking, a range of $-0.10<\Delta s<-0.05$ was finally predicted [193.

Table 7: First moment of $\Delta S\left(\Delta S=\int_{0}^{1}[\Delta s(x)+\Delta \bar{s}(x)] d x\right)$ from various lattice QCD calculations.

\begin{tabular}{ccccccc}
\hline Reference & {$[194,200$} & {$[195$} & {$[196]$} & {$[197$} & {$[198]$} & {$[199]$} \\
$\Delta S$ & $-0.12(1)$ & $-0.12(7)$ & $-0.019(11)$ & $-0.020(11)$ & $-0.031(17)$ & $-0.0227(34)$ \\
\hline
\end{tabular}


Several lattice QCD calculations for $\Delta S$ have also been reported. Since strange quarks are not present in the valence component of the nucleon, $\Delta S$ only involves the quark-line disconnected diagrams. Pioneering lattice calculations of disconnected matrix elements show large negative values of $\Delta S=$ $-0.12 \pm 0.01$ [194] or $\Delta S=-0.12 \pm 0.07$ [195] consistent with the values deduced from the EMC polarized DIS experiment. More recent lattice calculations, however, found much smaller values for $\Delta S$, as shown in Table 7. The discrepancy is likely due to the use of quenched action in the early work [194, 195]. A full calculation of all the contributions to the proton spin, including the spin of quarks (antiquarks) and gluons and the orbital angular momenta of quarks (antiquarks), was recently reported [200] using a quenched action. An interesting finding of this recent work is that the orbital angular momentum (OAM) of strange quarks has a positive value $\left(2 L_{s}=0.14 \pm 0.01\right)$, which nearly cancels the spin part of the strange quarks $(\Delta S=-0.12 \pm 0.01)$. The net contribution of the strange quarks to the proton's spin is $0.02 \pm 0.01$, which is close to the values obtained by the recent unquenched calculations for $\Delta S$, as shown in Table 7. Note that the OAM for strange quarks only originates from the disconnected diagram. The interplay between the roles of OAM and spin for the strange quarks is an interesting topic deserving further studies [201].

An inherent uncertainty in the extraction of $\Delta S$ from the inclusive or semi-inclusive polarized DIS is the contribution from the unmeasured small- $x$ region. An alternative method which bypasses this uncertainty is to measure the strange quark's contribution to the axial form factor, $G_{A}^{s}\left(Q^{2}\right)$, from $\nu p$ and $\bar{\nu} p$ elastic scattering cross sections. The value of $\Delta S$ can be deduced by extrapolating $G_{A}^{s}\left(Q^{2}\right)$ to $Q^{2}=0$, namely, $\Delta S=G_{A}^{s}\left(Q^{2}=0\right)$. At low $Q^{2}$, the $\nu p$ elastic cross section is dominated by the axial form factor:

$$
\frac{d \sigma}{d Q^{2}}\left(Q^{2} \rightarrow 0\right) \sim \frac{G_{F}^{2}}{32 \pi} \frac{M_{p}^{2}}{E_{\nu}^{2}}\left[\left(G_{A}^{Z}\right)^{2}+\left(1-4 \sin ^{2} \theta_{W}\right)^{2}\right]
$$

where

$$
G_{A}^{Z}=\frac{1}{2}\left(-G_{A}^{u}+G_{A}^{d}+G_{A}^{s}\right)
$$

The $u$ and $d$ contribution to $G_{A}^{Z}$ is well determined from neutron $\beta$ decay, $G_{A}^{\mu}-G_{A}^{d}=g_{A}=1.269 \pm$ 0.003. Several model calculations for $G_{A}^{s}\left(Q^{2}\right)$ using chiral quark soliton model [202, 203], five-quark model [204, 205], chiral quark model [206], and SU(3) skyrme model [207] also exist. Table 8 lists the prediction of these models on $\Delta S=G_{A}^{s}\left(Q^{2}=0\right)$. It should be cautioned that the value of $\Delta S$ deduced from $G_{A}^{s}\left(Q^{2}=0\right)$ corresponds to $Q^{2}=0$, while $\Delta S$ extracted from polarized semi-inclusive DIS corresponds to $Q^{2}$ of several $\mathrm{GeV}^{2}$. It is not obvious how the value of $\Delta S$ obtained at $Q^{2}=0$ can be compared with that obtained at larger $Q^{2}$ values.

Table 8: Predictions of $\Delta S=G_{A}^{s}\left(Q^{2}=0\right)$ from various models.

\begin{tabular}{ccccc}
\hline Reference & {$[202,[203]$} & {$[204,[205]$} & {$[206]$} & {$[207]$} \\
$\Delta S$ & -0.075 & $-0.05 \pm 0.02$ & $-0.0052 \pm 0.0015$ & -0.03 \\
\hline
\end{tabular}

\subsubsection{Experimental status on strange quark polarizations}

There are several experimental tools for probing the helicity distributions of strange quarks. They include the polarized inclusive DIS, the polarized semi-inclusive DIS, neutrino elastic scattering, hyperon weak decays, and the longitudinal spin transfer $D_{L L}$ of $\Lambda$ and $\bar{\Lambda}$ production in polarized $p p$ collision. We briefly summarize the current status on the extraction of strange quark polarization, as well as the impact of these data on the parametrization of recent polarized PDFs. 
As discussed in Section 4.1.2, extensive measurement of polarized inclusive DIS has led to the determination of $\Delta u(x)+\Delta \bar{u}(x), \Delta d(x)+\Delta \bar{d}(x), \Delta s(x)+\Delta \bar{s}(x)$, and $\Delta g(x)$ in several QCD analyses. Based on their measurements of $g_{1}^{p}\left(x, Q^{2}\right)$ and $g_{1}^{d}\left(x, Q^{2}\right)$, the HERMES Collaboration determined the first moment of the strange quark helicity distribution to be $\Delta s+\Delta \bar{s}=-0.085 \pm 0.018$ at $Q^{2}=5$ $\mathrm{GeV}^{2}$ [143] in the NNLO analysis. From a measurement of $g_{1}^{d}\left(x, Q^{2}\right)$ alone, the COMPASS Collaboration extracted $\Delta s+\Delta \bar{s}=-0.08 \pm 0.01$ (stat) \pm 0.02 (syst) at $Q^{2}=3 \mathrm{GeV}^{2}$ [140]. While these are very important results, the assumption of $\mathrm{SU}(3)$ symmetry was adopted in the analysis. Moreover, as discussed earlier, the inclusive DIS data do not facilitate the separation of $\Delta s$ from $\Delta \bar{s}$.
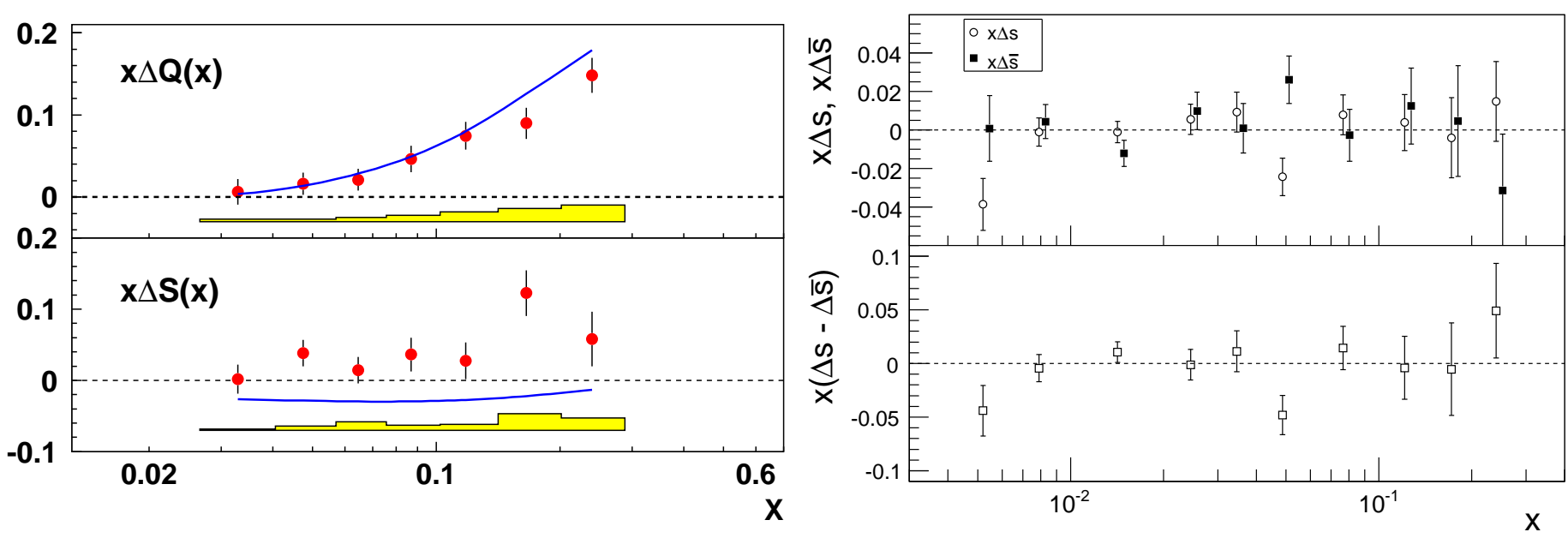

Figure 29: Left (a): Nonstrange and strange quark helicity distributions at $Q^{2}=2.5 \mathrm{GeV}^{2}$ from HERMES [74]. The curves are from the global fit of Leader et al. [208]. Right (b): Extraction of $x \Delta s(x), x \Delta \bar{s}(x)$, and $x(\Delta s(x)-\Delta \bar{s}(x))$ from COMPASS [166].

From polarized semi-inclusive kaon production data, both the HERMES and the COMPASS Collaborations have also reported the extraction of the $x$-dependence of the $\Delta S(x) \equiv \Delta s(x)+\Delta \bar{s}(x)$ distributions. Figure 29 shows the HERMES result on the nonstrange and strange quark helicity distributions at $Q^{2}=2.5 \mathrm{GeV}^{2}[74$. While the nonstrange quark helicity distribution $(\Delta Q(x) \equiv$ $\Delta u(x)+\Delta \bar{u}(x)+\Delta d(x)+\Delta \bar{d}(x))$ is in good agreement with the result obtained by Leader et al. 208] from their global fit to polarized inclusive DIS data, the strange quark helicity distribution $(\Delta S(x))$ favors positive values and differs from the inclusive DIS result. This apparent tension between the extraction of $\Delta S(x)$ using inclusive DIS versus semi-inclusive DIS was also observed by the COMPASS experiment. Figure 29 shows the COMPASS extraction [166] of $\Delta s(x), \Delta \bar{s}(x)$ and $\Delta s(x)-\Delta \bar{s}(x)$. This represents the first flavor separation between $\Delta s(x)$ and $\Delta \bar{s}(x)$, demonstrating a unique capability of polarized semi-inclusive DIS reaction. There is an apparent discrepancy of $\Delta S(x)$ extracted from inclusive DIS versus semi-inclusive DIS. This discrepancy could reflect the uncertainty of the kaon fragmentation functions used in the SIDIS analysis which is absent in the inclusive DIS analysis [166, 209]. Indeed, it was shown [209] that a different choice of the kaon fragmentation function [210] would greatly reduce this discrepancy. Another possible source of this discrepancy could be the assumption of SU(3) symmetry in the inclusive DIS analysis. Several attempts to fit both the inclusive DIS and the semiinclusive DIS data have been carried out [167, 209, 211]. One example of such a combined fit [167] is shown in Fig. 27, in which the $\Delta S(x)$ distribution contains a node at small $x$. This allows positive values of $\Delta S(x)$ at the $x$ region probed by semi-inclusive DIS, while agreeing with the negative value of the moment of $\Delta S(x)$ favored by the DIS analysis. A very recent report from HERMES [75] showed that the updated kaon multiplicity analysis [212] does not lead to any significant difference in the extraction of $\Delta S(x)$. Clearly, more precise data on semi-inclusive DIS, as well as accurate determination of the kaon fragmentation function, are needed for an accurate extraction of the strange quark helicity 
distribution.

The neutral-current neutrino elastic scattering reactions, $\nu_{\mu}+p \rightarrow \nu_{\mu}+p$ and $\bar{\nu}_{\mu}+p \rightarrow \bar{\nu}_{\mu}+p$, were measured sometime ago in the E734 experiment [213] at BNL. This experiment led to an extraction of the weak mixing angle $\sin ^{2} \theta_{W}$, as well as the axial-vector form factor $G_{A}\left(Q^{2}\right)$. The E734 analysis suggested an additional contribution to $G_{A}\left(Q^{2}\right)$ possibly originating from the heavy-quark currents. A later analysis by Garvey, Louis, and White [214] showed that the strange axial form factor $G_{1}^{s}\left(Q^{2}\right)$ can be extracted, provided that strange vector form factors are better known. An extensive effort to determine the strange quark vector form factors has been conducted in several parity-violating electron nucleon scattering experiments at Bates, JLab, and Mainz (see Ref. [215] for a recent review). This allows a combined fit [216, 217] to the E734 neutrino scattering data and the parity-violating electron scattering data, leading to the determination of strange vector $\left(G_{E}^{s}\right.$ and $\left.G_{M}^{s}\right)$ and axial $\left(G_{A}^{s}\right)$ form factors. The extracted values of $G_{A}^{s}\left(Q^{2}\right)$ suggest that $\Delta S=G_{A}^{s}\left(Q^{2}=0\right)$ is negative, $\Delta S=-0.30 \pm 0.42$, consistent with the result from polarized DIS experiments. The accuracy of this independent measurement of $\Delta S$ could be significantly improved [218] when the data from MicroBooNE become available. The possibility of utilizing intense neutrino beam from pion decays has also been considered [219].

In polarized $p p$ collision, the longitudinal spin transfer $D_{L L}$ for $\Lambda$ and $\bar{\Lambda}$ production is sensitive to the helicity distributions of $s$ and $\bar{s}$ [220, 221]. A first measurement of $D_{L L}$ at mid-rapidity region for $\Lambda$ and $\bar{\Lambda}$ was reported by the STAR Collaboration [222]. More recently, an eight-fold increase in data sample was collected at $200 \mathrm{GeV}$ [177]. Preliminary result suggests a positive value of $D_{L L}$ for both $\Lambda$ and $\bar{\Lambda}$ at a $p_{T}$ of $6 \mathrm{GeV} / \mathrm{c}$. While the extraction of $\Delta s(x)$ and $\Delta \bar{s}(x)$ is likely to depend on the poorly known fragmentation functions, this process could be an interesting new tool for probing the strange quark helicity distributions.

\section{Transverse Structure of the Nucleon Sea}

In addition to the spin-independent and the helicity distributions of sea quarks discussed so far, there are also other novel sea quark distributions involving transverse degrees of freedom for the nucleon or quarks. These transverse degrees of freedom include the transverse spin of the nucleon and the quark, as well as the transverse momentum of the quark. There has been intense experimental effort in the last decade to measure these novel parton distributions using lepton and hadron beams. These novel distributions invoking the transverse degrees of freedom potentially offer new insights on the nucleon structure. They also provide stringent tests for various models on nucleon structure. Moreover, the progress in lattice QCD also allows comparisons between the lattice results with the experiments.

From the three transverse quantities, namely, the nucleon's transverse spin $\left(\vec{S}_{\perp}^{N}\right)$, the quark's transverse spin $\left(\vec{s}_{\perp}^{q}\right)$, and the quark's transverse momentum $\left(\vec{k}_{\perp}^{q}\right)$, three different correlations could be formed. The correlation between the quark's and the nucleon's transverse spins leads to the "transversity" distribution. The correlation between quark's transverse momentum and the nucleon's transverse spin is the Sivers function. The Boer-Mulders function corresponds to the correlation between the quark's transverse spin and its transverse momentum. Among the various novel parton distributions, the bulk of recent progress centered on these three distributions. In the remainder of this Section we discuss the recent progress related to these three distributions, focusing on their sea-quark components.

\subsection{Transversity Sea}

The nucleon unpolarized and polarized quark distributions, $q\left(x, Q^{2}\right)$ and $\Delta q\left(x, Q^{2}\right)$, are now rather well known. In contrast, a third quark distribution, called transversity $\left(\delta q\left(x, Q^{2}\right)\right.$ or $\left.h_{1}^{q}\left(x, Q^{2}\right)\right)$, was only beginning to be measured during the last decade. The transversity distribution, which can be described in the quark-parton model as the net transverse polarization of quarks in a transversely polarized 
nucleon [223], has many interesting properties:

- In the non-relativistic quark model, where boosts and rotations commute, the transversity distributions are identical to the helicity distributions. The differences between the transversity and the helicity distributions would reflect the relativistic nature of the quarks inside the nucleon.

- The transversity distributions have a valence-like behavior. Since the transversity of gluons in a nucleon does not exist, the transversity distributions are expected to follow a simple evolution as a flavor non-singlet quantity [224].

- The transversity distributions are predicted to obey some inequality relations. The first, $|\delta q(x)| \leq$ $q(x)$, follows from its interpretation as a difference of probabilities. The second (Soffer's bound) has its origins in the positivity of helicity amplitudes [225], $\left|h_{1}^{q}(x)\right| \leq\left(f_{1}^{q}(x)+g_{1}^{q}(x)\right) / 2$. As discussed below, other inequalities can be obtained in the limit of large $N_{c}$.

- The lowest moment of valence-quark transversity distribution measures a simple local operator analogous to the axial charge, known as the "tensor charge". The tensor charge has been calculated in various theoretical models and in lattice QCD.

In the following, we focus our discussion on the sea-quark transversity distribution. We adopt the notation of $\delta q(x)$ and $\delta \bar{q}(x)$ for the quark and antiquark transversity distributions. While various models predict similar behaviors for the valence-quark transversity distributions, the predictions on the sea-quark transversity are sensitive to details of the models.

In the non-relativistic limit, one expects the same helicity and transversity distributions. Therefore, one would expect similar flavor structure for the sea-quark transversity and helicity distributions. However, model calculations for the sea-quark transversity have distinct predictions and do not necessarily follow this expectation. In a chiral-quark soliton model calculation [226] in the large $N_{c}$ limit, it was predicted that $\delta \bar{u}(x)-\delta \bar{d}(x)<0$, which is opposite to the case for helicity, namely, $\Delta \bar{u}(x)-\Delta \bar{d}(x)>0$. A similar result was obtained by Wakamatsu [227], who predicts the first moments of $\delta \bar{u}=-0.05$ and $\delta \bar{d}=0.08$ at $Q^{2}=0.36 \mathrm{GeV}^{2}$. In a statistical model approach [228], $\delta \bar{q}(x)=\kappa \Delta \bar{q}(x)$ is predicted with an estimated value of 0.6 for $\kappa$. This leads to a positive $\delta \bar{u}(x)$ and a negative $\delta \bar{d}(x)$, and hence $\delta \bar{u}(x)-\delta \bar{d}(x)>0$, which is opposite to the predictions of the chiral-quark soliton model [226, 227]. Finally, the very recent attempt to calculate the isovector $\delta \bar{u}(x)-\delta \bar{d}(x)$ in lattice QCD found [229] large negative values in qualitative agreement with the prediction of the chiral quark soliton model. It would be very interesting to measure the sign and magnitude of $\delta \bar{u}(x)$ and $\delta \bar{d}(x)$ to test the conflicting predictions from various models.

Due to the chiral-odd nature of the transversity distribution, it cannot be measured in inclusive DIS experiments. In order to measure $\delta q\left(x, Q^{2}\right)$, an additional chiral-odd object is required. For example, the double spin asymmetry, $A_{T T}$, for Drell-Yan cross section in transversely polarized $p p$ collision, is sensitive to transversity since $A_{T T} \sim \Sigma_{i} e_{i}^{2} \delta q_{i}\left(x_{1}\right) \delta \bar{q}_{i}\left(x_{2}\right)$ [230]. Such a measurement could be carried out at RHIC [170], although the anticipated effect is small, on the order of $1-2 \%$, due to the presumably small size of the sea-quark transversity distributions. However, significantly larger values of $A_{T T}$ are expected if the sea quark transversity is as large as what the lattice QCD predicts [229]. An interesting proposal is to produce polarized antiproton beam at the FAIR facility [231]. This would allow the measurement of $A_{T T}$ for the Drell-Yan process in $\bar{p} p$ collision, involving the valence transversity distributions in $\bar{p}$ and $p$. Much larger values of $A_{T T}$ are expected [231] for $\bar{p} p$ collision than those of $p p$ collision.

Several other methods for measuring transversity have been proposed. In particular, Collins suggested [232] that a novel chiral-odd fragmentation function, called Collins function $\left(H_{1}^{\perp}\right)$, in conjunction with the chiral-odd transversity distribution, would lead to a single-spin azimuthal asymmetry in semiinclusive meson production. Using a transversely polarized target, an azimuthal angular modulation of 


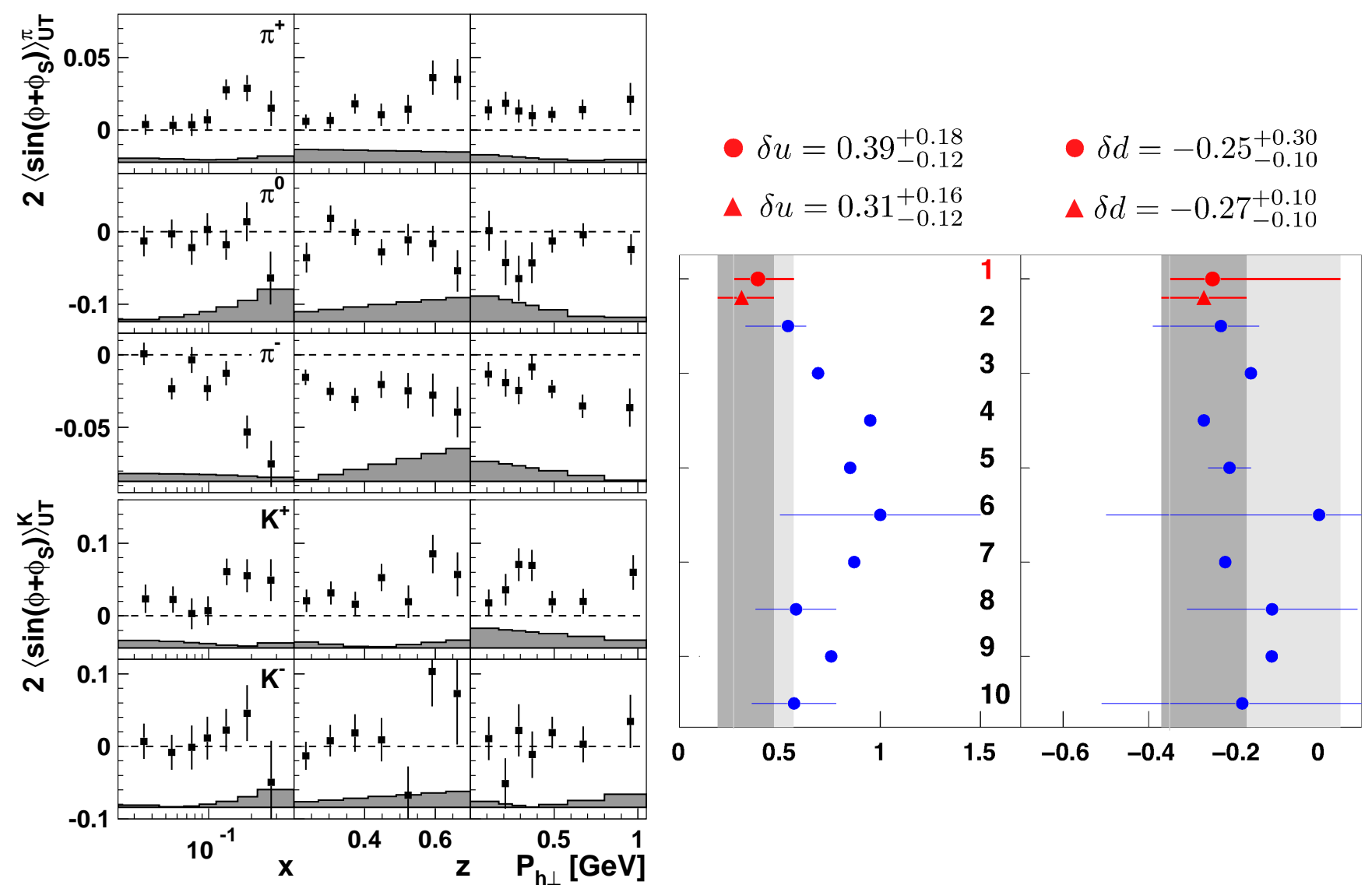

Figure 30: Left (a): Amplitudes for the $\sin \left(\phi_{h}+\phi_{s}\right)$ azimuthal dependence in semi-inclusive DIS measured by the HERMES Collaboration. From [236. Right (b): Tensor charge $\delta u$ and $\delta d$ obtained from the fit to the semi-inclusive DIS data at $Q^{2}=2.41 \mathrm{GeV}^{2}$ in comparison with various calculations [242].

$\sin \left(\phi_{h}+\phi_{s}\right)$ would be proportional to $h_{1} \otimes H_{1}^{\perp}$, which is the convolution of the transversity and the Collins fragmentation function.

The Collins fragmentation function can be extracted from the $\cos 2 \phi$ azimuthal dependence in the $e^{+} e^{-} \rightarrow h^{+} h^{-}$reactions [233]. The observation of a sizable Collins fragmentation function at Belle [234] provided a crucial input for extracting the transversity distribution from the detection of $\sin \left(\phi_{h}+\phi_{s}\right)$ angular modulation in polarized SIDIS.

Major efforts to measure the transversity distributions via SIDIS on transversely polarized targets have been carried out at HERMES [235, 236], COMPASS [237, 238, 239], and JLab [240, 241] during the last decade. An example of the results obtained from these experiments is shown in Fig. 30 (a). The measurement from HERMES [236] using a transversely polarized hydrogen target shows non-zero $\sin \left(\phi+\phi_{s}\right)$ amplitudes for charged pions and $K^{+}$. In contrast, the amplitudes for $K^{-}$are consistent with zero. Since the valence quarks in $K^{-}$have flavors different from the valence quarks in the nucleons, this suggests a small sea quark transversity. Similar results were also reported by the COMPASS Collaboration [239].

Global analysis of the HERMES, COMPASS, and Belle data has led to the extraction of the transversity distributions of the $u$ and $d$ quarks. In the most recent analysis [242] the sea quark transversity was assumed to be zero. The salient feature of the results from this global analysis is that the $u(d)$ quark transversity is positive (negative), just like the $u(d)$ helicity distributions. However, the magnitudes of the transversity distributions are smaller than the corresponding helicity distributions. Figure 30 (b) shows the tensor charges $\delta u$ and $\delta d$, where $\delta q \equiv \int_{0}^{1}[\delta q(x)-\delta \bar{q}(x)] d x$, extracted from this analysis. Predictions from various models and lattice QCD are also shown. It is noted that the central values 
for the tensor charges are $\delta u=0.31$ and $\delta d=-0.27$, which are significantly smaller in magnitude than the corresponding values for the axial charges, $\Delta u=0.787$ and $\Delta d=-0.319$. Figure 30 (b) indicates that the extracted $\delta u$ is significantly smaller than the model predictions. The neglect of the sea quark transversity in the analysis could introduce significant systematic uncertainties in the determination of the tensor charges $\delta u$ and $\delta d$. It is worth noting that the recent result from JLab [241] shows a large negative $\sin \left(\phi+\phi_{s}\right)$ amplitude for $K^{-}$production on a transversely polarized ${ }^{3}$ He target, hinting a sizable sea quark transversity. Future high-statistics measurements proposed at the 12 GeV JLab upgrade [243, as well as measurements of $A_{T T}$ in Drell-Yan process at RHIC are required to pin down the role of sea quarks in the transversity distributions.

\subsection{Sivers Sea}

It was suggested by Sivers [244] that correlations between the transverse spin of the target nucleon and the transverse momentum of the unpolarized quark could lead to single-spin asymmetries in various processes. This correlation is expressed in terms of the "Sivers Function", which is an example of transverse-momentum-dependent (TMD) parton distribution functions. Note that different notations have been adopted for Sivers function, either $f_{1 T}^{\perp}\left(x, k_{\perp}\right)$ or $\Delta^{N}\left(x, k_{\perp}\right)$. As a time-reversal odd object, the Sivers function requires initial/final state interactions via a soft gluon. As shown in [245, 246, 247, such interactions are incorporated in a natural fashion by the gauge link that is required for a gaugeinvariant definition of the TMD parton distribution. An unambiguous measurement of Sivers function would be very valuable for understanding the nature of the TMD parton distributions.

An important feature of the Sivers function is that it is related to the forward scattering amplitude of $N^{\Rightarrow} q \rightarrow N^{\Leftarrow} q$ where the helicity of the target nucleon is flipped. The helicity flip of the nucleon must involve the orbital angular momentum of the unpolarized quark. Therefore, the Sivers function is connected to the angular momentum of the quark. In the meson-cloud model, a fraction of the nucleon's momentum resides in the orbiting mesons. Since the mesons contain valence antiquarks, it is natural to expect that antiquarks carry non-zero orbital angular momentum [248, 249]. Using the chiral quark soliton model, Wakamatsu has shown the striking result that $\bar{u}$ and $\bar{d}$ have dominant contributions to the proton's orbital angular momentum [250]. The recent lattice calculation [200] also found a significant

fraction of proton's spin coming from the $\bar{u}$ and $\bar{d}$ orbital angular momentum. All of these theoretical studies suggest that the Sivers functions for sea quarks could be sizable and measurable.

A first experimental indication for a sizable sea quark Sivers function came from the HERMES measurement of a larger amplitude for the $\sin \left(\phi_{h}-\phi_{s}\right)$ Sivers moment in $K^{+}$relative to $\pi^{+}$production [251]. Other measurements of the Sivers moment have been reported by the HERMES [252] and COMPASS [253, 254] Collaborations. An example is shown in Figure 31 (a) from the HERMES Collaboration [252]. A global analysis to extract both the quark and antiquark Sivers functions was performed 255. Figure 31 (b) shows the results on the extracted Sivers functions for the $u, d$, and various sea quarks. Another global analysis to extract the Sivers function, using more recent data from COMPASS, was recently reported [256]. Nonzero $\bar{u}$ and $\bar{d}$ Sivers functions are also obtained. Although the global analysis suggests non-zero sea-quark Sivers functions, especially for $\bar{d}$, to account for the large Sivers moment observed for $K^{+}$, much better statistical accuracy [243] is required to draw definitive conclusions. 


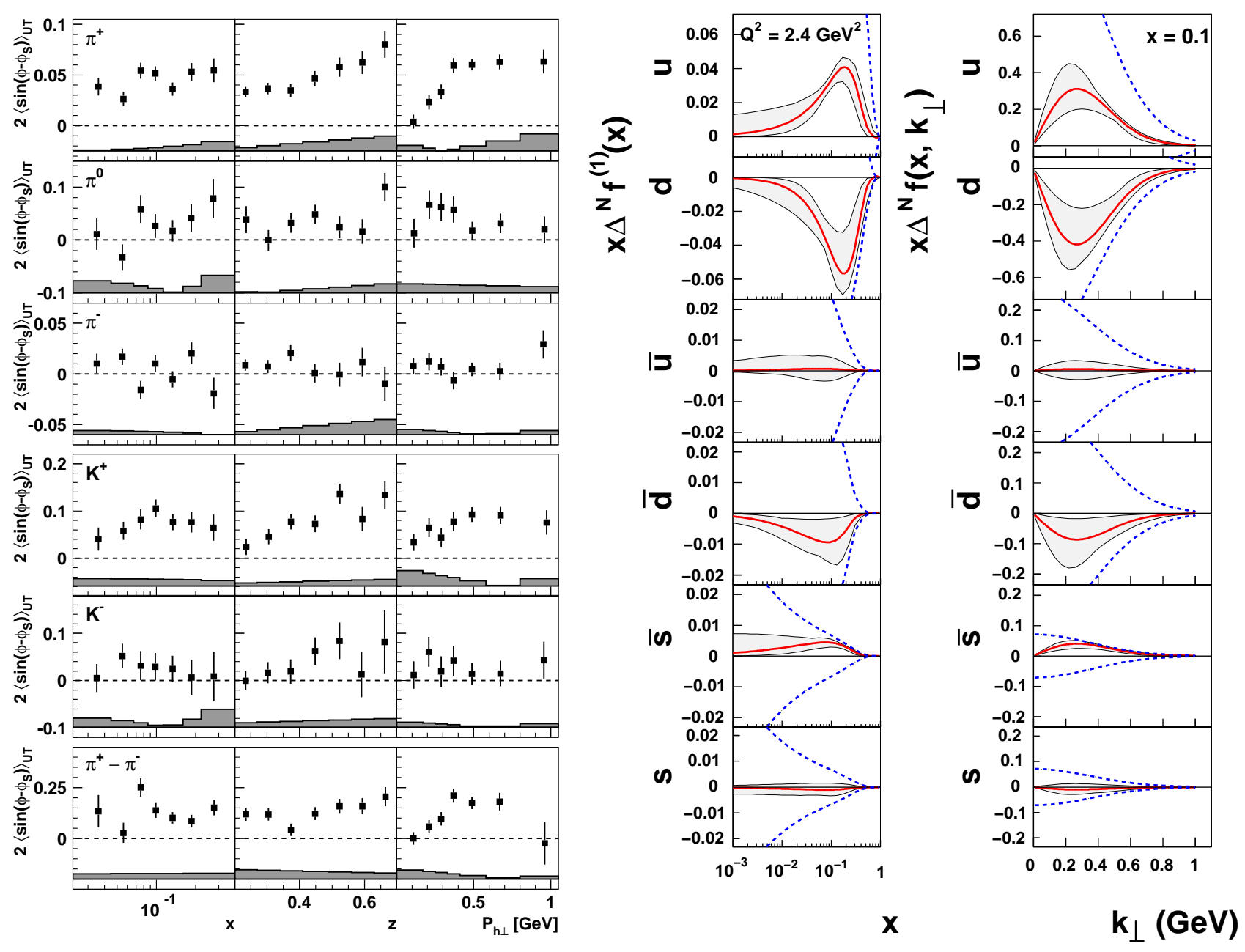

Figure 31: Left (a): Amplitudes for the $\sin \left(\phi_{h}-\phi_{s}\right)$ azimuthal dependence in semi-inclusive DIS measured by the HERMES Collaboration [252. Right (b): Sivers functions for quarks and antiquarks extracted from global analysis of semi-inclusive DIS data [255].

Sivers functions could also be studied in hadron-hadron collisions. The observed large single spin asymmetries in the production of $\bar{p}$ and $K^{-}$from the polarized $p p$ collisions [257] might hint nonvanishing sea-quark Sivers distributions. Polarized $p p$ Drell-Yan experiment provides a clean probe to extract sea quark Sivers distributions without the ambiguities of parton fragmentation. A proposal [258] of replacing the target of the Fermilab E906/SeaQuest DY experiment by a transversely-polarized $\mathrm{NH}_{3}$ target to measure the sea quark Sivers function has been approved.

\subsection{Boer-Mulders Sea}

The Boer-Mulders function [259], $h_{1}^{\perp}\left(x, k_{\perp}\right)$, is another example of a T-odd TMD. It signifies the correlation between $\vec{k}_{\perp}$ and the quark transverse spin, $\vec{s}_{\perp}$, in an unpolarized nucleon. As a chiralodd analog of the Sivers function, the Boer-Mulders function also owes its existence to the presence of initial/final state interactions [245]. Such interactions are incorporated in a natural fashion by the gauge link that is required for a gauge-invariant definition of the TMDs [246].

The flavor and $x$ dependence of the Boer-Mulders functions have been calculated using various models. In the quark-diquark model, the Boer-Mulders functions are shown to be identical to the Sivers functions when the scalar diquark configuration alone is considered [260, 261]. However, calculations taking into account both the axial-vector and the scalar configurations found significant differences in 
the flavor dependence between the Sivers and Boer-Mulders functions [262]. In particular, while the $u$ and $d$ valence quark Boer-Mulders functions are predicted to be both negative, the Sivers function is negative for the $u$ and positive for the $d$ valence quarks. Other model calculations utilizing the MIT bag model [263], the large- $N_{c}$ model [264], the relativistic constituent quark model [265], as well as lattice QCD [266] all predict negative signs for the $u$ and $d$ valence Boer-Mulders functions. It is interesting to test their prediction that the $u$ and $d$ Boer-Mulders functions both have negative signs.

The Sivers functions do not exist for spin-zero hadrons, such as pions, since it corresponds to the correlation between quark's $\vec{k}_{\perp}$ and hadron's spin. On the other hand, the Boer-Mulders functions can exist for pions, since they do not depend on hadron's spin. Calculations for the pion's valence-quark Boer-Mulders functions using the quark-spectator-antiquark model predict [267] a negative sign, just like the $u$ and $d$ quark Boer-Mulders functions of the nucleons. Using the bag model, the valence Boer-Mulders functions for mesons and nucleons were predicted [268] to have similar magnitude with the same signs. This prediction of a universal behavior of the Boer-Mulders functions for pions and nucleons awaits experimental confirmation.

For nucleon's antiquark Boer-Mulders functions there exists only one model calculation so far. It was pointed out [269] that the meson cloud could contribute to nucleon's sea-quark Boer-Mulders functions. As discussed in Section 3, the meson cloud as an important source for sea quarks in the nucleons was evidenced by the large $\bar{d} / \bar{u}$ flavor asymmetry observed in DIS and Drell-Yan experiments. A significant fraction of the nucleon's antiquark sea comes from the meson cloud. This suggests that pion cloud can contribute to the nucleon's antiquark Boer-Mulders functions [269]. An interesting implication is that nucleon's antiquark Boer-Mulders functions would have negative signs, just like Boer-Mulders functions for pion's valence quarks.

The gauge-link operator leads to a remarkable prediction [246] that the T-odd Sivers and BoerMulders functions are process dependent, namely, they must have opposite signs depending on whether they are involved in the spacelike SIDIS or the timelike Drell-Yan process. An experimental verification of the sign-reversal prediction of the Sivers and Boer-Mulders functions would provide an important test of QCD at the confinement scale, and represents a significant step towards understanding the properties of these novel TMDs.

The Boer-Mulders functions can be extracted from the azimuthal angular distribution of hadrons produced in unpolarized SIDIS. At leading twist, the $\cos 2 \phi$ term is proportional to the product of the Boer-Mulders function $h_{1}^{\perp}$ and the Collins fragmentation function $H_{1}^{\perp}$. The azimuthal angle $\phi$ refers to the angle between the hadron plane and the lepton plane. At the low $p_{T}$ region, the $\langle\cos 2 \phi\rangle$ moment has been measured by the HERMES [270, 271] and COMPASS [272, 273] Collaborations. An analysis of these $\langle\cos 2 \phi\rangle$ data for pion SIDIS, taking into account the higher-twist Cahn effect [274], was performed [275] by assuming the functional form for the Boer-Mulders function as

$$
h_{1}^{\perp q}\left(x, k_{\perp}^{2}\right)=\lambda_{q} f_{1 T}^{\perp q}\left(x, k_{\perp}^{2}\right),
$$

where $q$ refers to the quark flavor and $h_{1}^{\perp q}$ and $f_{1 T}^{\perp q}$ are the Boer-Mulders and Sivers functions, respectively. Equation 57 assumes the same $x$ and $k_{\perp}^{2}$ dependence for the Boer-Mulders and Sivers functions with the proportionality factor $\lambda_{q}$ determined by data. The pion SIDIS data are not yet able to constrain the antiquark Boer-Mulders functions, which are expected to be dominated by their quark counterparts. Therefore, the antiquark Boer-Mulders functions were assumed to be equal in magnitude to the corresponding Sivers function with a negative sign, namely,

$$
h_{1}^{\perp \bar{q}}\left(x, k_{\perp}^{2}\right)=-\left|f_{1 T}^{\perp \bar{q}}\left(x, k_{\perp}^{2}\right)\right| .
$$

The Sivers functions determined from a fit [255] to the polarized SIDIS data together with the Collins function from Ref. [276] were used. The best-fit values are $\lambda_{u}=2.0 \pm 0.1$ and $\lambda_{d}=-1.111 \pm 0.001$. Since the Sivers function for $u(d)$ is negative (positive), these values imply that $h_{1}^{\perp u}$ and $h_{1}^{\perp d}$ are both negative 
in agreement with the theoretical expectation. It should be cautioned that the extracted signs of the Boer-Mulders functions depend on the signs of the Collins functions adopted in the analysis. Although the signs chosen for the Collins functions are based on plausible arguments, some uncertainties do remain in determining the signs of the Boer-Mulders functions.

The HERMES collaboration recently reported [271] results on the azimuthal $\cos 2 \phi$ modulations for $\pi^{ \pm}, K^{ \pm}$, and unidentified hadrons in unpolarized $e+p$ and $e+d$ SIDIS. The $K^{ \pm}$and unidentified hadron data were not included in the earlier work [275] to extract nucleon Boer-Mulders functions. These new HERMES data could lead to a more precise extraction of the valence Boer-Mulders functions. In addition, these data are sensitive to the sea-quark Boer-Mulders functions. In particular, the $\cos 2 \phi$ moments for $K^{-}$production are observed to be large and negative [271], as shown in Fig. 32. Since the valence quark content of $K^{-}, s \bar{u}$, is distinct from that of the target nucleons, the large negative $K^{-} \cos 2 \phi$ moments suggest sizable sea-quark Boer-Mulders functions. An extension of the global fit in Ref. [275] to include the new $K^{ \pm}$data would be very valuable and could determine the sea-quark Boer-Mulders functions in SIDIS.

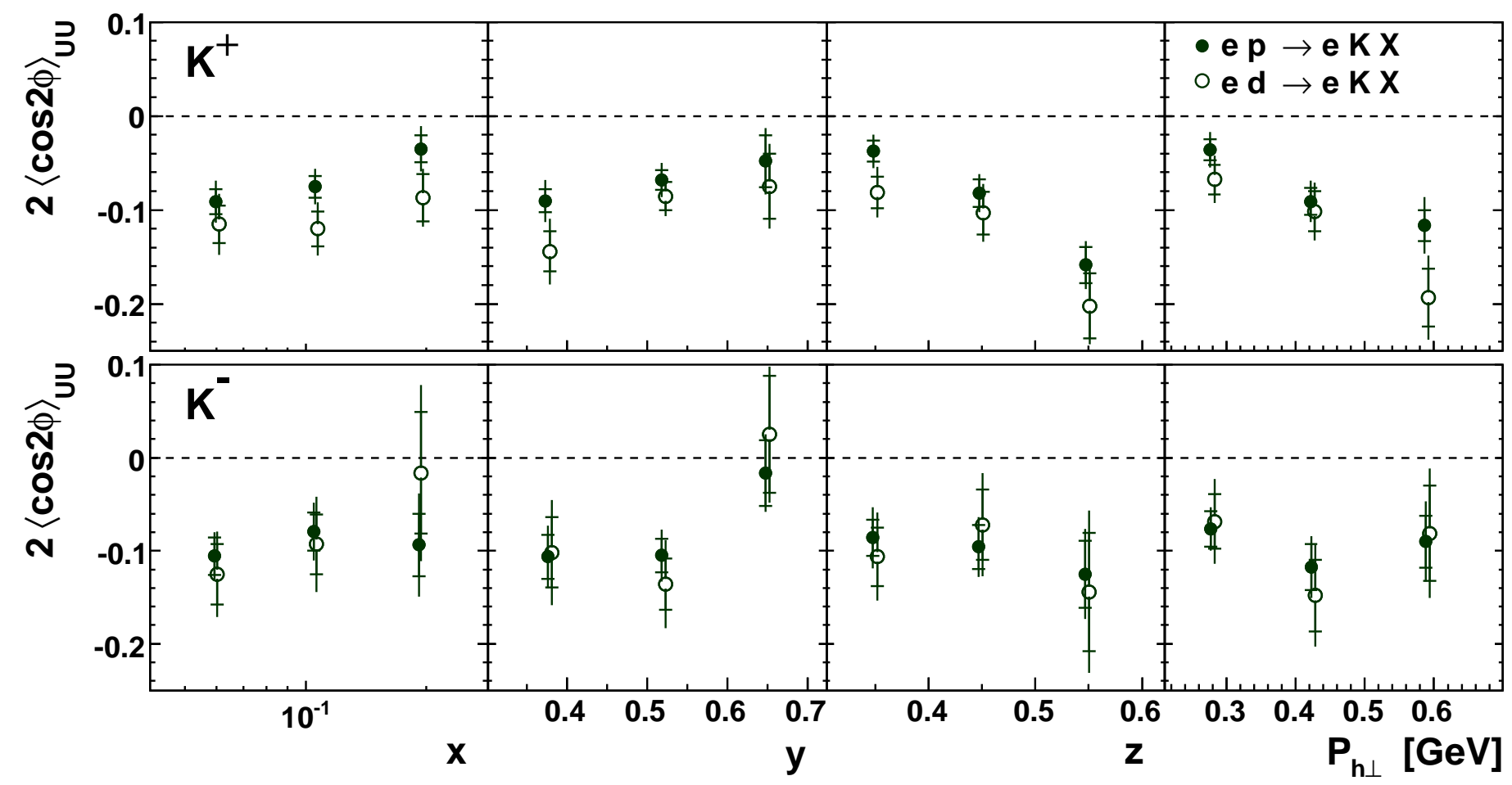

Figure 32: $\cos 2 \phi$ amplitudes for $K^{+}$and $K^{-}$in unpolarized SIDIS measurement by the HERMES Collaboration [271].

The Boer-Mulders functions can be extracted [277] from the azimuthal angular distributions in the unpolarized Drell-Yan process, $h_{1} h_{2} \rightarrow l^{+} l^{-} x$. The general expression for the Drell-Yan angular distribution is 278 ]

$$
\frac{d \sigma}{d \Omega} \propto 1+\lambda \cos ^{2} \theta+\mu \sin 2 \theta \cos \phi+\frac{\nu}{2} \sin ^{2} \theta \cos 2 \phi,
$$

where $\theta$ and $\phi$ are the polar and azimuthal decay angle of the $l^{+}$in the dilepton rest frame. Boer showed that the $\cos 2 \phi$ term is proportional to the convolution of the quark and antiquark Boer-Mulders functions in the projectile and target [277]. This can be understood by noting that the Drell-Yan cross section depends on the transverse spins of the annihilating quark and antiquark. Therefore, a correlation between the transverse spin and the transverse momentum of the quark, as represented by the BoerMulders function, would lead to a preferred transverse momentum direction. 


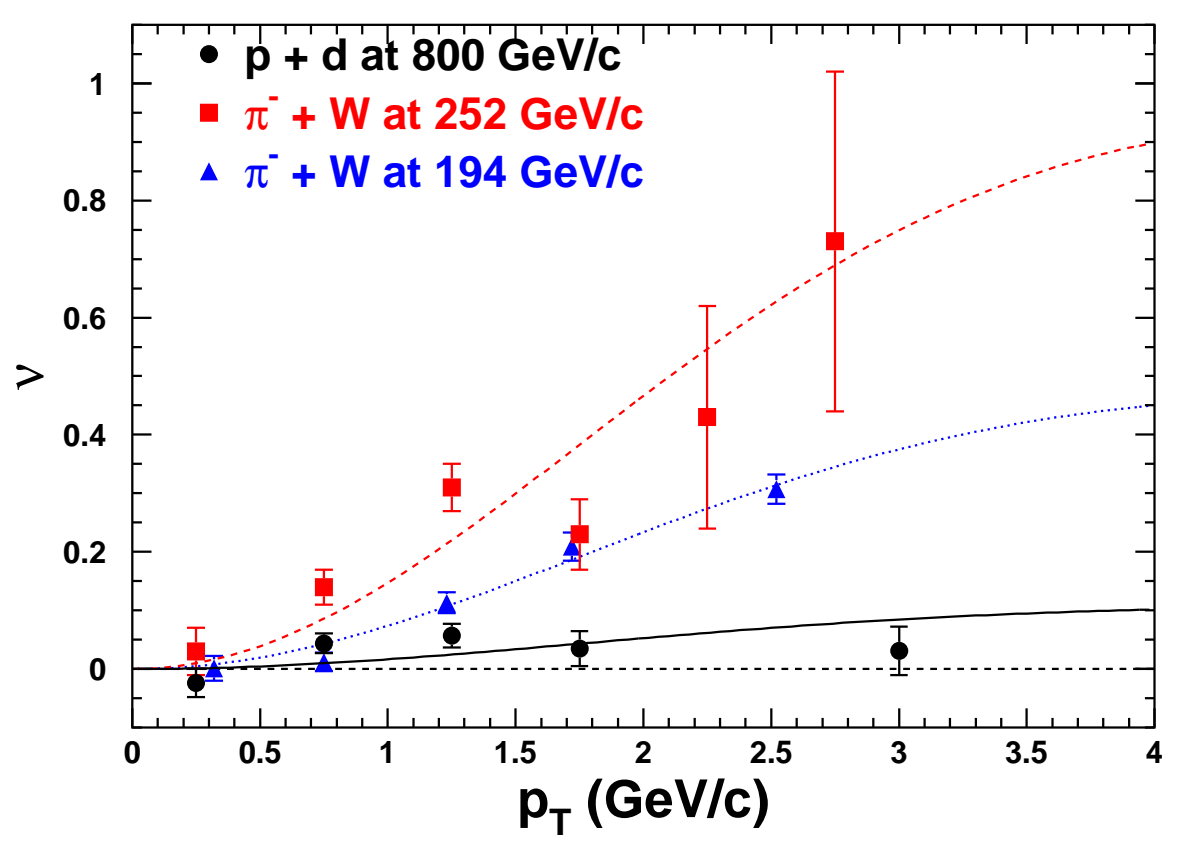

Figure 33: The $\cos 2 \phi$ amplitude $\nu$ for pion- and proton-induced Drell-Yan measurements. Curves are fits to the data using an empirical parametrization discussed in Ref. [281].

Pronounced $\cos 2 \phi$ dependence were indeed observed in the NA10 [279] and E615 [280] pion-induced Drell-Yan experiments, and attributed to the Boer-Mulders function. The first measurement of the $\cos 2 \phi$ dependence of the proton-induced Drell-Yan process was reported for $p+d$ and $p+p$ interactions at $800 \mathrm{GeV} / \mathrm{c}$ [281, 282]. In contrast to pion-induced Drell-Yan, significantly smaller (but non-zero) $\cos 2 \phi$ azimuthal angular dependence was observed in the $p+d$ and $p+p$ reactions, as shown in Fig. 33 . While the pion-induced Drell-Yan process is dominated by annihilation between a valence antiquark in the pion and a valence quark in the nucleon, the proton-induced Drell-Yan process involves a valence quark in the proton annihilating with a sea antiquark in the nucleon. Therefore, the $p+d$ and $p+p$ results suggest [281, 282] that the Boer-Mulders functions for sea antiquarks are significantly smaller than those for valence quarks.

Extractions of the Boer-Mulders functions from the $p+p$ and $p+d$ Drell-Yan data have been carried out by several groups [283, 284, 285]. Figure 34 shows the fits obtained by Lu and Schmidt [284]. The statistical accuracy of the data does not yet allow a precise extraction of Boer-Mulders functions. Nevertheless, the analysis suggests that both the $\bar{u}$ and $\bar{d}$ Boer-Mulders functions can be extracted from the Drell-Yan. Ongoing and future unpolarized pion- and proton-induced Drell-Yan experiments are expected to provide new information on the Boer-Mulders functions.

\section{Experimental Perspectives}

In this section we outline the current experimental programs and the future prospects which are related to the study of flavor structure of nucleon sea. These activities are to be carried out by fixed-target and collider experiments in various laboratories worldwide: Fermilab, CERN, BNL, JLab, FAIR, NICA, and J-PARC. 

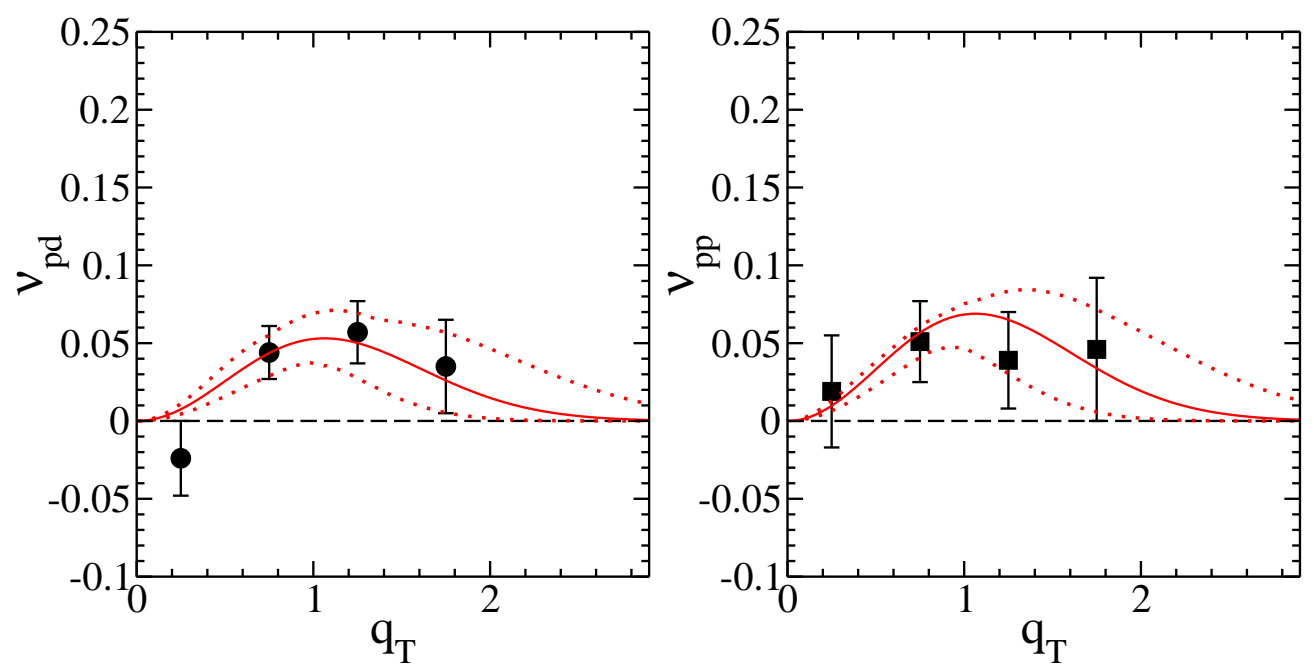

Figure 34: Fits to the $\cos 2 \phi$ amplitude $\nu$ for the $p+d$ and $p+p$ Drell-Yan data [284]. The dotted lines indicate the uncertainty of the fitted parameters. $q_{T}$ in the unit of $\mathrm{GeV}$ refers to the transverse momentum of the dimuon pair.

\subsection{Fixed-Target Experiments}
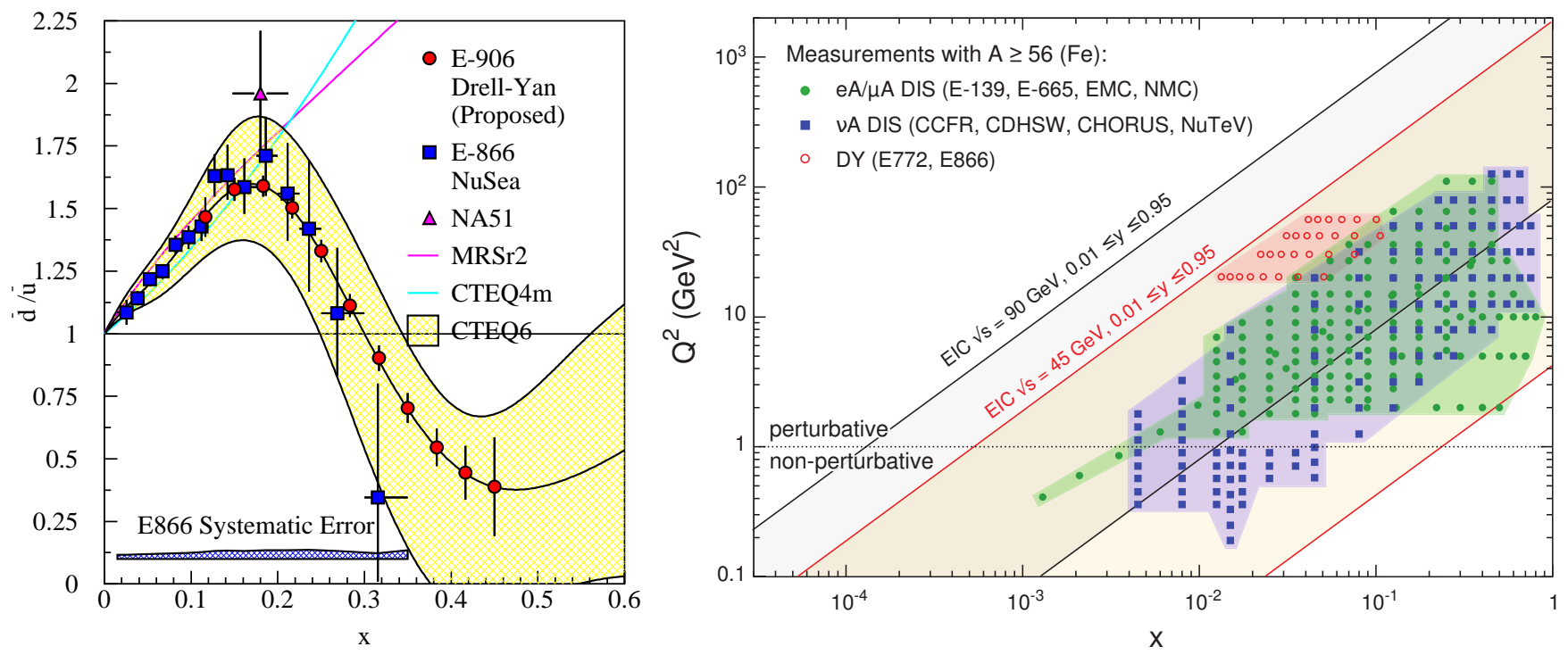

Figure 35: Left (a): Expected sensitivity of $\bar{d} / \bar{u}$ as a function of $x$ for the Fermilab E906/SeaQuest experiment [287]. Right (b): The kinematic acceptance in $x$ and $Q^{2}$ of completed lepton-nucleus DIS and fixed-target Drell-Yan experiments compared with EIC. Figure from [296].

While many theoretical models can describe the enhancement of $\bar{d}$ over $\bar{u}$, none of them predict that the $\bar{d} / \bar{u}$ ratio falls below unity which is tentatively suggested by the E866 Drell-Yan data at large $x(x>0.2)$ and the NMC data [55]. If confirmed by more precise measurements, this intriguing $x$ dependence of the $\bar{d} / \bar{u}$ asymmetry could shed important new light on the nature of the nucleon sea. Thus, it would be very important to have new measurements sensitive to the $\bar{d} / \bar{u}$ ratios at large $x(x>0.2)$. The on-going Fermilab E906/SeaQuest Drell-Yan experiment [286, 287] utilizing 120 GeV proton beam will hopefully extend the measurement of the $\bar{d} / \bar{u}$ over the region $0.25<x<0.5$ in the near future, as shown in Fig. 35(a). Furthermore there is an approved proposal of measuring the Sivers functions of 
nucleon sea quarks using transversely polarized $\mathrm{NH}_{3}$ target and the existing E906 dimuon spectrometer at Fermilab [288].

The new $50 \mathrm{GeV}$ proton accelerator, J-PARC, presents opportunities for extending the $\bar{d} / \bar{u}$ measurement to even larger $x(0.25<x<0.7)$ [289]. Since only $30 \mathrm{GeV}$ proton beam is available at the initial phase of J-PARC, the first measurements would focus on $J / \Psi$ production at $30 \mathrm{GeV}$. An important feature of $J / \Psi$ production using 30 or $50 \mathrm{GeV}$ proton beam is the dominance of the quark-antiquark annihilation subprocess. This is in striking contrast to $J / \Psi$ production at $800 \mathrm{GeV}$ (Fermilab E866) or at $120 \mathrm{GeV}$ (Fermilab E906), where the gluon-gluon fusion is the dominant process. This suggests an exciting opportunity to use $J / \Psi$ production at J-PARC as an alternative method to probe antiquark distribution.

With the capability of beam-particle identification, the usage of secondary kaon beam in the DrellYan program of COMPASS-II experiment at CERN [290, 291] and high-momentum beam line in the Hadron Hall at J-PARC [292] will offer a clean and important way of determining the strange sea.

There is a proposal of performing fixed-target experiments at LHC using the $7 \mathrm{TeV}$ proton beams [293]. With the measurement of Drell-Yan production off nucleons at the very backward region, it will measure the sea quark distributions of beam proton down to $x \approx 10^{-3}$. The measurement of open-charm or hidden-charm hadrons at large rapidities will be important for the search of the intrinsic charm component.

\subsection{Collider Experiments}

The production of $W / Z$ bosons is known to provide strong constraints on the light $u$ and $d$ quarks, both valence and sea. With an increase of collision energy, the contributions of strange and charm quark become much enhanced and the rapidity distribution of the $W / Z$ bosons provides a measure of the mix of valence and sea quarks [76]. Therefore, precision measurements of $W / Z$ production at the LHC may provide input to the global PDF fit to constrain the strange and charm in the small $x$ region. In addition, there are recent works showing the sensitivity to strange quark via the charm production in association with an $W / Z$ [78] and also to charm quark via the direct photon production in association with a charm jet 294.

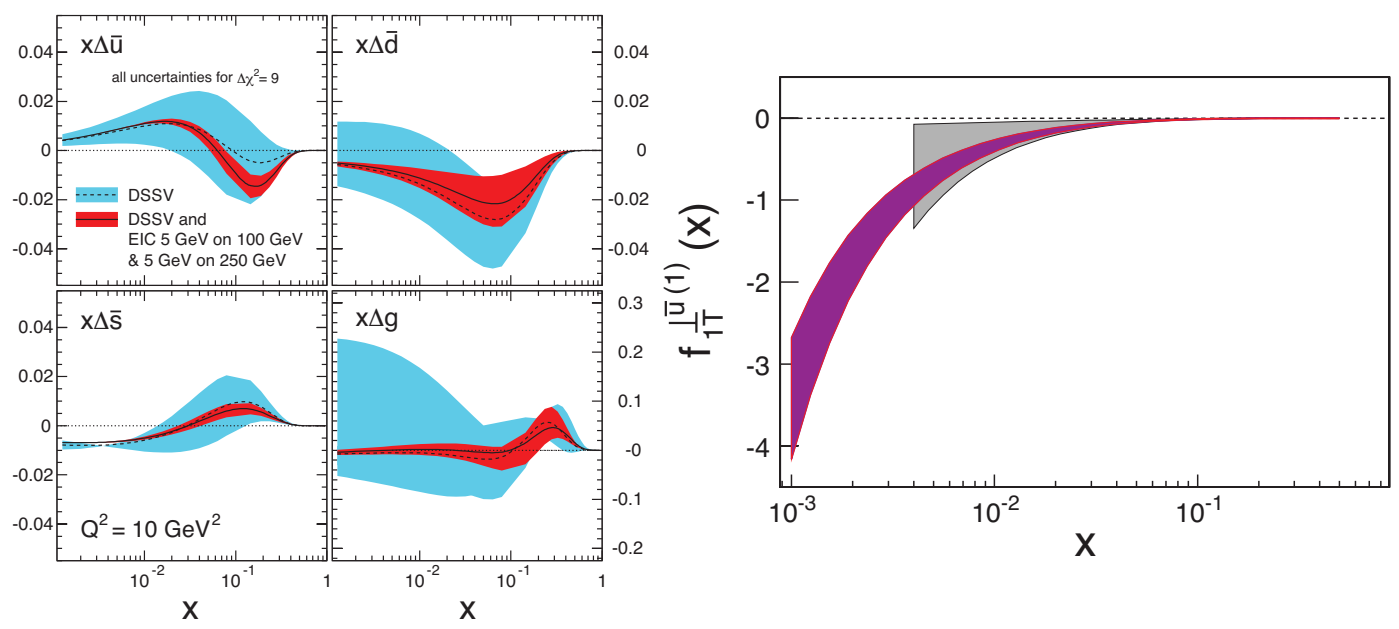

Figure 36: Left (a): Uncertainty bands on helicity parton distributions, presently (light bands) and with EIC data (darker bands), using projected inclusive and semi-inclusive EIC data sets. Right (b): Comparison of the $2 \sigma$ uncertainty of extracted Sivers function of $\bar{u}$ from currently available data (gray band) and from pseudo-data generated for the EIC with $\sqrt{s}=45 \mathrm{GeV}$ and an integrated luminosity of $10 \mathrm{fb}^{-1}$ (purple band). Figures from [296]. 
There is a proposal of constructing an electron ion collider (EIC) with either the CEBAF accelerator at JLab or the RHIC collider at BNL [295, 296]. This facility is aimed at addressing how the sea quarks and gluons, and their spins, are distributed in spatial and momentum space inside the nucleon with polarized ep and eA collisions at $\sqrt{s}=45-90 \mathrm{GeV}$. As shown in Figs. 35(b) and 36, the availability of this facility will greatly expand the explored kinematic regions of nucleon sea quarks in term of distributions of partonic density, helicity density and TMDs, especially at small- $x$ regimes.

\section{Summary}

The nucleon sea is an indispensable part of the nucleon which is a system bound by the strong interaction. As discussed in this review, studies of the nucleon sea have revealed a rich flavor structure beyond early simple pictures of gluon splitting and an SU(3) symmetric sea. In conclusion, let us summarize what are learned so far about the flavor structure of the nucleon sea.

- The observations of the Gottfried sum rule violation in a DIS experiment by NMC and the measurements of $\bar{d}(x) / \bar{u}(x)$ in Drell-Yan experiments by NA51 and E866 and SIDIS experiment by HERMES clearly establish the fact that the unpolarized distributions of $\bar{u}$ and $\bar{d}$ in the proton are strikingly different. At the region of $x>0.3$, the E866 data suggests that the $\bar{d} / \bar{u}$ falls below unity, albeit with large experimental uncertainty.

- The non-perturbative models of a cloud of virtual mesons surrounding the nucleon provide quantitative descriptions of the observed $\bar{d}(x) / \bar{u}(x)$. None of them predicts that $\bar{d} / \bar{u}$ falls below unity at any value of $x$. New attempts of statistical and balance models based on the statistical properties of partons bound inside the nucleon could also reasonably describe the flavor structures of $\bar{u}$ and $\bar{d}$. This flavor structure has been recently interpreted as the evidence of either intrinsic light sea abundant at valence region or sea quarks originated from the connected diagrams.

- As for the quark sea with strange or charm flavors, fewer data are available. The momentum fraction carried by the strange sea is measured to be about half of that carried by $\bar{u}$ and $\bar{d}$ at $Q^{2}=1 \mathrm{GeV}^{2}$ in the neutrino DIS experiment by CCFR. This suggests the breaking of $\mathrm{SU}(3)$ flavor symmetric sea. Nevertheless the recent results of $s(x)+\bar{s}(x)$ from HERMES and ATLAS experiments suggest an SU(3) flavor symmetric quarks sea at $x \sim 0.02$. The possible difference between $s(x)$ and $\bar{s}(x)$ was not excluded by the measurement of CCFR and NuTeV experiments. The charm production at the high $x$ and $Q^{2}$ regions in the DIS experiments by EMC shows possible evidences of a nonperturbative intrinsic charm component of the nucleon sea. However the most advanced global analysis shows that the currently available data could not effectively constrain the existence of this component and more precise experimental measurements are needed.

- Experimental data from polarized DIS, polarized SIDIS, and the single-spin asymmetry of $W$ boson production in $p p$ collision show that sea quarks in the nucleons have small, but nonzero polarizations. The SU(2) flavor asymmetry observed for the unpolarized $\bar{u}$ and $\bar{d}$ sea is also found for the helicity distributions, as the data strongly favor $\Delta \bar{u}>\Delta \bar{d}$. For the strange quarks, the polarized DIS and neutrino-nucleon elastic scattering data favor a negative polarization, while the polarized SIDIS data suggest a positive strange quark polarization, at least in the measured $x$ region. Uncertainties in the kaon fragmentation functions remain an obstacle in extracting the strange quark polarization in polarized SIDIS reaction.

- Impressive recent progress has been made in the study of the novel transverse-momentum and transverse-spin dependent parton distributions. First information on the transversity, Sivers functions, and Boer-Mulders functions has been successfully obtained in SIDIS and Drell-Yan experiments. Currently, the focus of the TMD study is primarily on the valence quarks, since the sea 
quarks are expected to have small contributions. Nevertheless, indications for non-zero sea quark Sivers and Boer-Mulders functions are already found in polarized and unpolarized SIDIS and Drell-Yan experiments. A global effort to check the QCD prediction of the sign-reversal for the T-odd Sivers and Boer-Mulders functions between the SIDIS and Drell-Yan would very likely also lead to a better understanding of the roles of sea quarks in the TMDs. The tantalizing connection between the sea-quark TMDs and the nucleon's orbital angular momentum remains to be studied.

Major surprises were found in recent decades regarding the flavor and spin structures of the nucleon sea. They have provided major challenges as well as important clues for understanding the internal structure of the nucleons. The flavor and spin structures of the nucleon sea will continue to offer important new insights on how QCD works in the nonperturbative regime.

\section{References}

[1] J. I. Friedman, Rev. Mod. Phys. 63 (1991) 615.

[2] A. De Roeck and R. S. Thorne, Prog. Part. Nucl. Phys. 66 (2011) 727 [arXiv:1103.0555 [hep-ph]].

[3] E. Perez and E. Rizvi, Rep. Prog. Phys. 76 (2013) 046201 [arXiv:1208.1178 [hep-ex]].

[4] S. Forte and G. Watt, Ann. Rev. Nucl. Part. Sci. 63 (2013) 291 [arXiv:1301.6754 [hep-ph]].

[5] P. Jimenez-Delgado, W. Melnitchouk and J. F. Owens, J. Phys. G 40 (2013) 093102 [arXiv:1306.6515 [hep-ph]].

[6] B. Lampe and E. Reya, Phys. Rept. 332 (2000) 1 [hep-ph/9810270].

[7] D. de Florian, R. Sassot, M. Stratmann and W. Vogelsang, Prog. Part. Nucl. Phys. 67 (2012) 251 [arXiv:1112.0904 [hep-ph]].

[8] C. A. Aidala, S. D. Bass, D. Hasch and G. K. Mallot, Rev. Mod. Phys. 85 (2013) 655 [arXiv:1209.2803 [hep-ph]].

[9] J. Blumlein, Prog. Part. Nucl. Phys. 69 (2013) 28 [arXiv:1208.6087 [hep-ph]].

[10] S. D. Drell and T. -M. Yan, Phys. Rev. Lett. 25 (1970) 316 [Erratum-ibid. 25 (1970) 902].

[11] W. -C. Chang and D. Dutta, Int. J. Mod. Phys. E 22 (2013) 1330020 [arXiv:1306.3971 [nucl-th]].

[12] J. -C. Peng and J. -W. Qiu, Prog. Part. Nucl. Phys. 76 (2014) 43 [arXiv:1401.0934 [hep-ph]].

[13] A. M. Cooper-Sarkar, R. C. E. Devenish and A. De Roeck, Int. J. Mod. Phys. A 13 (1998) 3385 [hep-ph/9712301].

[14] G. Sterman et al. [CTEQ Collaboration], Rev. Mod. Phys. 67 (1995) 157.

[15] H. Georgi and H. D. Politzer, Phys. Rev. D 14 (1976) 1829.

[16] J. Gao, M. Guzzi, J. Huston, H. -L. Lai, Z. Li, P. Nadolsky, J. Pumplin and D. Stump et al., Phys. Rev. D 89 (2014) 033009 [arXiv:1302.6246 [hep-ph]]; LHAPDF PDF set: http://www.hepforge. org/archive/lhapdf/pdfsets/current/CT10nnlo_as_0120.LHgrid.

[17] A. D. Martin, W. J. Stirling, R. S. Thorne and G. Watt, Eur. Phys. J. C 63 (2009) 189 [arXiv:0901.0002 [hep-ph]]; LHAPDF PDF set: http://www.hepforge.org/archive/lhapdf/ pdfsets/current/MSTW2008nnlo90cl_nf3.LHgrid.

[18] R. D. Ball, V. Bertone, S. Carrazza, C. S. Deans, L. Del Debbio, S. Forte, A. Guffanti and N. P. Hartland et al., Nucl. Phys. B 867 (2013) 244 [arXiv:1207.1303 [hep-ph]]; LHAPDF PDF set: http://www.hepforge.org/archive/lhapdf/pdfsets/current/NNPDF23_nnlo_as_ 0120. LHgrid.

[19] P. Amaudruz et al. [New Muon Collaboration], Phys. Rev. Lett. 66 (1991) 2712.

[20] M. Arneodo et al. [New Muon Collaboration], Phys. Rev. D 50 (1994) 1. 
[21] G. Preparata, P. G. Ratcliffe and J. Soffer, Phys. Rev. Lett. 66 (1991) 687.

[22] A. Baldit et al. [NA51 Collaboration], Phys. Lett. B 332 (1994) 244.

[23] E. A. Hawker et al. [NuSea Collaboration], Phys. Rev. Lett. 80 (1998) 3715 [hep-ex/9803011];

J. C. Peng et al. [NuSea Collaboration], Phys. Rev. D 58 (1998) 092004 [hep-ph/9804288];

R. S. Towell et al. [NuSea Collaboration], Phys. Rev. D 64 (2001) 052002[hep-ex/0103030].

[24] K. Ackerstaff et al. [HERMES Collaboration], Phys. Rev. Lett. 81 (1998) 5519 [hep-ex/9807013].

[25] R. D. Field and R. P. Feynman, Phys. Rev. D 15 (1977) 2590.

[26] D. A. Ross and C. T. Sachrajda, Nucl. Phys. B 149 (1979) 497.

[27] J. Speth and A. W. Thomas, Adv. Nucl. Phys. 24 (1997) 83.

[28] S. Kumano, Phys. Rept. 303 (1998) 183 [hep-ph/9702367].

[29] R. Vogt, Prog. Part. Nucl. Phys. 45 (2000) S105 [hep-ph/0011298].

[30] G. T. Garvey and J. -C. Peng, Prog. Part. Nucl. Phys. 47 (2001) 203 [nucl-ex/0109010].

[31] A. W. Thomas, Phys. Lett. B 126 (1983) 97.

[32] J. Speth and A. W. Thomas, Adv. Nucl. Phys. 24 (1997) 83.

[33] A. Szczurek, A. J. Buchmann and A. Faessler, J. Phys. G 22 (1996) 1741 [nucl-th/9609042].

[34] J. Alwall and G. Ingelman, Phys. Rev. D 71 (2005) 094015 [hep-ph/0503099].

[35] Y. Ding, R. -G. Xu and B. -Q. Ma, Phys. Lett. B 607 (2005) 101 [hep-ph/0408292].

[36] Y. Ding, R. -G. Xu and B. -Q. Ma, Phys. Rev. D 71 (2005) 094014 [hep-ph/0505153].

[37] H. Song, X. Zhang and B. -Q. Ma, Eur. Phys. J. C 71 (2011) 1542 [arXiv:1101.3378 [hep-ph]].

[38] M. Wakamatsu, Phys. Rev. D 67 (2003) 034005 [hep-ph/0209011]; Phys. Rev. D 67 (2003) 034006 [hep-ph/0212356].

[39] M. Wakamatsu, Phys. Rev. D 71 (2005) 057504 [hep-ph/0411203].

[40] M. Wakamatsu, Phys. Rev. D 79 (2009) 094028 [arXiv:0903.1886 [hep-ph]].

[41] C. Bourrely, J. Soffer and F. Buccella, Eur. Phys. J. C 23 (2002) 487 [hep-ph/0109160].

[42] C. Bourrely, F. Buccella and J. Soffer, Mod. Phys. Lett. A 18 (2003) 771 [hep-ph/0211389].

[43] C. R. V. Bourrely, J. Soffer and F. Buccella, Eur. Phys. J. C 41 (2005) 327 [hep-ph/0502180].

[44] C. Bourrely, J. Soffer and F. Buccella, Phys. Lett. B 648 (2007) 39 [hep-ph/0702221].

[45] Y. -h. Zhang, L. Shao and B. -Q. Ma, Phys. Lett. B 671 (2009) 30 [arXiv:0812.3294 [hep-ph]].

[46] Y. -j. Zhang, B. Zhang and B. -Q. Ma, Phys. Lett. B 523 (2001) 260 [hep-ph/0106074].

[47] Y. -J. Zhang, B. -S. Zou and L. -M. Yang, Phys. Lett. B 528 (2002) 228 [hep-ph/0201214].

[48] Y. -J. Zhang, W. -Z. Deng and B. -Q. Ma, Phys. Rev. D 65 (2002) 114005 [hep-ph/0204344].

[49] M. A. Alberg, Prog. Part. Nucl. Phys. 61 (2008) 140.

[50] B. Zhang and Y. -J. Zhang, Phys. Rev. D 82 (2010) 074021 [arXiv:1008.0467 [hep-ph]].

[51] A. W. Thomas, W. Melnitchouk and F. M. Steffens, Phys. Rev. Lett. 85 (2000) 2892 [hep$\mathrm{ph} / 0005043]$.

[52] H. -W. Lin, J. -W. Chen, S. D. Cohen and X. Ji, arXiv:1402.1462 [hep-ph].

[53] X. Ji, Phys. Rev. Lett. 11026 (2013) 262002 [arXiv:1305.1539 [hep-ph]].

[54] J. F. Owens, A. Accardi and W. Melnitchouk, Phys. Rev. D 87 (2013) 094012 [arXiv:1212.1702 [hep-ph]].

[55] J. -C. Peng, W. -C. Chang, H. -Y. Cheng, T. -J. Hou, K. -F. Liu and J. -W. Qiu, Phys. Lett. B 736 (2014) 411 [arXiv:1401.1705 [hep-ph]].

[56] P. Jimenez-Delgado and E. Reya, Phys. Rev. D 89 (2014) 074049 [arXiv:1403.1852 [hep-ph]]. 
[57] M. Arneodo et al. [New Muon. Collaboration], Phys. Lett. B 364 (1995) 107 [hep-ph/9509406].

[58] M. Arneodo et al. [New Muon Collaboration], Nucl. Phys. B 487 (1997) 3 [hep-ex/9611022].

[59] A. Accardi, W. Melnitchouk, J. F. Owens, M. E. Christy, C. E. Keppel, L. Zhu and J. G. Morfin, Phys. Rev. D 84 (2011) 014008 [arXiv:1102.3686 [hep-ph]].

[60] W. M. Alberico, S. M. Bilenky and C. Maieron, Phys. Rept. 358 (2002) 227 [hep-ph/0102269].

[61] U. Dore, Eur. Phys. J. H 37 (2012) 115 [arXiv:1103.4572 [hep-ex]].

[62] H. Abramowicz, J. G. H. de Groot, J. Knobloch, J. May, P. Palazzi, A. Para, F. Ranjard and J. Rothberg et al., Z. Phys. C 15 (1982) 19.

[63] S. A. Rabinowitz, C. Arroyo, K. T. Bachmann, A. O. Bazarko, T. Bolton, C. Foudas, B. J. King and W. Lefmann et al., Phys. Rev. Lett. 70 (1993) 134.

[64] A. O. Bazarko et al. [CCFR Collaboration], Z. Phys. C 65 (1995) 189 [hep-ex/9406007].

[65] P. Vilain et al. [CHARM II Collaboration], Eur. Phys. J. C 11 (1999) 19.

[66] P. Astier et al. [NOMAD Collaboration], Phys. Lett. B 486 (2000) 35.

[67] O. Samoylov et al. [NOMAD Collaboration], Nucl. Phys. B 876 (2013) 339 [arXiv:1308.4750 [hep$\mathrm{ex}]$.

[68] M. Goncharov et al. [NuTeV Collaboration], Phys. Rev. D 64 (2001) 112006 [hep-ex/0102049].

[69] G. P. Zeller et al. [NuTeV Collaboration], Phys. Rev. Lett. 88 (2002) 091802 [Erratum-ibid. 90 (2003) 239902] [hep-ex/0110059].

[70] G. P. Zeller et al. [NuTeV Collaboration], Phys. Rev. D 65 (2002) 111103 [Erratum-ibid. D 67 (2003) 119902] [hep-ex/0203004].

[71] D. Mason et al. [NuTeV Collaboration], Phys. Rev. Lett. 99 (2007) 192001.

[72] A. Kayis-Topaksu et al. [CHORUS Collaboration], Nucl. Phys. B 798 (2008) 1 [arXiv:0804.1869 [hep-ex]].

[73] A. Kayis-Topaksu et al. [CHORUS Collaboration], New J. Phys. 13 (2011) 093002 [arXiv:1107.0613 [hep-ex]].

[74] A. Airapetian et al. [HERMES Collaboration], Phys. Lett. B 666 (2008) 446 [arXiv:0803.2993 [hep-ex]].

[75] A. Airapetian et al. [HERMES Collaboration], Phys. Rev. D 89 (2014) 097101 [arXiv:1312.7028 [hep-ex]].

[76] A. Kusina, T. Stavreva, S. Berge, F. I. Olness, I. Schienbein, K. Kovarik, T. Jezo and J. Y. Yu et al., Phys. Rev. D 85 (2012) 094028 [arXiv:1203.1290 [hep-ph]].

[77] U. Baur, F. Halzen, S. Keller, M. L. Mangano and K. Riesselmann, Phys. Lett. B 318 (1993) 544 [hep-ph/9308370].

[78] W. J. Stirling and E. Vryonidou, Phys. Rev. Lett. 109 (2012) 082002 [arXiv:1203.6781 [hep-ph]].

[79] G. Aad et al. [ATLAS Collaboration], Phys. Rev. Lett. 109 (2012) 012001 [arXiv:1203.4051 [hep$\mathrm{ex}]$ ].

[80] G. Aad et al. [ATLAS Collaboration], JHEP 1405 (2014) 068 arXiv:1402.6263 [hep-ex].

[81] F. D. Aaron et al. [H1 and ZEUS Collaboration], JHEP 1001 (2010) 109 [arXiv:0911.0884 [hep-ex]].

[82] S. Chatrchyan et al. [CMS Collaboration], JHEP 1402 (2014) 013 [arXiv:1310.1138 [hep-ex]].

[83] S. Chatrchyan et al. [CMS Collaboration], arXiv:1312.6283 [hep-ex].

[84] S. Davidson, S. Forte, P. Gambino, N. Rius and A. Strumia, JHEP 0202 (2002) 037 [hep$\mathrm{ph} / 0112302]$.

[85] F. G. Cao and A. I. Signal, Phys. Lett. B 559 (2003) 229 [hep-ph/0302206]. 
[86] S. Kretzer, F. Olness, J. Pumplin, D. Stump, W. -K. Tung and M. H. Reno, Phys. Rev. Lett. 93 (2004) 041802 [hep-ph/0312322].

[87] J. Alwall and G. Ingelman, Phys. Rev. D 70 (2004) 111505 [hep-ph/0407364].

[88] P. Gao and B. -Q. Ma, Phys. Rev. D 77 (2008) 054002 [arXiv:0802.2071 [hep-ph]].

[89] S. Moch, J. A. M. Vermaseren and A. Vogt, Nucl. Phys. B 688 (2004) 101 [hep-ph/0403192].

[90] S. Catani, D. de Florian, G. Rodrigo and W. Vogelsang, Phys. Rev. Lett. 93 (2004) 152003 [hep$\mathrm{ph} / 0404240]$.

[91] A. I. Signal and A. W. Thomas, Phys. Lett. B 191 (1987) 205.

[92] H. Holtmann, A. Szczurek and J. Speth, Nucl. Phys. A 596 (1996) 631 [hep-ph/9601388].

[93] S. J. Brodsky and B. -Q. Ma, Phys. Lett. B 381 (1996) 317 [hep-ph/9604393].

[94] F. -G. Cao and A. I. Signal, Phys. Rev. D 60 (1999) 074021 [hep-ph/9907297].

[95] G. -Q. Feng, F. -G. Cao, X. -H. Guo and A. I. Signal, Eur. Phys. J. C 72 (2012) 2250 [arXiv:1206.1688 [hep-ph]].

[96] S. Alekhin, J. Bluemlein, L. Caminadac, K. Lipka, K. Lohwasser, S. Moch, R. Petti and R. Placakyte, arXiv:1404.6469 [hep-ph].

[97] S. J. Brodsky, P. Hoyer, C. Peterson and N. Sakai, Phys. Lett. B 93 (1980) 451; S. J. Brodsky, C. Peterson and N. Sakai, Phys. Rev. D 23 (1981) 2745.

[98] P. Hoyer and S. J. Brodsky, AIP Conf. Proc. 221 (1990) 238.

[99] W. -C. Chang and J. -C. Peng, Phys. Rev. Lett. 106 (2011) 252002 [arXiv:1102.5631 [hep-ph]].

[100] W. -C. Chang and J. -C. Peng Phys. Lett. B 704 (2011) 197 [arXiv:1105.2381 [hep-ph]].

[101] J. -C. Peng and W. -C. Chang, PoS QNP 2012 (2012) 012 [arXiv:1207.2193 [hep-ph]].

[102] P. M. Nadolsky, H. -L. Lai, Q. -H. Cao, J. Huston, J. Pumplin, D. Stump, W. -K. Tung and C. -P. Yuan, Phys. Rev. D 78 (2008) 013004 [arXiv:0802.0007 [hep-ph]].

[103] C. S. An and B. Saghai, Phys. Rev. C 85 (2012) 055203 [arXiv:1204.0300 [hep-ph]].

[104] T. Doi et al. [QCD Collaboration], PoS LATTICE 2008 (2008) 163 [arXiv:0810.2482 [hep-lat]].

[105] K. -F. Liu, W. -C. Chang, H. -Y. Cheng and J. -C. Peng, Phys. Rev. Lett. 109 (2012) 252002 [arXiv:1206.4339 [hep-ph]].

[106] J. J. Aubert et al. [European Muon Collaboration], Nucl. Phys. B 213 (1983) 31.

[107] J. J. Aubert et al. [European Muon Collaboration], Phys. Lett. B 123 (1983) 275.

[108] J. J. Aubert et al. [European Muon Collaboration], Phys. Lett. B 110 (1982) 73.

[109] J. J. Aubert et al. [European Muon Collaboration], Phys. Lett. B 94 (1980) 101.

[110] J. J. Aubert et al. [European Muon Collaboration], Phys. Lett. B 94 (1980) 96.

[111] J. Pumplin, Phys. Rev. D 73 (2006) 114015 [hep-ph/0508184].

[112] T. J. Hobbs, J. T. Londergan and W. Melnitchouk, Phys. Rev. D 89 (2014) 074008 [arXiv:1311.1578 [hep-ph]].

[113] J. Pumplin, H. L. Lai and W. K. Tung, Phys. Rev. D 75 (2007) 054029 [hep-ph/0701220].

[114] S. Dulat, T. -J. Hou, J. Gao, J. Huston, J. Pumplin, C. Schmidt, D. Stump and C. -P. Yuan, Phys. Rev. D 89 (2014) 073004 [arXiv:1309.0025 [hep-ph]].

[115] F. D. Aaron et al. [H1 and ZEUS Collaboration], JHEP 1001 (2010) 109 [arXiv:0911.0884 [hep$\mathrm{ex}]$.

[116] H. Abramowicz et al. [H1 and ZEUS Collaborations], Eur. Phys. J. C 73 (2013) 2311 [arXiv:1211.1182 [hep-ex]].

[117] R. J. Fries and A. Schafer, Phys. Lett. B 443 (1998) 40 [hep-ph/9805509]. 
[118] K. G. Boreskov and A. B. Kaidalov, Eur. Phys. J. C 10 (1999) 143 [hep-ph/9809398].

[119] F. -G. Cao and A. I. Signal, Eur. Phys. J. C 21 (2001) 105 [hep-ph/0103113].

[120] S. Kumano and M. Miyama, Phys. Rev. D 65 (2002) 034012 [hep-ph/0110097].

[121] A. W. Schreiber, A. I. Signal and A. W. Thomas, Phys. Rev. D 44 (1991) 2653; F. M. Steffens and A. W. Thomas, Phys. Rev. C 55 (1997) 900 [nucl-th/9612056].

[122] A. E. Dorokhov and N. I. Kochelev, Phys. Lett. B 259 (1991) 335; Phys. Lett. B 304 (1993) 167.

[123] A. E. Dorokhov, Czech. J. Phys. 52 (2002) C79 [hep-ph/0112332].

[124] D. Diakonov, V. Petrov, P. Pobylitsa, M. V. Polyakov and C. Weiss, Nucl. Phys. B 480 (1996) 341 [hep-ph/9606314].

[125] M. Wakamatsu and T. Kubota, Phys. Rev. D 57 (1998) 5755 [hep-ph/9707500].

[126] R. S. Bhalerao, Phys. Rev. C 63 (2001) 025208 [hep-ph/0003075].

[127] E. J. Eichten, I. Hinchliffe and C. Quigg, Phys. Rev. D 45 (1992) 2269.

[128] M. Gluck, E. Reya, M. Stratmann and W. Vogelsang, Phys. Rev. D 63 (2001) 094005 [hep$\mathrm{ph} / 0011215]$.

[129] F. M. Steffens, Phys. Lett. B 541 (2002) 346 [hep-ph/0204094].

[130] B. Dressler, K. Goeke, P. V. Pobylitsa, M. V. Polyakov, T. Watabe and C. Weiss, hep-ph/9809487.

[131] M. Wakamatsu and T. Watabe, Phys. Rev. D 62 (2000) 017506 [hep-ph/9908425].

[132] R. J. Fries, A. Schafer and C. Weiss, Eur. Phys. J. A 17 (2003) 509 [hep-ph/0204060].

[133] J. Ashman et al. [European Muon Collaboration], Nucl. Phys. B 328 (1989) 1.

[134] B. Adeva et al. [Spin Muon Collaboration], Phys. Rev. D 58 (1998) 112001.

[135] P. L. Anthony et al. [E142 Collaboration], Phys. Rev. D 54 (1996) 6620 [hep-ex/9610007].

[136] K. Abe et al. [E143 Collaboration], Phys. Rev. D 58 (1998) 112003 [hep-ph/9802357].

[137] K. Abe et al. [E154 Collaboration], Phys. Rev. Lett. 79 (1997) 26 [hep-ex/9705012].

[138] P. L. Anthony et al. [E155 Collaboration], Phys. Lett. B 493 (2000) 19 [hep-ph/0007248].

[139] P. L. Anthony et al. [E155 Collaboration], Phys. Lett. B 463 (1999) 339 [hep-ex/9904002].

[140] V. Y. Alexakhin et al. [COMPASS Collaboration], Phys. Lett. B 647 (2007) 8 [hep-ex/0609038].

[141] M. G. Alekseev et al. [COMPASS Collaboration], Phys. Lett. B 690 (2010) 466 [arXiv:1001.4654 [hep-ex]].

[142] K. Ackerstaff et al. [HERMES Collaboration], Phys. Lett. B 464 (1999) 123 [hep-ex/9906035].

[143] A. Airapetian et al. [HERMES Collaboration], Phys. Rev. D 75 (2007) 012007 [hep-ex/0609039].

[144] X. Zheng et al. [Jefferson Lab Hall A Collaboration], Phys. Rev. Lett. 92 (2004) 012004 [nuclex/0308011]; Phys. Rev. C 70 (2004) 065207 [nucl-ex/0405006].

[145] K. V. Dharmawardane et al. [CLAS Collaboration], Phys. Lett. B 641 (2006) 11[nucl-ex/0605028].

[146] M. Gluck, E. Reya, M. Stratmann and W. Vogelsang, Phys. Rev. D 53 (1996) 4775 [hepph/9508347]; M. Gluck, E. Reya, M. Stratmann and W. Vogelsang, Phys. Rev. D 63 (2001) 094005 [hep-ph/0011215].

[147] J. Blumlein and H. Bottcher, Nucl. Phys. B 636 (2002) 225 [hep-ph/0203155].

[148] J. Blumlein and H. Bottcher, Nucl. Phys. B 841 (2010) 205 [arXiv:1005.3113 [hep-ph]].

[149] E. Leader, A. V. Sidorov and D. B. Stamenov, Phys. Rev. D 75 (2007) 074027 [hep-ph/0612360].

[150] E. Leader, A. V. Sidorov and D. B. Stamenov, Phys. Rev. D 82 (2010) 114018 [arXiv:1010.0574 [hep-ph]].

[151] M. Hirai, S. Kumano and N. Saito, Phys. Rev. D 74 (2006) 014015 [hep-ph/0603213]. 
[152] M. Hirai et al. [Asymmetry Analysis Collaboration], Nucl. Phys. B 813 (2009) 106 [arXiv:0808.0413 [hep-ph]].

[153] C. Bourrely, J. Soffer and F. Buccella, Eur. Phys. J. C 23 (2002) 487 [hep-ph/0109160].

[154] R. D. Ball et al. [The NNPDF Collaboration], Nucl. Phys. B 874 (2013) 36 [arXiv:1303.7236 [hep-ph]].

[155] J. M. Moss, AIP Conf. Proc. 339 (1994) 721.

[156] C. Bourrely and J. Soffer, Phys. Lett. B 314 (1993) 132.

[157] M. L. Mangano, S. I. Alekhin, M. Anselmino, R. D. Ball, M. Boglione, U. D’Alesio, S. Davidson and D. Lellis et al., CERN Yellow Report CERN-2004-002, pp.185-257 [hep-ph/0105155].

[158] D. Boer, M. Diehl, R. Milner, R. Venugopalan, W. Vogelsang, D. Kaplan, H. Montgomery and S. Vigdor et al., arXiv:1108.1713 [nucl-th].

[159] B. Adeva et al. [Spin Muon (SMC) Collaboration], Phys. Lett. B 369 (1996) 93.

[160] B. Adeva et al. [Spin Muon Collaboration], Phys. Lett. B 420 (1998) 180 [hep-ex/9711008].

[161] K. Ackerstaff et al. [HERMES Collaboration], Phys. Lett. B 464 (1999) 123 [hep-ex/9906035].

[162] A. Airapetian et al. [HERMES Collaboration], Phys. Rev. Lett. 92 (2004) 012005 [hepex/0307064].

[163] A. Airapetian et al. [HERMES Collaboration], Phys. Rev. D 71 (2005) 012003 [hep-ex/0407032].

[164] M. Alekseev et al. [COMPASS Collaboration], Phys. Lett. B 660 (2008) 458 [arXiv:0707.4077 [hep-ex]].

[165] M. Alekseev et al. [COMPASS Collaboration], Phys. Lett. B 680 (2009) 217 [arXiv:0905.2828 [hep-ex]].

[166] M. G. Alekseev et al. [COMPASS Collaboration], Phys. Lett. B 693 (2010) 227 [arXiv:1007.4061 [hep-ex]].

[167] D. de Florian, R. Sassot, M. Stratmann and W. Vogelsang, Phys. Rev. D 80 (2009) 034030 [arXiv:0904.3821 [hep-ph]].

[168] D. de Florian, R. Sassot and M. Stratmann, Phys. Rev. D 75 (2007) 114010 [hep-ph/0703242 [HEP-PH]].

[169] E. Leader, A. V. Sidorov and D. B. Stamenov, Phys. Part. Nucl. 45 (2014) 43 [arXiv:1212.3204].

[170] G. Bunce, N. Saito, J. Soffer and W. Vogelsang, Ann. Rev. Nucl. Part. Sci. 50 (2000) 525 [hep$\mathrm{ph} / 0007218]$.

[171] D. de Florian and W. Vogelsang, Phys. Rev. D 81 (2010) 094020 [arXiv:1003.4533 [hep-ph]].

[172] P. M. Nadolsky and C. P. Yuan, Nucl. Phys. B 666 (2003) 31 [hep-ph/0304002].

[173] A. Adare et al. [PHENIX Collaboration], Phys. Rev. Lett. 106 (2011) 062001 [arXiv:1009.0505 [hep-ex]].

[174] M. M. Aggarwal et al. [STAR Collaboration], Phys. Rev. Lett. 106 (2011) 062002 [arXiv:1009.0326 [hep-ex]].

[175] J. R. Stevens [ STAR Collaboration], arXiv:1302.6639 [nucl-ex].

[176] E. C. Aschenauer, A. Bazilevsky, K. Boyle, K. O. Eyser, R. Fatemi, C. Gagliardi, M. GrossePerdekamp and J. Lajoie et al., arXiv:1304.0079 [nucl-ex].

[177] Q. Xu, Proceedings of DSPIN-13 (2013).

[178] H. Oide, Phys. Part. Nucl. 45 (2014) 102.

[179] J. Ashman et al. [European Muon Collaboration], Phys. Lett. B 206 (1988) 364.

[180] R. L. Jaffe and A. Manohar, Nucl. Phys. B 337 (1990) 509. 
[181] J. R. Ellis and M. Karliner, Phys. Lett. B 341 (1995) 397 [hep-ph/9407287].

[182] M. J. Savage and J. Walden, Phys. Rev. D 55 (1997) 5376 [hep-ph/9611210].

[183] S. D. Bass and A. W. Thomas, Phys. Lett. B 684 (2010) 216 [arXiv:0912.1765 [hep-ph]].

[184] H. -W. Lin and K. Orginos, Phys. Rev. D 79 (2009) 034507 [arXiv:0712.1214 [hep-lat]].

[185] G. Erkol, M. Oka and T. T. Takahashi, Phys. Lett. B 686 (2010) 36 [arXiv:0911.2447 [hep-lat]].

[186] M. Gockeler et al. [QCDSF/UKQCD Collaboration], PoS LATTICE 2010 (2010) 163 [arXiv:1102.3407 [hep-lat]].

[187] A. Bottino, F. Donato, N. Fornengo and S. Scopel, Astropart. Phys. 18 (2002) 205 [hep$\mathrm{ph} / 0111229]$.

[188] J. R. Ellis, K. A. Olive, Y. Santoso and V. C. Spanos, Phys. Rev. D 71 (2005) 095007 [hep$\mathrm{ph} / 0502001]$.

[189] J. R. Ellis, K. A. Olive and C. Savage, Phys. Rev. D 77 (2008) 065026 [arXiv:0801.3656 [hep-ph]].

[190] F. -G. Cao and A. I. Signal, Phys. Rev. D 68 (2003) 074002 [hep-ph/0306033].

[191] E. J. Eichten, I. Hinchliffe and C. Quigg, Phys. Rev. D 45 (1992) 2269.

[192] T. P. Cheng and L. -F. Li, Phys. Rev. Lett. 74 (1995) 2872 [hep-ph/9410345].

[193] T. P. Cheng and L. -F. Li, Phys. Rev. D 57 (1998) 344 [hep-ph/9701248].

[194] S. J. Dong, J. -F. Lagae and K. F. Liu, Phys. Rev. Lett. 75 (1995) 2096 [hep-ph/9502334].

[195] S. Gusken et al. [TXL Collaboration], Phys. Rev. D 59 (1999) 114502.

[196] R. Babich, R. C. Brower, M. A. Clark, G. T. Fleming, J. C. Osborn, C. Rebbi and D. Schaich, Phys. Rev. D 85 (2012) 054510 [arXiv:1012.0562 [hep-lat]].

[197] G. S. Bali et al. [QCDSF Collaboration], Phys. Rev. Lett. 108 (2012) 222001 [arXiv:1112.3354 [hep-lat]].

[198] M. Engelhardt, Phys. Rev. D 86 (2012) 114510 [arXiv:1210.0025 [hep-lat]].

[199] A. Abdel-Rehim, C. Alexandrou, M. Constantinou, V. Drach, K. Hadjiyiannakou, K. Jansen, G. Koutsou and A. Vaquero, Phys. Rev. D 89 (2014) 034501 [arXiv:1310.6339 [hep-lat]].

[200] M. Deka, T. Doi, Y. B. Yang, B. Chakraborty, S. J. Dong, T. Draper, M. Glatzmaier and M. Gong et al., arXiv:1312.4816 [hep-lat].

[201] S. Syritsyn, arXiv:1403.4686 [hep-lat].

[202] A. Silva, H. -C. Kim, D. Urbano and K. Goeke, Phys. Rev. D 72 (2005) 094011 [hep-ph/0509281].

[203] K. Goeke, H. -C. Kim, A. Silva and D. Urbano, Eur. Phys. J. A 32 (2007) 393 [hep-ph/0608262].

[204] C. S. An, D. O. Riska and B. S. Zou, Phys. Rev. C 73 (2006) 035207 [hep-ph/0511223].

[205] D. O. Riska and B. S. Zou, Phys. Lett. B 636 (2006) 265 [nucl-th/0512102].

[206] V. E. Lyubovitskij, P. Wang, T. Gutsche and A. Faessler, Phys. Rev. C 66 (2002) 055204 [hep$\mathrm{ph} / 0207225]$.

[207] N. W. Park and H. Weigel, Nucl. Phys. A 541 (1992) 453.

[208] E. Leader, A. V. Sidorov and D. B. Stamenov, Phys. Rev. D 73 (2006) 034023 [hep-ph/0512114].

[209] E. Leader, A. V. Sidorov and D. B. Stamenov, Phys. Rev. D 84 (2011) 014002 [arXiv:1103.5979 [hep-ph]].

[210] M. Hirai, S. Kumano, T. -H. Nagai and K. Sudoh, Phys. Rev. D 75 (2007) 094009 [hep$\mathrm{ph} / 0702250]$.

[211] D. de Florian, G. A. Navarro and R. Sassot, Phys. Rev. D 71 (2005) 094018 [hep-ph/0504155].

[212] A. Airapetian et al. [HERMES Collaboration], Phys. Rev. D 87 (2013) 074029 [arXiv:1212.5407 [hep-ex]]. 
[213] L. A. Ahrens, S. H. Aronson, P. L. Connolly, B. G. Gibbard, M. J. Murtagh, S. J. Murtagh, S. Terada and D. H. White et al., Phys. Rev. D 35 (1987) 785.

[214] G. T. Garvey, W. C. Louis and D. H. White, Phys. Rev. C 48 (1993) 761.

[215] D. S. Armstrong and R. D. McKeown, Ann. Rev. Nucl. Part. Sci. 62 (2012) 337 [arXiv:1207.5238 [nucl-ex]].

[216] S. F. Pate, Phys. Rev. Lett. 92 (2004) 082002 [hep-ex/0310052].

[217] S. F. Pate, D. W. McKee and V. Papavassiliou, Phys. Rev. C 78 (2008) 015207 [arXiv:0805.2889 [hep-ex]].

[218] S. Pate and D. Trujillo, EPJ Web of Conference 66 (2014) 06018 [arXiv:1308.5694 [hep-ph]].

[219] G. Pagliaroli, C. Lujan-Peschard, M. Mitra and F. Vissani, Phys. Rev. Lett. 111 (2013) 022001 [arXiv:1210.4225 [hep-ph]].

[220] D. de Florian, M. Stratmann and W. Vogelsang, Phys. Rev. Lett. 81 (1998) 530 [hep-ph/9802432].

[221] Q. -h. Xu, Z. -t. Liang and E. Sichtermann, Phys. Rev. D 73 (2006) 077503 [hep-ph/0511061].

[222] B. I. Abelev et al. [STAR Collaboration], Phys. Rev. D 80 (2009) 111102 [arXiv:0910.1428 [hepex]].

[223] V. Barone, A. Drago and P. G. Ratcliffe, Phys. Rept. 359 (2002) 1 [hep-ph/0104283].

[224] C. Bourrely, J. Soffer and O. V. Teryaev, Phys. Lett. B 420 (1998) 375 [hep-ph/9710224].

[225] J. Soffer, Phys. Rev. Lett. 74 (1995) 1292 [hep-ph/9409254].

[226] P. Schweitzer, D. Urbano, M. V. Polyakov, C. Weiss, P. V. Pobylitsa and K. Goeke, Phys. Rev. D 64 (2001) 034013 [hep-ph/0101300].

[227] M. Wakamatsu, Phys. Lett. B 653 (2007) 398[arXiv:0705.2917 [hep-ph]].

[228] C. Bourrely, F. Buccella and J. Soffer, Mod. Phys. Lett. A 24 (2009) 1889 [arXiv:0906.1971 [hep-ph]].

[229] H. W. Lin, talk presented at the ECT Workshop on "Flavor Structure of the Nucleon Sea", 2013.

[230] J. P. Ralston and D. E. Soper, Nucl. Phys. B 152 (1979) 109.

[231] V. Barone et al. [PAX Collaboration], hep-ex/0505054.

[232] J. C. Collins, Nucl. Phys. B 396 (1993) 161 [hep-ph/9208213].

[233] D. Boer, R. Jakob and P. J. Mulders, [hep-ph/9702281].

[234] K. Abe et al. [Belle Collaboration], Phys. Rev. Lett. 96 (2006) 232002 [hep-ex/0507063]; R. Seidl et al. [Belle Collaboration], Phys. Rev. D 78 (2008) 032011 [Erratum-ibid. D 86 (2012) 039905] [arXiv:0805.2975 [hep-ex]].

[235] A. Airapetian et al. [HERMES Collaboration], Phys. Rev. Lett. 94 (2005) 012002 [hepex/0408013].

[236] A. Airapetian et al. [HERMES Collaboration], Phys. Lett. B 693 (2010) 11 [arXiv:1006.4221 [hep-ex]].

[237] E. S. Ageev et al. [COMPASS Collaboration], Nucl. Phys. B 765 (2007) 31 [hep-ex/0610068].

[238] C. Adolph et al. [COMPASS Collaboration], Phys. Lett. B 717 (2012) 376 [arXiv:1205.5121 [hep$\mathrm{ex}]$.

[239] A. Martin [COMPASS Collaboration], Phys. Part. Nucl. 45 (2014) 141 [arXiv:1303.2076 [hep-ex]].

[240] X. Qian et al. [Jefferson Lab Hall A Collaboration], Phys. Rev. Lett. 107 (2011) 072003 [arXiv:1106.0363 [nucl-ex]].

[241] Y. X. Zhao et al. [Jefferson Lab Hall A Collaboration], arXiv:1404.7204 [nucl-ex]. 
[242] M. Anselmino, M. Boglione, U. D’Alesio, S. Melis, F. Murgia and A. Prokudin, Phys. Rev. D 87 (2013) 094019 [arXiv:1303.3822 [hep-ph]].

[243] H. Gao, L. Gamberg, J. P. Chen, X. Qian, Y. Qiang, M. Huang, A. Afanasev and M. Anselmino et al., Eur. Phys. J. Plus 126 (2011) 2 [arXiv:1009.3803 [hep-ph]].

[244] D. W. Sivers, Phys. Rev. D 41 (1990) 83.

[245] S. J. Brodsky, D. S. Hwang and I. Schmidt, Phys. Lett. B 530 (2002) 99 [hep-ph/0201296].

[246] J. C. Collins, Phys. Lett. B 536 (2002) 43 [hep-ph/0204004].

[247] X. -d. Ji and F. Yuan, Phys. Lett. B 543 (2002) 66 [hep-ph/0206057].

[248] A. W. Thomas, Phys. Rev. Lett. 101 (2008) 102003 [arXiv:0803.2775 [hep-ph]].

[249] G. T. Garvey, Phys. Rev. C 81 (2010) 055212 [arXiv:1001.4547 [nucl-th]].

[250] M. Wakamatsu, Eur. Phys. J. A 44 (2010) 297 [arXiv:0908.0972 [hep-ph]].

[251] M. Diefenthaler [HERMES Collaboration], arXiv:0706.2242 [hep-ex].

[252] A. Airapetian et al. [HERMES Collaboration], Phys. Rev. Lett. 103 (2009) 152002 [arXiv:0906.3918 [hep-ex]].

[253] M. Alekseev et al. [COMPASS Collaboration], Phys. Lett. B 673 (2009) 127 [arXiv:0802.2160 [hep-ex]].

[254] M. G. Alekseev et al. [COMPASS Collaboration], Phys. Lett. B 692 (2010) 240 [arXiv:1005.5609 [hep-ex]].

[255] M. Anselmino, M. Boglione, U. D’Alesio, A. Kotzinian, S. Melis, F. Murgia, A. Prokudin and C. Turk, Eur. Phys. J. A 39 (2009) 89 [arXiv:0805.2677 [hep-ph]].

[256] P. Sun and F. Yuan, Phys. Rev. D 88 (2013) 034016 [arXiv:1304.5037 [hep-ph]].

[257] J. H. Lee et al. [BRAHMS Collaboration], arXiv:0908.4551 [hep-ex].

[258] Fermilab proposal p-1039, X. Jiang, A. Klein et al. (2013).

[259] D. Boer and P. J. Mulders, Phys. Rev. D 57 (1998) 5780 [hep-ph/9711485].

[260] D. Boer, S. J. Brodsky and D. S. Hwang, Phys. Rev. D 67 (2003) 054003 [hep-ph/0211110].

[261] L. P. Gamberg, G. R. Goldstein and K. A. Oganessyan, Phys. Rev. D 67 (2003) 071504 [hepph/0301018]; A. Bacchetta, A. Schäefer and J. -J. Yang, Phys. Lett. B 578 (2004) 109 [hep$\mathrm{ph} / 0309246]$.

[262] L. P. Gamberg, G. R. Goldstein and M. Schlegel, Phys. Rev. D 77 (2008) 094016 [arXiv:0708.0324 [hep-ph]].

[263] F. Yuan, Phys. Lett. B 575 (2003) 45 [hep-ph/0308157].

[264] P. V. Pobylitsa, hep-ph/0301236.

[265] B. Pasquini, M. Pincetti and S. Boffi, Phys. Rev. D 76 (2007) 034020 [hep-ph/0612094].

[266] M. Gockeler et al. [QCDSF and UKQCD Collaborations], Phys. Rev. Lett. 98 (2007) 222001 [hep-lat/0612032].

[267] Z. Lu and B. -Q. Ma, Phys. Lett. B 615 (2005) 200 [hep-ph/0504184].

[268] M. Burkardt and B. Hannafious, Phys. Lett. B 658 (2008) 130 [arXiv:0705.1573 [hep-ph]].

[269] Z. Lu, B. -Q. Ma and I. Schmidt, Phys. Lett. B 639 (2006) 494 [hep-ph/0702006 [HEP-PH]].

[270] F. Giordano et al. [HERMES Collaboration], AIP Conf. Proc. 1149 (2009) 423 [arXiv:0901.2438 [hep-ex]].

[271] A. Airapetian et al. [HERMES Collaboration], Phys. Rev. D 87 (2013) 012010 [arXiv:1204.4161 [hep-ex]].

[272] W. Kafer [COMPASS Collaboration], arXiv:0808.0114 [hep-ex]. 
[273] A. Bressan [COMPASS Collaboration], arXiv:0907.5511 [hep-ex].

[274] R. N. Cahn, Phys. Lett. B 78 (1978) 269.

[275] V. Barone, S. Melis and A. Prokudin, Phys. Rev. D 81 (2010) 114026 [arXiv:0912.5194 [hep-ph]].

[276] M. Anselmino, M. Boglione, U. D'Alesio, A. Kotzinian, F. Murgia, A. Prokudin and S. Melis, Nucl. Phys. Proc. Suppl. 191 (2009) 98 [arXiv:0812.4366 [hep-ph]].

[277] D. Boer, Phys. Rev. D 60 (1999) 014012 [hep-ph/9902255].

[278] C. S. Lam and W. -K. Tung, Phys. Rev. D 18 (1978) 2447.

[279] NA10 Collaboration, S. Falciano et al., Z. Phys. C 31 (1986) 513; M. Guanziroli et al., Z. Phys. C 37 (1988) 545.

[280] J. S. Conway et al. [E615 Collaboration] Phys. Rev. D 39 (1989) 92.

[281] L. Y. Zhu et al. [NuSea Collaboration], Phys. Rev. Lett. 99 (2007) 082301 [hep-ex/0609005].

[282] L. Y. Zhu et al. [NuSea Collaboration], Phys. Rev. Lett. 102 (2009) 182001 [arXiv:0811.4589 [nucl-ex]].

[283] B. Zhang, Z. Lu, B. -Q. Ma and I. Schmidt, Phys. Rev. D 77 (2008) 054011 [arXiv:0803.1692 [hep-ph]].

[284] Z. Lu and I. Schmidt, Phys. Rev. D 81 (2010) 034023 [arXiv:0912.2031 [hep-ph]].

[285] V. Barone, S. Melis and A. Prokudin, Phys. Rev. D 81 (2010) 114026 [arXiv:0912.5194 [hep-ph]].

[286] D. F. Geesaman et al. [Fermilab SeaQuest Collaboration], Fermi National Accelerator Laboratory Proposal E906 (1999).

[287] P. E. Reimer [Fermilab SeaQuest Collaboration], J. Phys. Conf. Ser. 295 (2011) 012011.

[288] A. Klein and X. Jiang et al., "Drell-Yan experiment with a polarized proton target", P-1039, 2013.

[289] J. C. Peng, G. T. Garvey, J. M. Moss, S. Sawada and J. Chiba, hep-ph/0007341.

[290] O. Denisov [COMPASS Collaboration], Mod. Phys. Lett. A 24 (2009) 3033.

[291] C. Quintans [COMPASS Collaboration], J. Phys. Conf. Ser. 295 (2011) 012163.

[292] S. Sawada, talk of "Hadron experimental hall and high-momentum beamline at J-PARC" in KEK theory center workshop on Hadron physics with high-momentum hadron beams at J-PARC in 2013.

[293] S. J. Brodsky, F. Fleuret, C. Hadjidakis and J. P. Lansberg, Phys. Rept. 522 (2013) 239 [arXiv:1202.6585 [hep-ph]].

[294] T. P. Stavreva and J. F. Owens, Phys. Rev. D 79 (2009) 054017 [arXiv:0901.3791 [hep-ph]].

[295] D. Boer, M. Diehl, R. Milner, R. Venugopalan, W. Vogelsang, D. Kaplan, H. Montgomery and S. Vigdor et al., arXiv:1108.1713 [nucl-th].

[296] A. Accardi, J. L. Albacete, M. Anselmino, N. Armesto, E. C. Aschenauer, A. Bacchetta, D. Boer and W. Brooks et al., arXiv:1212.1701 [nucl-ex]. 